\title{
AUTOMORPHISMS OF COMPLEX REFLECTION GROUPS
}

\author{
I. MARIN AND J. MICHEL
}

\begin{abstract}
Let $G \subset \mathrm{GL}\left(\mathbb{C}^{r}\right)$ be a finite complex reflection group. We show that when $G$ is irreducible, apart from the exception $G=\mathfrak{S}_{6}$, as well as for a large class of non-irreducible groups, any automorphism of $G$ is the product of a central automorphism and of an automorphism which preserves the reflections. We show further that an automorphism which preserves the reflections is the product of an element of $N_{\mathrm{GL}\left(\mathbb{C}^{r}\right)}(G)$ and of a "Galois" automorphism: we show that $\operatorname{Gal}(K / \mathbb{Q})$, where $K$ is the field of definition of $G$, injects into the group of outer automorphisms of $G$, and that this injection can be chosen such that it induces the usual Galois action on characters of $G$, apart from a few exceptional characters; further, replacing $K$ if needed by an extension of degree 2 , the injection can be lifted to $\operatorname{Aut}(G)$, and every irreducible representation admits a model which is equivariant with respect to this lifting. Along the way we show that the fundamental invariants of $G$ can be chosen rational.
\end{abstract}

\section{INTRODUCTION}

Let $G$ be a finite complex reflection group of rank $r$, that is, a finite group generated by (pseudo)reflections in a vector space $V$ of dimension $r<\infty$ over $\mathbb{C}$. We say that $G$ is irreducible when $G$ acts irreducibly on $V$. In this paper, we determine the group $\operatorname{Aut}(G)$ of automorphisms of $G$ when $G$ is irreducible, as well as for a large class of non-irreducible groups.

An automorphism $\alpha \in \operatorname{Aut}(G)$ such that $\alpha(g) \in g Z G$ for all $g \in G$, where $Z G$ denotes the center of $G$, is called central. These automorphisms form a normal subgroup of $\operatorname{Aut}(G)$, denoted $C$.

We call reflections of $G$ all the elements which act as a (pseudo-)reflection on $V$ and denote by $A$ the subgroup of automorphisms of $G$ which preserve the reflections. One of our main results is the following.

Theorem 1.1. Let $G$ be an irreducible complex reflection group different from $G(1,1,6) \simeq \mathfrak{S}_{6} ;$ then $\operatorname{Aut}(G)=C \cdot A$. If $\operatorname{dim} V>2$, then $\operatorname{Aut}(G)=C \rtimes A$.

Further, we extend this result to a large class of non-irreducible groups (see Theorem 1.11).

For $G$ of rank 2, it may happen that $C$ intersects $A$ non-trivially, and for $G$ of rank 1 we have $C=A$.

The main observation of this paper is the interpretation of $A$ as "Galois" automorphisms.

Received by the editors April 8, 2009 and, in revised form, February 1, 2010.

2010 Mathematics Subject Classification. 20F55, 20F28, $20 \mathrm{C} 15$.

I. Marin benefited from the ANR Grant ANR-09-JCJC-0102-01.

(C)2010 American Mathematical Society 
Let $\chi_{V}$ be the character of $G$ afforded by $V$. It is well known (see e.g. Bens, 7.1.1]) that the representation $V$ of $G$ can be realized over the subfield $K \subset \mathbb{C}$ generated by the values of $\chi_{V}$; the field $K$ is called the field of definition of $G$. Further, by a well-known result of Benard and Bessis (Bena, Bes] any irreducible representation of $G$ can be realized over $K$.

Let $\Gamma=\operatorname{Gal}(K / \mathbb{Q})$, and let $\gamma \in \Gamma$. If $a_{\gamma}$ is an automorphism of $G$ such that for any $g \in G$ we have $\chi_{V} \circ a_{\gamma}=\gamma\left(\chi_{V}\right)$ we say that $a_{\gamma}$ is a Galois automorphism corresponding to $\gamma$.

Theorem 1.2. Assume $G$ is irreducible. Then any faithful irreducible representation where the reflections of $G$ still act by reflections is a Galois conjugate of $V$.

As a corollary, for an irreducible group, $A$ identifies with the group of Galois automorphisms.

Note that $a_{\gamma}$ is determined by $\gamma$ up to an element of the normal subgroup $N$ formed by the Galois automorphisms corresponding to the identity element of $\Gamma$. Equivalently, $N$ is the subgroup of automorphisms induced by an element of $N_{\mathrm{GL}(V)}(G)$; they contain the inner automorphisms. We call the induced outer automorphisms diagram automorphisms (since they extend the corresponding notion for finite Coxeter groups).

What is remarkable is that any element of the Galois group can be represented by an automorphism. We denote by $\operatorname{Out}(G)$ the group of outer automorphisms, identified with $\operatorname{Aut}(G) /(G / Z G)$. Let $\bar{A}$ (resp. $\bar{N})$ be the image of $A$ (resp. $N)$ in $\operatorname{Out}(G)$.

Theorem 1.3. Assume $G$ is irreducible. The natural map $\bar{A} \rightarrow \Gamma$ is surjective and admits a section, thus $\bar{A} \simeq \bar{N} \rtimes \Gamma$.

Theorem 1.3 can be reformulated as an equivariance property of $\chi_{V}$ : there exists an injection $\bar{\iota}_{V}: \Gamma \rightarrow \operatorname{Out}(G)$ such that $\chi_{V} \circ \bar{\iota}_{V}(\gamma)=\gamma\left(\chi_{V}\right)$.

It is actually a special case of an equivariance property that can be proved for almost any character. We call a representation rational if it admits a (matrix) model over $\mathbb{Q}$.

Theorem 1.4. Assume $G$ is irreducible. For any irreducible character $\chi$ of $G$, for all but eight exceptions, there exists an injective homomorphism: $\Gamma \stackrel{\bar{\iota}_{\chi}}{\longrightarrow} \operatorname{Out}(G)$ such that for any $\gamma \in \Gamma$, we have $\chi \circ \bar{\iota}_{\chi}(\gamma)=\gamma(\chi)$.

The exceptions are the characters of the four (rational) 5-dimensional representations of $G_{27}$, two of the 3 rational representations of dimension 6 of $G_{29}$, and the two rational representations of dimension 120 of $G_{34}$. In these cases, the complex conjugation $\mathfrak{c}$ is an element of $\Gamma$, and its image in any injective morphism $\Gamma \rightarrow \operatorname{Out}(G)$ will exchange in pairs the mentioned characters.

For an exceptional group (and a non-exceptional representation), or an imprimitive group $G(d e, e, r)$ where $\operatorname{gcd}(e, r) \leq 2$, there is a unique way to take the injection $\bar{\iota}_{\chi}$ independent of $\chi$; we will call $\bar{\iota}$ the common value. In the remaining cases, we can make choices of $\bar{\iota}_{\chi}$ which are in a single orbit for $\bar{N}$-conjugacy.

The numbers above refer to the Shephard-Todd classification of irreducible complex reflection groups.

Theorem 1.4 has the following consequence, which can be formulated without exception and without assuming $G$ is irreducible. 
Corollary 1.5. Let $G$ be any finite complex reflection group, and let $\chi$ be an irreducible character of $G$. If, for all $a \in \operatorname{Out}(G)$ we have $\chi \circ a=\chi$, then $\chi$ takes its values in $\mathbb{Q}$.

The map $\bar{\iota}$ can "almost" be lifted to $\operatorname{Aut}(G)$. More precisely, we have

Theorem 1.6. For $G$ irreducible and $\chi$ outside the exceptions of Theorem 1.4, there exists an extension $K^{\prime}$ of $K$, which is abelian over $\mathbb{Q}$ and at most quadratic over $K$, and an injective homomorphism $\tilde{\iota}_{\chi}: \operatorname{Gal}\left(K^{\prime} / \mathbb{Q}\right) \rightarrow \operatorname{Aut}(G)$ such that the composition of $\tilde{\iota}_{\chi}$ with the natural epimorphism $\operatorname{Aut}(G) \rightarrow \operatorname{Out}(G)$ factors through $\Gamma$ and induces $\bar{\iota}_{\chi}$.

One may take $K^{\prime}=K$ except when $G$ is $G_{27}$ or $G$ is a dihedral group $G(e, e, 2)$ such that $\mathbb{Q}\left(\zeta_{e}\right)$ contains no quadratic imaginary extension of $\mathbb{Q}$.

The map $\tilde{\iota}_{\chi}$ can be taken independent of $\chi$ in the same circumstances as $\bar{\iota}_{\chi}$; in such a case we will write $\tilde{\imath}$ for the common value.

Suppose we can find a model over some field $L$ of a faithful representation $\rho$ of $G$ such that $\rho(G)$ as a set is globally invariant by $\operatorname{Gal}(L / \mathbb{Q})$. Then any $\gamma \in$ $\operatorname{Gal}(L / \mathbb{Q})$ induces a permutation of $\rho(G)$ and thus a permutation on $G$, which is an automorphism. We thus get a homomorphism $\tilde{\iota}: \operatorname{Gal}(L / \mathbb{Q}) \rightarrow \operatorname{Aut}(G)$. If $L=K^{\prime}$, this $\tilde{\iota}$ is equivariant with respect to the character of $\rho$ thus is suitable for the $\tilde{\iota}$ of Theorem 1.6.

Given a homomorphism $\operatorname{Gal}(L / \mathbb{Q}) \stackrel{\tilde{\imath}}{\rightarrow} \operatorname{Aut}(G)$, and a model of an arbitrary representation $\rho$ over $L$, we say that the model is $\tilde{\imath}$-equivariant if for any $\gamma \in$ $\operatorname{Gal}(L / \mathbb{Q})$ and any $g \in G$, we have $\rho(\tilde{\iota}(\gamma)(g))=\gamma(\rho(g))$. Note that this implies the global invariance of $\rho(G)$ under $\operatorname{Gal}(L / \mathbb{Q})$. Considerations as above led us to check the following theorem:

Theorem 1.7. Let $G$ be an irreducible finite complex reflection group which is not $G_{22}$ and let $K^{\prime}$ and $\tilde{\iota}_{\chi}$ be as in Theorem 1.6. Let $\rho$ be any irreducible representation of $G$, with character $\chi$, which is not an exception in Theorem 1.4. Then there is a model of $\rho$ over $K^{\prime}$ which is $\tilde{\iota}_{\chi}$-equivariant.

In the case of $G_{22}$, we have $K=K^{\prime}=\mathbb{Q}(\mathrm{i}, \sqrt{5})$. There is no globally invariant model over $K^{\prime}$ of the reflection representation. We need to replace $K^{\prime}$ by the extension $K^{\prime \prime}=\mathbb{Q}\left(e^{\frac{2 i \pi}{20}}\right)$ to get an invariant model (then $K^{\prime \prime}$ also works for any other representation). This example illustrates the fact that asking for a $\tilde{\iota}_{\chi}$-equivariant model is stronger than merely asking as in Theorem 1.6 for a model such that $\chi \circ \tilde{\iota}_{\chi}(\gamma)=\gamma(\chi)$ where $\chi$ is the character of $\rho$.

Theorem 1.7 has the following consequence (which could have been observed from the explicit values of the invariants discovered by various authors, mostly in the nineteenth century). In this proposition we take $K^{\prime \prime}$ as above for $G_{22}$, and in the other cases we let $K^{\prime \prime}=K^{\prime}$.

Corollary 1.8. Let $G \subset \mathrm{GL}(V)$ be any irreducible complex reflection group where $V$ is a $K^{\prime \prime}$-vector space, where $K^{\prime \prime}$ is as above. There is $\mathbb{Q}$-form $V=V_{0} \otimes_{\mathbb{Q}} K^{\prime \prime}$ such that the fundamental invariants of $G$ can be taken rational, i.e., in the symmetric algebra of the dual of $V_{0}$.

Let $V^{\text {reg }}$ be the complement in $V$ of the reflecting hyperplanes for $G$. The fundamental group of the variety $V^{\mathrm{reg}} / G$ is the braid group of $G$. Since the variety $V / G$ is an affine space it is defined over $\mathbb{Q}$. A geometric reformulation of Corollary 
1.8 is that the morphism $V \rightarrow V / G$ is also defined over $\mathbb{Q}$. We deduce from Corollary 1.8 the following.

Corollary 1.9. The varieties $V^{\mathrm{reg}}, V^{\mathrm{reg}} / G$ as well as the quotient morphism $V^{\text {reg }} \rightarrow V^{\text {reg }} / G$ are defined over $\mathbb{Q}$.

The techniques we use for checking Theorem 1.7 enable us also to give a shorter proof of the Benard-Bessis theorem (see Remark 9.6).

Finally, we extend our results to a large class of non-irreducible finite complex reflection groups. We say that a group $G$ is indecomposable if it admits no nontrivial decomposition as a direct product. We show

Proposition 1.10. An irreducible complex reflection group has a decomposition $G=Z \times \hat{G}$ where $Z \subseteq Z G$, and where $\hat{G}$ is indecomposable non-abelian or trivial. In this decomposition $Z$ is unique.

We call $\hat{G}$ the non-abelian factor of $G$, denoted $\operatorname{Nab}(G)$; it is unique up to isomorphism, since $Z$ is unique. We then show:

Theorem 1.11. Let $G=G_{1} \times \ldots \times G_{n}$ be the decomposition in irreducible factors of a complex reflection group $G \subset \mathrm{GL}(V)$. Then the following are equivalent:

- Any automorphism of $G$ is the product of a central automorphism and of an automorphism which preserves the reflections.

- No $G_{i}$ is isomorphic to $\mathfrak{S}_{6}$, and the decomposition has the property that for any $i, j$, we have $\operatorname{Nab}\left(G_{i}\right) \simeq \operatorname{Nab}\left(G_{j}\right) \Rightarrow G_{i} \simeq G_{j}$.

We give the list of central and non-abelian factors of irreducible groups in Table3.

\section{BACKGROUND ON THE INFINITE SERIES}

We will use the following notation throughout this paper. $V$ is a vector space over the subfield $K$ of $\mathbb{C}$. $G$ is a finite subgroup of $\mathrm{GL}(V)$ generated by (pseudo)reflections. We assume that $K$ is the field of definition of $G$, that is, it is the subfield of $\mathbb{C}$ generated by the traces of the elements of $G$. The group $G$ is irreducible if the representation $V$ of $G$ is irreducible.

We denote by $g \mapsto$ Ad $g$ the map which maps $g \in G$ to the inner automorphism $x \mapsto{ }^{g} x=g x g^{-1}$, and we denote $x^{g}$ for $g^{-1} x g$. For two linear representations $\rho_{1}, \rho_{2}$ : $G \rightarrow \mathrm{GL}\left(\mathbb{C}^{n}\right)$ we note $\rho_{1}=\rho_{2}$ when they represent the same homomorphism, and $\rho_{1} \simeq \rho_{2}$ when they are isomorphic, that is, their characters are the same.

The Shephard-Todd classification of irreducible finite reflection groups (see e.g. Cohen) shows that there is one infinite series, identified by three positive integer parameters $d, e$ and $r$, and denoted by $G(d e, e, r)$. In addition, there are 34 exceptional ones, denoted $G_{4}$ to $G_{37}$.

For the convenience of the reader, and for future reference, we review the definition and some properties of the groups $G(d e, e, r)$,

2.1. The groups $G(d e, e, r)$. We will denote by $\mu_{n}$ the subgroup of $n$-th roots of unity in $\mathbb{C}^{\times}$, and by $\zeta_{n}$ the primitive root of unity $e^{2 i \pi / n}$.

The group $G(d e, e, r)$ is defined as the subgroup of $\mathrm{GL}_{r}(\mathbb{C})$ consisting of $r$ by $r$ monomial matrices with entries in $\mu_{d e}$, and such that the product of non-zero entries is in $\mu_{d}$; this defines an irreducible reflection group except for the case of $\mathfrak{S}_{r}=G(1,1, r)$, where one has to quotient by the one-dimensional fixed points, and for $G(2,2,2) \simeq \mathfrak{S}_{2}^{2}$. 
The field of definition $K$ of $G(d e, e, r)$ is the field generated by the traces of elements of $G(d e, e, r)$. We get $K=\mathbb{Q}\left(\zeta_{d e}\right)$, except when $d=1$ and $r=2$. In this last case, $G(e, e, 2)$ is the dihedral group of order $2 e$, whose field of definition is $K=\mathbb{Q}\left(\cos \left(\frac{2 \pi}{e}\right)\right)=\mathbb{Q}\left(\zeta_{e}+\zeta_{e}^{-1}\right)$.

The group $\mathfrak{S}_{r}=G(1,1, r)$ is a subgroup of $G(d e, e, r)$; if we denote by $D(d e, e, r)$ the subgroup of diagonal matrices in $G(d e, e, r)$, we have a semi-direct product decomposition $G(d e, e, r)=D(d e, e, r) \rtimes \mathfrak{S}_{r}$.

The group $G(d, 1, r)$ is generated by the set of reflections $\left\{t, s_{1}, \ldots, s_{r-1}\right\}$ where $t$ is the matrix $\operatorname{Diag}\left(\zeta_{d}, 1, \ldots, 1\right) \in D(d, 1, r)$ and $s_{k} \in \mathfrak{S}_{r}$ is the permutation matrix corresponding to the transposition $(k, k+1)$.

The group $G(d e, e, r)$ is a normal subgroup of index $e$ in $G(d e, 1, r)$; it is generated by the set of reflections $\left\{t^{\prime}, s_{1}^{\prime}, s_{1}, \ldots, s_{r-1}\right\}$ where $t^{\prime}=t^{e}$ and $s_{1}^{\prime}=s_{1}^{t}$; here $t$ refers to the generator $t=\operatorname{Diag}\left(\zeta_{d e}, 1, \ldots, 1\right)$ of $G(d e, 1, r)$. The generator $t^{\prime}$ should be omitted (being trivial) if $d=1$ and $s_{1}^{\prime}$ should be omitted (being unnecessary) if $e=1$. The quotient $G(d e, 1, r) / G(d e, e, r)$ is cyclic, generated by the image of $t$.

2.2. Presentations. The following diagram gives a presentation of $G(d, 1, r)$ :

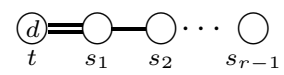

which means that $t, s_{1}, \ldots, s_{r-1}$ satisfy the same braid relations as the Coxeter group $W\left(B_{r}\right)$ (actually the braid group of $G(d, 1, r)$ is isomorphic to that of $W\left(B_{r}\right)$, see BMR $)$, and that one gets a presentation of $G(d, 1, r)$ by adding the order relations $t^{d}=1$ and $s_{i}^{2}=1$.

For $G(d e, e, r)$, to get a presentation of the braid group one needs to replace the relation $t s_{1} t s_{1}=s_{1} t s_{1} t$ implied by the above diagram by the following braid relations involving $t^{\prime}$ and $s_{1}^{\prime}$ :

$$
\begin{aligned}
& t^{\prime} s_{1}^{\prime} s_{1}=s_{1}^{\prime} s_{1} t^{\prime}, \\
& s_{1}^{\prime} s_{1} s_{2} s_{1}^{\prime} s_{1} s_{2}=s_{2} s_{1}^{\prime} s_{1} s_{2} s_{1}^{\prime} s_{1}, \\
& \underbrace{s_{1} t^{\prime} s_{1}^{\prime} s_{1} s_{1}^{\prime} s_{1} \ldots}_{e+1}=\underbrace{t^{\prime} s_{1}^{\prime} s_{1} s_{1}^{\prime} s_{1} \ldots}_{e+1}
\end{aligned}
$$

and one gets a presentation of $G(d e, e, r)$ by adding the order relations $t^{\prime d}=1$ and $s_{1}^{\prime 2}=s_{i}^{2}=1$.

2.3. The representations of $G(d, 1, r)$. We recall from ArKo explicit models over $K=\mathbb{Q}\left(\zeta_{d}\right)$ for the irreducible representations of $G(d, 1, r)$. Let $\mathcal{L}$ be the set of $d$-tuples $\boldsymbol{\lambda}=\left(\lambda_{0}, \ldots, \lambda_{d-1}\right)$ of partitions with total size $r$. We denote by $\mathcal{T}(\boldsymbol{\lambda})$ the set of standard tableaux of shape $\boldsymbol{\lambda}$, that is, the set of tuples $\mathbf{T}=\left(T_{0}, \ldots, T_{d-1}\right)$ such that $T_{i}$ is a filling of the Young diagram of $\lambda_{i}$ with numbers in $[1, r]$, with the conditions that each of these numbers appears (exactly once) in one of the filled diagrams, and that they are increasing across the rows and columns of each $T_{i}$. For $m \in[1, r]$ we let $\mathbf{T}(m)=i$ if $m$ is placed in $T_{i}$.

To $\boldsymbol{\lambda} \in \mathcal{L}$ we associate the $\mathbb{Q}$-vector space $V^{0}(\boldsymbol{\lambda})$ of basis $\mathcal{T}(\boldsymbol{\lambda})$, and define $V(\boldsymbol{\lambda})=V^{0}(\boldsymbol{\lambda}) \otimes \mathbb{Q}\left(\zeta_{d}\right)$. Explicit formulas in ArKo describe a representation $\rho_{\boldsymbol{\lambda}}$ of $G(d, 1, r)$ over $V(\boldsymbol{\lambda})$, and the $\rho_{\boldsymbol{\lambda}}$ provide a complete set of representatives for the irreducible representations of $G(d, 1, r)$ (see ArKo corollary 3.14).

- The matrix $\rho_{\boldsymbol{\lambda}}(t)$ is given by $\rho_{\boldsymbol{\lambda}}(t) \mathbf{T}=\zeta_{d}^{\mathbf{T}(1)} \mathbf{T}$. 
- The matrices $\rho_{\boldsymbol{\lambda}}\left(s_{i}\right)$ for $1 \leq i \leq n-1$ are rational in the basis $\mathcal{T}(\boldsymbol{\lambda})$, thus belong to $\operatorname{GL}\left(V^{0}(\boldsymbol{\lambda})\right)$. To describe them we introduce some notation: given a tuple of tableaux $\mathbf{T}$, let $\mathbf{T}_{i \leftrightarrow i+1}$ be the tuple obtained by exchanging the numbers $i$ and $i+1$ in $\mathbf{T}$ when this is still a tuple of standard tableaux, and 0 otherwise (this last case will only occur when the numbers $i$ and $i+1$ occur in the same tableau and on either the same line or the same column). Then, if $i$ and $i+1$ occur in the same tableau, define their axial distance $a(i, i+1)$ to be the distance between the diagonals where they occur (more precisely, if $i$ occurs at coordinates $i_{0}, i_{1}$ and $i+1$ at coordinates $j_{0}, j_{1}$ we set $\left.a(i, i+1)=\left(i_{0}-i_{1}\right)-\left(j_{0}-j_{1}\right)\right)$. It is clear that for a standard tableau we always have $a(i, i+1) \neq 0$. Finally, take the convention that when $i$ and $i+1$ do not occur in the same tableau, then $a(i, i+1)=\infty$ so that $1 / a(i, i+1)=0$. Then the formula is:

$$
\rho_{\boldsymbol{\lambda}}\left(s_{i}\right) \mathbf{T}=\frac{1}{a(i, i+1)} \mathbf{T}+\left(1+\frac{1}{a(i, i+1)}\right) \mathbf{T}_{i \leftrightarrow i+1} .
$$

2.4. The representations of $G(d e, e, r)$. As in $\mathrm{Ar}$, we will get the irreducible representations of $G(d e, e, r)$ by Clifford theory, as components of the restriction of $\rho_{\boldsymbol{\lambda}} \in \operatorname{Irr}(G(d e, 1, r))$ to $G(d e, e, r)$.

We recall that $\left\{t^{e}, s_{1}^{t}, s_{1}, \ldots, s_{r-1}\right\}$ are the generating reflections for $G(d e, e, r)$, and that the quotient $G(d e, 1, r) / G(d e, e, r)$ is cyclic, generated by $t$. Let $\chi$ be the generator of the group of linear characters of the quotient which maps $t$ to $\zeta_{e}$. From the formulae for the action of $t$ and $s_{i}$, we see that there is a permutation $\sigma(\boldsymbol{\lambda})$ of the tuple $\boldsymbol{\lambda}$ such that $\chi \otimes \rho_{\boldsymbol{\lambda}} \simeq \rho_{\sigma(\boldsymbol{\lambda})}$; it is given by $\sigma(\boldsymbol{\lambda})=\left(\lambda_{d}, \lambda_{d+1}, \ldots, \lambda_{d+d e-1}\right)$ (where the indices are taken $(\bmod d e))$. Let $\langle\sigma\rangle$ be the cyclic group generated by $\sigma$, and let $C_{\boldsymbol{\lambda}}=\left\langle\sigma^{b}\right\rangle$ be the subgroup which stabilizes $\boldsymbol{\lambda}$. By Clifford theory, the representation $\rho_{\boldsymbol{\lambda}}$ restricts to $G(d e, e, r)$ as the sum of $\# C_{\boldsymbol{\lambda}}=e / b$ distinct irreducible representations.

We can actually define an operator $S$ on $V(\boldsymbol{\lambda})$ associated to $\sigma^{b}$ whose eigenspaces will be the irreducible constituents. For $\mathbf{T}=\left(T_{0}, \ldots, T_{d e-1}\right)$ we set $S(\mathbf{T})=$ $\left(T_{b d}, T_{b d+1}, \ldots, T_{b d+d e-1}\right)$. Since $\sigma^{b}(\boldsymbol{\lambda})=\boldsymbol{\lambda}$, this is another tuple of tableaux of shape $\boldsymbol{\lambda}$. It is easy to check that $S$ commutes with $\rho_{\boldsymbol{\lambda}}\left(s_{i}\right)$ and with $\rho_{\boldsymbol{\lambda}}\left(s_{1}^{t}\right)$, and that $S \circ \rho_{\boldsymbol{\lambda}}(t)=\zeta_{e}^{b} \rho_{\boldsymbol{\lambda}}(t) \circ S$. It follows that $S$ commutes with the action of $G(d e, e, r)$ and its eigenspaces $V(\boldsymbol{\lambda}, \omega)=\operatorname{Ker}(S-\omega)$ for $\omega \in \mu_{\# C_{\boldsymbol{\lambda}}}$ afford irreducible representations of $G(d e, e, r)$. We denote by $\rho_{\boldsymbol{\lambda}, \omega}$ the representation afforded by $V(\boldsymbol{\lambda}, \omega)$; it is clear that

$$
p_{\omega}:=\frac{1}{\# C_{\boldsymbol{\lambda}}} \sum_{i=0}^{\# C_{\boldsymbol{\lambda}}-1} \omega^{-i} S^{i}
$$

is the $G(d e, e, r)$-invariant projector on $V(\boldsymbol{\lambda}, \omega)$.

From this we get, if we denote $\chi_{\boldsymbol{\lambda}, \omega}$ the character of $\rho_{\boldsymbol{\lambda}, \omega}$ :

$$
\chi_{\boldsymbol{\lambda}, \omega}(g)=\frac{1}{\# C_{\boldsymbol{\lambda}}} \sum_{i=0}^{\# C_{\boldsymbol{\lambda}}-1} \omega^{-i} \operatorname{Trace}\left(\rho_{\boldsymbol{\lambda}}(g) S^{i}\right) .
$$

We will now want to describe a model of $\rho_{\boldsymbol{\lambda}, \omega}$. We first recall Ariki's model (see [Ar, section 2]), and then introduce another one, simpler for our purpose. Ariki chooses a basis of $V(\boldsymbol{\lambda}, \omega)$ given by the $p_{\omega}(\mathbf{T})$, where he chooses for $\mathbf{T}$ representatives of the $S$-orbits on $\mathcal{T}$ given by the subset $\mathcal{T}_{0}$ of tuples of tableaux which satisfy $\mathbf{T}(1)<b d$. Setting $\mathbf{T}^{(\omega)}=p_{\omega}(\mathbf{T})$, we then get formulas for the action of the generators of $G(d e, e, r)$ on the basis $\left\{\mathbf{T}^{(\omega)}\right\}_{\mathbf{T} \in \mathcal{T}_{0}}$. We will write $\theta$ for $\zeta_{e}^{b}$ to 
simplify notation. Using that $\rho_{\boldsymbol{\lambda}}(t) p_{\omega}=p_{\omega \theta} \rho_{\boldsymbol{\lambda}}(t)$, we get $\rho_{\boldsymbol{\lambda}}(t) \mathbf{T}^{(\omega)}=\zeta_{d e}^{\mathbf{T}(1)} \mathbf{T}^{(\omega \theta)}$ from which we get

$$
\rho_{\boldsymbol{\lambda}, \omega}\left(t^{e}\right) \mathbf{T}^{(\omega)}=\zeta_{d}^{\mathbf{T}(1)} \mathbf{T}^{(\omega)} .
$$

To write the formula for $s_{i}$, first note that if $\mathbf{T} \in \mathcal{T}_{0}$, then $\mathbf{T}_{i \leftrightarrow i+1}$ is not in general in $\mathcal{T}_{0}$ when $i=1$. We have to take $\mathbf{T}_{1 \leftrightarrow 2}^{\prime}=S^{\left\lfloor\frac{\mathbf{T}^{(2)}}{b d}\right\rfloor} \mathbf{T}_{1 \leftrightarrow 2}$ to get an element of $\mathcal{T}_{0}$. Using that $p_{\omega}\left(\mathbf{T}_{1 \leftrightarrow 2}^{\prime}\right)=\omega^{\left\lfloor\frac{\mathbf{T}(2)}{b d}\right\rfloor} p_{\omega}\left(\mathbf{T}_{1 \leftrightarrow 2}\right)$, we get for $i>1$,

$$
\rho_{\boldsymbol{\lambda}, \omega}\left(s_{i}\right) \mathbf{T}^{(\omega)}=\frac{1}{a(i, i+1)} \mathbf{T}^{(\omega)}+\left(1+\frac{1}{a(i, i+1)}\right) \mathbf{T}_{i \leftrightarrow i+1}^{(\omega)}
$$

and

$$
\rho_{\boldsymbol{\lambda}, \omega}\left(s_{1}\right) \mathbf{T}^{(\omega)}=\frac{1}{a(1,2)} \mathbf{T}^{(\omega)}+\left(1+\frac{1}{a(1,2)}\right) \omega^{-\left\lfloor\frac{\mathbf{T}(2)}{b d}\right\rfloor} \mathbf{T}_{1 \leftrightarrow 2}^{\prime(\omega)} .
$$

Finally, using the above formulas we get

$$
\rho_{\boldsymbol{\lambda}, \omega}\left(s_{1}^{t}\right) \mathbf{T}^{(\omega)}=\zeta_{d e}^{\mathbf{T}(1)-\mathbf{T}(2)} \rho_{\boldsymbol{\lambda}, \omega}\left(s_{1}\right) \mathbf{T}^{(\omega)} .
$$

We now introduce a model which does not depend on a choice of representatives $\mathcal{T}_{0}$, using the equality $p_{\omega \theta^{-1}} \circ \rho_{\boldsymbol{\lambda}}(g)=p_{\omega} \rho_{\boldsymbol{\lambda}}(\operatorname{Ad} t(g))$ which implies $\chi_{\lambda, \theta^{i}}(g)=$ $\chi_{\lambda, 1}\left(\operatorname{Ad}\left(t^{-i}\right) g\right)$. We choose the same model as Ariki of $\rho_{\lambda, 1}$, except that, as the image by $p_{1}$ of a tuple $\mathbf{T}$ is the same as that of all elements of the same $S$-orbit, we take as basis elements the averages of $S$-orbits. We denote by $(\mathbf{T})$ the average of the $S$-orbit of $\mathbf{T}$. We thus get a basis $\{(\mathbf{T})\}_{(\mathbf{T}) \in \mathcal{T}(\boldsymbol{\lambda}) / S}$ of $V(\boldsymbol{\lambda}, 1)$. We take the same basis for $V\left(\boldsymbol{\lambda}, \theta^{i}\right)$ and define $\rho_{\boldsymbol{\lambda}, \theta^{i}}(g):=\rho_{\boldsymbol{\lambda}, 1}\left(\operatorname{Ad}(t)^{-i} g\right)$. We now have the following formulas for $\rho_{\boldsymbol{\lambda}, 1}$ :

$$
\begin{aligned}
\rho_{\boldsymbol{\lambda}, 1}\left(t^{e}\right)(\mathbf{T}) & =\zeta_{d}^{\mathbf{T}(1)}(\mathbf{T}), \\
\rho_{\boldsymbol{\lambda}, 1}\left(s_{i}\right)(\mathbf{T}) & =\frac{1}{a(i, i+1)}(\mathbf{T})+\left(1+\frac{1}{a(i, i+1)}\right)\left(\mathbf{T}_{i \leftrightarrow i+1}\right) \text { for any } i, \\
\rho_{\boldsymbol{\lambda}, 1}\left(s_{1}^{t^{k}}\right)(\mathbf{T}) & =\zeta_{d e}^{k(\mathbf{T}(1)-\mathbf{T}(2))} \rho_{\boldsymbol{\lambda}, 1}\left(s_{1}\right)(\mathbf{T})
\end{aligned}
$$

(note that the above formulas make sense since both $\zeta_{d}^{\mathbf{T}(1)}$ and $\zeta_{d e}^{\mathbf{T}(1)-\mathbf{T}(2)}$ are constant on a given $S$-orbit).

\section{The GROUP $\bar{N}$}

For $G$ irreducible, the subgroup $\bar{N}$ of $\operatorname{Out}(G)$ induced by $N_{\mathrm{GL}(V)}(G)$ has been determined in BMM, 3.13]. For the convenience of the reader we recall here the result. When $K$ is a real field, $G$ is a finite Coxeter group and elements of $\bar{N}$ correspond to automorphisms of the Coxeter diagram. Such automorphisms are traditionally denoted by an exponent, for example, ${ }^{3} D_{4}$ denotes an automorphism of order 3 of the group $W\left(D_{4}\right)=G(2,2,4)$. We will use a similar notation for the other cases. With one exception ${ }^{2} F_{4}$, all such automorphisms result from a normal embedding of $G$ into another reflection group of the same rank (the case of ${ }^{2} F_{4}$ results from the embedding $G_{28}=W\left(F_{4}\right) \subset G_{31}$, which is not normal). We 
indicate at the same time the relevant embedding.

\begin{tabular}{|l|c|}
\hline Automorphism & Embedding \\
\hline${ }^{i} G(d e, e, r)$ where $i>1, i \mid d e$ & $G(d e, e, r) \triangleleft G(d e, 1, r)$ \\
${ }^{3} G(4,2,2)$ & $G(4,2,2) \triangleleft G_{6}$ \\
${ }^{4} G(3,3,3)$ & $G(3,3,3) \triangleleft G_{26}$ \\
${ }^{3} D_{4}$ & $G(2,2,4)=W\left(D_{4}\right) \triangleleft G_{28}=W\left(F_{4}\right)$ \\
${ }^{2} G_{5}$ & $G_{5} \triangleleft G_{14}$ \\
${ }^{2} G_{7}$ & $G_{7} \triangleleft G_{15}$ \\
${ }^{2} F_{4}$ & \\
\hline
\end{tabular}

This table needs a few comments on how it describes the group $\bar{N}$.

The automorphisms of $G(d e, e, r)$ induced by $G(d e, 1, r)$ are induced by $\operatorname{Ad} t$, where $t=\operatorname{Diag}\left(\zeta_{d e}, 1, \ldots, 1\right)$. Let us determine when the endomorphism Ad $t^{i}$ of $G=G(d e, e, r)$ is inner. This happens if and only if there exists $g \in G$ such that $t^{-i} g$ is scalar. Since $t^{i}=\operatorname{Diag}\left(\zeta_{d e}^{i}, 1, \ldots, 1\right)$, the element $g$ must be of the form $\operatorname{Diag}\left(\zeta_{d e}^{i} a, a, \ldots, a\right)$ for some $a \in \mu_{d e}$ and the condition that $g \in G$ is $a^{r} \zeta_{d e}^{i} \in \mu_{d}$, i.e., $\zeta_{d e}^{i} \in \mu_{d} \cdot \mu_{d e}^{r}=\mu_{d e}^{e} \cdot \mu_{d e}^{r}=\mu_{d e}^{\operatorname{gcd}(e, r)}$, i.e., we get that $\operatorname{Ad} t^{i}$ is inner if and only if $i$ is multiple of $\operatorname{gcd}(e, r)$. Thus the image of $\operatorname{Ad} t$ in $\operatorname{Out}(G)$ is of order $\operatorname{gcd}(e, r)$.

For $G=G(2,2,4)=D_{4}$ the group $\bar{N}$ is generated by ${ }^{3} D_{4}$ and ${ }^{2} G(2,2,4)$ and is isomorphic to $\mathfrak{S}_{3}$.

For $G=G(4,2,2)$ the group $\bar{N}$ is generated by ${ }^{3} G(4,2,2)$ and ${ }^{2} G(4,2,2)$ and is isomorphic to $\mathfrak{S}_{3}$.

For $G=G(3,3,3)$ the group $\bar{N}$ is generated by ${ }^{3} G(3,3,3)$ and the image in Out $(G)$ of ${ }^{4} G(3,3,3)$, which is of order 2 , and is isomorphic to the alternating group $\mathfrak{A}_{4}$.

The determination of $\bar{N}$ has the following consequence.

Proposition 3.1. The natural action of $\operatorname{Out}(G)$ on $\operatorname{Irr}(G)$ is faithful.

Proof. We note that an element of $\operatorname{Out}(G)$ which acts trivially on $\operatorname{Irr}(G)$ belongs to $\bar{N}$, as it fixes $\chi_{V}$. Assume that $G=G(d e, e, r)$, let $v=\operatorname{gcd}(e, r)$ and consider the de-tuple of partitions $\boldsymbol{\lambda}=\left(\lambda_{0}, \ldots, \lambda_{d e}\right)$ where $\lambda_{i}=\{r / v\}$ if $i$ is a multiple of $d e / v$ and $\lambda_{i}=\emptyset$ otherwise. With the notations of subsection 2.4 the tuple $\boldsymbol{\lambda}$ is stabilized by $\sigma^{e / v}$ which corresponds to $(\operatorname{Ad} t)^{e / v}$, thus the restriction to $G$ of $\rho_{\boldsymbol{\lambda}} \in \operatorname{Irr} G(d e, 1, r)$ has an inertia group of cardinality $v$, hence the subgroup of $\bar{N}$ induced by $\operatorname{Ad} t$ acts faithfully on its set of irreducible components. It thus remains to consider the special cases mentioned above. Inspection of the character tables, verifying that they are not left invariant by the automorphisms in these special cases, concludes the proof.

\section{The structure of $\operatorname{Aut}(G)$}

In this section we show that for an irreducible $G$, every automorphism is the product of a central automorphism and an automorphism which preserves the reflections (except for $G=G(1,1,6)=\mathfrak{S}_{6}$ ). Since, for $G$ of rank at least 3, a non-trivial central automorphism does not preserve the reflections, this will prove Theorem 1.1.

If $G \subset \mathrm{GL}(V)$ is an irreducible complex reflection group, we may identify $Z G$ to a subgroup of $\mathbb{C}^{\times}$, thus $C$ is formed of the automorphisms $\alpha_{\chi}$ of the form $\alpha_{\chi}(g)=g \chi(g)^{-1}$ where $\chi \in \operatorname{Hom}\left(G, \mathbb{C}^{\times}\right)$is a linear character. This defines in 
general an endomorphism of $G$ if the values of $\chi$ lie in $Z G$. It is an automorphism (thus a central automorphism) if and only if $z \neq \chi(z)$ for all $z \in Z G-\{1\}$.

\subsection{The infinite series.}

Proposition 4.1. Any automorphism of a group $G(d e, e, r)$ is composed of an automorphism which preserves the reflections with a central automorphism, except for the case of $G(1,1,6)=\mathfrak{S}_{6}$ and for the case of the non-irreducible group $G(2,2,2) \simeq \mathfrak{S}_{2} \times \mathfrak{S}_{2}$.

We first need a lemma.

Lemma 4.2. The group $D=D(d e, e, r)$ of diagonal matrices in $G=G(d e, e, r)$ is a characteristic subgroup of $G$ if and only if $G$ is not one of the groups $G(2,2,2)$, $G(2,1,2), G(4,2,2), G(3,3,3), G(2,2,4)$.

Proof. We show first that for $r \geq 5$ the group $D$ is the unique maximal normal abelian subgroup of $G$. Let $E$ be another abelian normal subgroup. Using the semi-direct product decomposition $G=D \rtimes \mathfrak{S}_{r}$, the image $E /(E \cap D)$ of $E$ in $\mathfrak{S}_{r}$ is an abelian normal subgroup of $\mathfrak{S}_{r}$. Since the only such subgroup of $\mathfrak{S}_{r}$ is trivial if $r \geq 5$, we get that $E /(E \cap D)=1$ meaning $E \subseteq D$.

We now assume $r \leq 4$. If $d=e=1$, then $G=\mathfrak{S}_{r}$ and $D=1$ is characteristic. We thus assume now de $>1$. We will show that $D$ is the unique normal abelian subgroup of maximal order, except for a few special cases that we handle separately. As before we exclude the non-irreducible and abelian case $G=G(2,2,2)$, for which $\operatorname{Out}(G)=\mathrm{GL}_{2}\left(\mathbb{F}_{2}\right)$ does not preserve $D \simeq \mathbb{Z} / 2$. We note for future use that the order of the center $Z G$ is the $\operatorname{gcd}$ of the reflection degrees of $G$, equal to $d \cdot \operatorname{gcd}(e, r)$, and that $D$ is of order $d^{r} e^{r-1}$ (one chooses arbitrarily $r-1$ eigenvalues in $\mu_{d e}$, and then there are $d$ possibilities for the last one such that the product is in $\mu_{d}$ ).

For $r=1$ we have $G=D$ thus there is nothing to prove. Let $E$ be an abelian normal subgroup of $G$ whose image in $\mathfrak{S}_{r}$ is non-trivial. For $1<r \leq 4$, the only non-trivial abelian normal subgroup $K$ of $\mathfrak{S}_{r}$ is transitive and has order $r$. It follows that the image of $E$ in $\mathfrak{S}_{r}$ is $K$ and is therefore transitive. Let $x \in E \cap D$ be a diagonal matrix in $E$, with diagonal entries $\alpha_{1}, \ldots, \alpha_{r}$. For $g \in E$ with image $w \in K \subset \mathfrak{S}_{r}, x=g x g^{-1}$ is a diagonal matrix with entries $\alpha_{w(1)}, \ldots, \alpha_{w(r)}$. Since $K$ is transitive we get $\alpha_{1}=\cdots=\alpha_{r}$ hence $x \in Z G$ and $E \cap D \subset Z G$. It follows that $E$ is an extension of $K$ by a subgroup of $Z G$. In particular, \#E $\leq$ $(\# Z G)(\# K)=r d \operatorname{gcd}(e, r)$. Since $\# D=d^{r} e^{r-1}$, it is then straightforward to check that \#E<\#D, except for $G$ one of $G(4,4,2), G(2,1,2), G(4,2,2), G(3,3,3)$, $G(2,2,4)$; hence apart from these exceptions $D$ is preserved by any automorphism of $G$. A check shows that the outer automorphism of $G(2,1,2)$ does not preserve the diagonal matrices, but that it does preserve them in the incarnation $G(4,4,2)$; and that $G(4,2,2), G(3,3,3), G(2,2,4)$ are genuine exceptions.

We are now in position to prove Proposition 4.1, except for the groups $G(2,1,2)$, $G(4,2,2), G(3,3,3), G(2,2,4)$, which will be dealt with in the next section. We thus assume that $G$ is not one of these exceptions, and in addition we first assume that $r \neq 6$.

Let $t$ and $s_{1}, \ldots, s_{r-1}$ be reflections as in section 2.1 such that $G$ is generated by $t^{e}, s_{1}^{t}, s_{1}, \ldots, s_{r-1}$ (if $d=1$ we drop $t^{e}$ and if $e=1$ we drop $s_{1}^{t}$ ). Let $\phi$ be an automorphism of $G$. Since we are not in one of the exceptions of the lemma, $\phi$ preserves $D$ thus induces an automorphism $\bar{\phi}$ of $\mathfrak{S}_{r}$. Since we assumed $r \neq 6$, 
the automorphism $\bar{\phi}$ is inner, of the form $\operatorname{Ad} \sigma$ for some $\sigma \in \mathfrak{S}_{r}$, and lifting $\sigma$ to a permutation matrix in $G$, we see that up to an inner automorphism we may assume that $\phi$ induces the identity on $\mathfrak{S}_{r}$, that is, preserves the shape of a monomial matrix in $G$.

We will denote by $M_{\sigma}$ the permutation matrix corresponding to $\sigma \in \mathfrak{S}_{r}$. Thus $\phi\left(s_{1}\right)$ is of the form $\operatorname{Diag}\left(x_{1}, \ldots, x_{r}\right) M_{(1,2)}$; the fact that $\phi\left(s_{1}\right)$ is an involution implies that $x_{1}=x_{2}^{-1}$ and that $x_{3}, \cdots, x_{n}$ are signs. The fact that $\phi\left(s_{1}\right)$ commutes with $\phi\left(s_{3}\right), \ldots, \phi\left(s_{r-1}\right)$ implies that $x_{3}=x_{4}=\cdots=x_{r}$. Thus $\phi\left(s_{1}\right)$ is of the form $\operatorname{Diag}\left(\alpha_{1}^{-1}, \alpha_{1}, \epsilon, \ldots, \epsilon\right) M_{(1,2)}$ for some sign $\epsilon$ and some $\alpha_{1} \in \mu_{d e}$.

Similarly, $\phi\left(s_{i}\right)$ is of the form $\operatorname{Diag}\left(\epsilon, \ldots, \alpha_{i}^{-1}, \alpha_{i}, \ldots, \epsilon\right) M_{(i, i+1)}$ where $\alpha_{i}$ is in $i$ th position (the sign $\epsilon$ is the same since $s_{1}$ and $s_{i}$ are conjugate), and $\phi\left(s_{1}^{t}\right)$ is of the form $\operatorname{Diag}\left(\alpha^{\prime-1}, \alpha^{\prime}, \epsilon^{\prime}, \ldots, \epsilon^{\prime}\right) M_{(1,2)}$. We have actually $\epsilon=\epsilon^{\prime}$ since they occur only when $r>2$ in which case $s_{1}^{t}=\left(s_{1}\right)^{s_{2} s_{1} s_{1}^{t} s_{2}}$ is conjugate to $s_{1}$.

Let $s=\operatorname{Diag}\left(1, \epsilon \alpha_{1}^{-1}, \epsilon^{2}\left(\alpha_{1} \alpha_{2}\right)^{-1}, \ldots, \epsilon^{r}\left(\alpha_{1} \ldots \alpha_{r}\right)^{-1}\right) \in G(d e, 1, r)$. Then Ad $s$ induces an automorphism of $G$ which preserves the reflections, and the composed automorphism $\phi^{\prime}=\operatorname{Ad} s \circ \phi$ satisfies $\phi^{\prime}\left(s_{1}\right)=\epsilon s_{1}, \ldots, \phi^{\prime}\left(s_{r}\right)=\epsilon s_{r}$. The element $\phi^{\prime}\left(s_{1}^{t}\right)$ is of the form $\operatorname{Diag}\left(\alpha^{\prime \prime-1}, \alpha^{\prime \prime}, \epsilon, \ldots, \epsilon\right) M_{(1,2)}$ for some $\alpha^{\prime \prime} \in \mu_{d e}$.

The fact that $s_{1} s_{1}^{t}$ is of order de implies that $\epsilon \alpha^{\prime \prime}$ is a primitive $d e$-th root of unity. Thus there exists an element $\gamma \in \operatorname{Gal}\left(\mathbb{Q}\left(\zeta_{d e}\right) / \mathbb{Q}\right)$ such that $\gamma\left(\epsilon \alpha^{\prime \prime}\right)=\zeta_{d e}$. Applying $\gamma$ to the matrices of $G$ gives an automorphism which preserves the reflections, and composing $\phi^{\prime}$ with that automorphism, we get an automorphism $\phi^{\prime \prime}$ such that $\phi^{\prime \prime}\left(s_{i}\right)=\epsilon s_{i}$ and $\phi^{\prime \prime}\left(s_{1}^{t}\right)=\epsilon s_{1}^{t}$.

If $d=1$ we are finished: $\phi^{\prime \prime}$ is the central automorphism given by $s \mapsto \epsilon s$ for any reflection.

Assume now that $d>1$. Then $\phi^{\prime \prime}\left(t^{e}\right)$ is a diagonal matrix which commutes with $s_{2}, \ldots, s_{r-1}$ thus is of the form $z \operatorname{Diag}(\zeta, 1, \ldots, 1)$ for some $z \in Z G$ and some $\zeta \in \mu_{d e}$. Since it is of order $d, z$ must be of order $d$ and $\zeta$ must be a primitive $d$-th root of unity.

Assume first that $e=1$. Then we do not need $s_{1}^{t}$ among the generators, and composing $\phi^{\prime \prime}$ with the automorphism induced by some appropriate element of $\operatorname{Gal}\left(\mathbb{Q}\left(\zeta_{d e}\right) / \mathbb{Q}\right)$ we may get a $\phi^{\prime \prime \prime}$ such that $\phi^{\prime \prime \prime}\left(s_{i}\right)=\epsilon s_{i}$ and $\phi^{\prime \prime \prime}(t)=z^{\prime} t$ for some $z^{\prime} \in Z G$, thus $\phi^{\prime \prime \prime}$ is a central automorphism.

Finally, assume that $e$ and $d$ are not 1 . Then we use the third relation

$$
\underbrace{s_{1} t^{e} s_{1}^{t} s_{1} s_{1}^{t} s_{1} \ldots}_{e+1}=\underbrace{t^{e} s_{1}^{t} s_{1} s_{1}^{t} s_{1} \ldots}_{e+1}
$$

in 2.1 which implies that $\zeta=\zeta_{d}$, thus $\phi$ is the central automorphism $s_{i} \mapsto \epsilon s_{i}$ and $t^{e} \mapsto z t^{e}$.

The same method will apply when $r=6$ and $d e>1$ if we can exclude the possibility for $\bar{\phi}$ to be a non-inner automorphism of $\mathfrak{S}_{6}$. Up to conjugation by a permutation matrix, we may assume that $\bar{\phi}$ sends $(1,2)$ to $(1,2)(3,4)(5,6)$. Let $p=M_{(1,2)(3,4)(5,6)}$. We have $\phi\left(C_{G}\left(s_{1}\right) \cap D\right)=C_{G}\left(\phi\left(s_{1}\right)\right) \cap D=C_{G}(p) \cap D$, the first equality since $D$ is a characteristic subgroup. and the second since $G=$ $D \rtimes \mathfrak{S}_{6}$ and $D$ is abelian. It follows that $C_{G}\left(s_{1}\right) \cap D$ and $C_{G}(p) \cap D$ have the same cardinality. One easily gets $\#\left(C_{G}\left(s_{1}\right) \cap D\right)=(d e)^{4} d$. On the other hand, a matrix in $C_{G}(p) \cap D$ is uniquely determined by (diagonal) coefficients $(a, b, c) \in \mu_{d e}^{3}$ such that $c^{2} \in a^{-2} b^{-2} \mu_{d}$. Since an element in $\mu_{d e}$ has at most two square roots, 
it follows that $\#\left(C_{G}(p) \cap D\right) \leq 2 d(d e)^{2}$. But de $\geq 2$ implies $2 d(d e)^{2}<(d e)^{4} d$, a contradiction.

4.2. The other cases. We now handle the exceptional groups as well as ones left over from the previous subsection. We first establish a reduction result. We call hyperplanes of $G$ the hyperplanes of $V$ which are invariant subspaces of the reflections in $G$. Up to a scalar there is a unique $G$-invariant Hermitian scalar product on $V$, to which the orthogonality statement in the next proposition refers.

Proposition 4.3. Let $\phi$ be an automorphism of an irreducible reflection group $G$ which maps $R$ inside $R \cdot Z G$, where $R$ is the set of reflections of $G$. Then if either $G$ is of rank $\geq 3$ or $G$ is of rank 2 and has the property that two orthogonal hyperplanes are conjugate, then $\phi \in C \cdot A$.

Proof. The group $G$ admits a presentation by a set $S$ of distinguished reflections (i.e, reflections $s$ with eigenvalue $e^{2 i \pi / e_{s}}$, where $e_{s}$ is the order of the centralizer in $G$ of the reflecting hyperplane of $s$ ), with the order relations $s^{e_{s}}=1$ and braid relations $w=w^{\prime}$ where $w, w^{\prime} \in S^{*}$ are words of the same length; here the braid relations present the braid group of $G$ (see [Be2, theorem 0.1]). We note that two distinguished reflections are conjugate if and only if their reflecting hyperplanes are conjugate. The words $w$ and $w^{\prime}$ have the same number of elements in each class of reflections, since the braid relations present the braid group, whose abelianization is the free abelian group on the conjugacy classes of hyperplanes.

For any $s \in S$ we define a reflection $f(s)$ and an element $z(s) \in Z G$ by $\phi(s)=$ $f(s) z(s)$. When $\operatorname{dim} V \geq 3, f(s)$ and $z(s)$ are uniquely determined, but when $\operatorname{dim} V=2$ there might be two choices. We then make such an arbitrary choice, but we ask that if $s, s^{\prime} \in S$ are conjugate, i.e., there exists $g \in G$ such that $s^{\prime}={ }^{g} s$, then $z\left(s^{\prime}\right)=z(s)$, which is possible since $\phi\left(s^{\prime}\right)={ }^{\phi(g)} f(s) z(s)$ and ${ }^{\phi(g)} f(s)$ is a reflection. We now extend $f$ and $z$ to the free monoid $S^{*}$; we claim that this gives well-defined group homomorphisms from $G$. Indeed, since (obviously) for any $w \in S^{*}$ we have $\phi(w)=f(w) z(w)$, it is enough to show that $z$ induces a linear character of $G$. From the fact that each conjugacy of reflections occurs as many times in $w$ and $w^{\prime}$ it is clear that $z(w)=z\left(w^{\prime}\right)$. And from $\phi\left(s^{e_{s}}\right)=1=f(s)^{e_{s}} z(s)^{e_{s}}$, since $f(s)^{e_{s}}$ has at least one eigenvalue 1 , we conclude that $f(s)^{e_{s}}=z(s)^{e_{s}}=1$.

If $\operatorname{dim} V \geq 3$, then $f$ is an automorphism: to show that, it is enough to show that $f$ is surjective on $R$, and for that it is enough to show that $f$ is injective on $R$. If $s, s^{\prime} \in R$ and $f(s)=f\left(s^{\prime}\right)$, then $\phi\left(s s^{\prime-1}\right) \in Z G$ which implies $s s^{-1} \in Z G$ which implies $s=s^{\prime}$ since $\operatorname{dim} V \geq 3$. Thus if $\operatorname{dim} V \geq 3$, we get that $\phi$ is $f$ composed with a central automorphism (given by $s \mapsto s z\left(f^{-1}(s)\right)$ ).

There remains the case where $\operatorname{dim} V=2$ and $G$ has the property that two orthogonal hyperplanes are conjugate for which we give a more complicated argument to prove that $f$ is injective. It is enough to show that some power of $f$ is injective. Since $\phi$ and $f$ both map $Z G$ into itself, it is easy to show by induction on $r$ that for any $r$ the endomorphisms $\phi^{r}$ and $f^{r}$ still differ by a linear character with values in $Z G$. Since $\phi$ is an automorphism, it is of finite order; taking for $r$ this order, and replacing $f$ by $f^{r}$, we see that we may assume $\phi=$ Id.

We thus have $f(g)=g z(g)$ for some $Z G$-valued linear character $z$, and we want to see that $f$ is injective. We will show that $f^{2}=$ Id. It is enough to show that for a reflection $r$. We have two cases to consider: if $f(r)=r$, then $f^{2}(r)=r$. Otherwise, we must have $z(r)=\zeta^{-1} \mathrm{Id}$ where $\zeta$ is the non-trivial eigenvalue of 
$r$, and if $\langle$,$\rangle is a G$-invariant Hermitian scalar product on $V, f(r)$ is a reflection with non-trivial eigenvalue $\zeta^{-1}$ and hyperplane orthogonal to that of $r$. Since two such hyperplanes are conjugate and $f(r)^{-1}$ has the same eigenvalue as $r$, they are conjugate. Then $z(f(r))=z(r)^{-1}$ thus $f(f(r))=f(r) z(f(r))=r z(r) z(r)^{-1}=r$ and we are done.

By enumerating the hyperplanes in CHEVIE (see CHEVIE), it is easy to see that orthogonal hyperplanes are conjugate for all 2-dimensional exceptional groups except $G_{5}$ and $G_{7}$.

The preceding proposition is sufficient to handle the exceptional groups $G_{i}$ for $i \geq 12$. Indeed, an easy computer check shows that any conjugacy class $c$ such that there exists a conjugacy class $c^{\prime}$ satisfying

- $\# c^{\prime}=\# c$,

- $c^{\prime}$ contains a reflection $s^{\prime}$,

- $c$ contains an element with the same order as $s^{\prime}$,

contains the product of a reflection by an element of the center.

Finally, for the groups $G_{4}$ to $G_{12}$, as well as for the exceptions in the infinite series $G(2,1,2), G(4,2,2), G(3,3,3)$ and $G(2,2,4)$ we use the techniques of section [6 to determine all automorphisms and to check that they are the product of an element of $C$ and an automorphism which preserves the reflections.

\section{REFLECTION REPRESENTATIONS}

By a reflection representation of a reflection group $G$, we mean a faithful representation such that the image of a reflection of $G$ is a reflection. The corresponding characters are called reflection characters. Here we prove Theorem 1.2, whose statement we recall.

Theorem 5.1. Let $G \subset \mathrm{GL}(V)$ be an irreducible complex reflection group, where $V$ is a vector space over the field of definition $K$ of $G$. Then the reflection characters of $G$ are the transforms of $\chi_{V}$ by the action of $\operatorname{Gal}(K / \mathbb{Q})$.

Before going on with the proof, we remark that this proves indeed that any automorphism which preserves the reflections is a Galois automorphism attached to some $\gamma \in \operatorname{Gal}(K / \mathbb{Q})$, since it sends $\chi_{V}$ to some $\gamma\left(\chi_{V}\right)$.

Proof of Theorem 5.1. The theorem is proved by a computer check for the exceptional reflection groups, by looking at the character tables. Both the action of the Galois group, and which representations are faithful and send reflections to reflections are readily seen from the character tables.

We now look at the groups $G(d e, e, r)$. We first look at the case $e=1$. The result is clear if $G$ is cyclic so we assume $r>1$, and use the notations of section 2.3 ,

We first describe the action of $\operatorname{Gal}(K / \mathbb{Q})$ on the representations of $G(d, 1, r)$.

Lemma 5.2. For $i \in \mathbb{Z}$ prime to $d$, let $\mathfrak{s}_{i} \in \operatorname{Gal}\left(\mathbb{Q}\left(\zeta_{d}\right) / \mathbb{Q}\right)$ be the element defined by $\mathfrak{s}_{i}\left(\zeta_{d}\right)=\zeta_{d}^{i}$. Then the partition tuple $\mathfrak{s}_{i}(\boldsymbol{\lambda})$ such that $\mathfrak{s}_{i} \circ \rho_{\boldsymbol{\lambda}} \simeq \rho_{\mathfrak{s}_{i}(\boldsymbol{\lambda})}$ is given by $\mathfrak{s}_{i}(\boldsymbol{\lambda})=\left(\lambda_{0}, \lambda_{i}, \lambda_{2 i}, \ldots, \lambda_{(d-1) i}\right)$ (where the indices are taken $(\bmod d)$ ).

Proof. This is immediate using the model given in 2.3. The action of $\mathfrak{s}_{i}$ is trivial on $\rho_{\boldsymbol{\lambda}}\left(s_{i}\right)$ and maps $\rho_{\boldsymbol{\lambda}}(t)$ to $\rho_{\boldsymbol{\lambda}}(t)^{i}$, from which the result follows.

We now determine which $\rho_{\boldsymbol{\lambda}}$ are faithful reflection representations. 
Lemma 5.3. The faithful representations $\rho_{\boldsymbol{\lambda}}$ of $G(d, 1, r), r>1$ such that $\rho_{\boldsymbol{\lambda}}(t)$ is a reflection are the $\rho_{\boldsymbol{\lambda}^{(i)}}$ for $i$ prime to $d$, where $\lambda_{0}^{(i)}=\{r-1\}$ or $\lambda_{0}^{(i)}=\left\{1^{r-1}\right\}$, and $\lambda_{i}^{(i)}=\{1\}$.

Proof. First, notice that there is only one standard tableau corresponding to a given Young diagram and with a given content if and only if the diagram is a column or a row. For a partition $\lambda$ we denote by $|\lambda|$ the size of $\lambda$ (the sum of the lengths of its parts), and call the height of $\lambda$ the number of its parts, namely the number of rows in the corresponding diagram. Since $\mathbf{T} \in \mathcal{T}(\boldsymbol{\lambda})$ are eigenvectors of $\rho_{\boldsymbol{\lambda}}(t)$ with eigenvalue $\zeta_{d}^{\mathbf{T}(1)}$, there must be exactly one $\mathbf{T} \in \mathcal{T}(\boldsymbol{\lambda})$ such that $\mathbf{T}(1) \neq 0$. This means that there must be exactly one $i \neq 0$ such that $\left|\lambda_{i}\right| \neq 0$, with a Young diagram a line or a column. We must also have that $\lambda_{0}$ is a line or a column; if $\left|\lambda_{0}\right|=0$, then the representation is of dimension 1 and cannot be faithful since $r>1$. Also if $\left|\lambda_{i}\right|>1$, then $r \geq 3$ and there are at least two $\mathbf{T}$ such that $\mathbf{T}(1)=i$. So we must have $\lambda_{i}=\{1\}$. Finally, note that $i$ must be prime to $d$, otherwise $\rho_{\boldsymbol{\lambda}}$ is not faithful (since $\rho_{\boldsymbol{\lambda}}(t)$ does not have order $d$ ). We thus get exactly the cases in the statement.

Lemma 5.4. The representations $\rho_{\boldsymbol{\lambda}}$ of $G(d, 1, r)$ such that $\rho_{\boldsymbol{\lambda}}\left(s_{i}\right)$ is a reflection are those such that there exists $i \neq j$ such that $\lambda_{i}=\{r-1\}$ and $\lambda_{j}=\{1\}$, or there exists only one $i$ with $\left|\lambda_{i}\right| \neq 0$, and then either $\lambda_{i}=\{r-1,1\}$ or $\lambda_{i}=\{2,2\}$ (this last case for $r=4$ ).

Proof. The formula (2.2) shows that $\rho_{\boldsymbol{\lambda}}\left(s_{i}\right)$ in the basis $\mathcal{T}(\boldsymbol{\lambda})$ is block-diagonal, with diagonal blocks indexed by the basis elements $\mathbf{T}$ and $\mathbf{T}_{i \leftrightarrow i+1}$, which are thus of size 1 when $\mathbf{T}_{i \leftrightarrow i+1}=0$ and of size 2 otherwise.

(i) If $\mathbf{T}_{i \leftrightarrow i+1} \neq 0$ the corresponding block is $\left(\begin{array}{cc}\frac{1}{a(i, i+1)} & 1+\frac{1}{a(i, i+1)} \\ 1-\frac{1}{a(i, i+1)} & \frac{-1}{a(i, i+1)}\end{array}\right)$ whose eigenvalues are 1 and -1 .

(ii) If $\mathbf{T}_{i \leftrightarrow i+1}=0$, then $i$ and $i+1$ are in the same tableau, and in the same line or column. We have that $\mathbf{T}$ is an eigenvector of $\rho_{\boldsymbol{\lambda}}\left(s_{i}\right)$, for the eigenvalue $\frac{1}{a(i, i+1)}=1$ when $i$ and $i+1$ are in the same line and $\frac{1}{a(i, i+1)}=-1$ when $i$ and $i+1$ are in the same column.

For $\rho_{\boldsymbol{\lambda}}\left(s_{i}\right)$ to be a reflection there cannot be more than two $j$ with $\left|\lambda_{j}\right| \neq 0$, otherwise case (i) would occur more than once (with $i$ and $i+1$ in different tableaux). Similarly, if there are two $j$ such that $\left|\lambda_{j}\right| \neq 0$, then the two Young diagrams must be lines or columns, otherwise case (i) would occur more than once. Actually, the two diagrams must be lines since if one is a column of height $>1$, then case (ii) with eigenvalue -1 has to occur at least once, and for $\rho_{\boldsymbol{\lambda}}\left(s_{r-1}\right)$ to be a reflection one of the lines has to be of length 1, otherwise there would exist at least two different $\mathbf{T}$ with $i$ in the first tableau and $i+1$ in the second, which leads to too many instances of (i).

We now look at the case when only one $\left|\lambda_{j}\right| \neq 0$. The diagram must not be a line or a column, otherwise $\rho$ is not faithful. For $i=r-1$ case (i) will occur more than once if the diagram has more than two corners. If there are two corners, when they are removed the rest must be a line or a column; this means the diagram must be a hook; and the height of the hook has to be 2 , otherwise case (ii) with eigenvalue -1 will occur. Finally, if there is one corner the diagram is a rectangle; this rectangle has to be $2 \times 2$, otherwise by removing the corner one would get a diagram with 
two corners which is not a hook which would lead to too many eigenvalues -1 for $s_{r-2}$.

We are left exactly with the cases in the statement of the lemma.

From the two previous lemmas we get the following.

Corollary 5.5. The faithful reflection representations of $G(d, 1, r)$ are the $\rho_{\boldsymbol{\lambda}^{(i)}}$ for $i$ prime to $d$, where $\lambda_{0}^{(i)}=\{r-1\}$ and $\lambda_{i}^{(i)}=\{1\}$.

The reflection representation of $G(d, 1, r)$ is $\rho_{\boldsymbol{\lambda}^{(1)}}$ and by Lemma 5.2 we have $\mathfrak{s}_{i} \circ \rho_{\boldsymbol{\lambda}^{(1)}}=\rho_{\boldsymbol{\lambda}^{(i)}}$ which together with Corollary 5.5 proves the theorem for $G(d, 1, r)$.

Let us now look at $G(d e, e, r)$.

Lemma 5.6. The faithful irreducible reflection representations of $G(d e, e, r)$ are the restrictions to $G(d e, e, r)$ of the faithful irreducible reflection representations of $G(d e, 1, r)$.

Proof. Let $\rho_{0}$ be a faithful irreducible reflection representation of $G(d e, e, r)$ and $\rho$ an irreducible representation of $G(d e, 1, r)$ whose restriction contains $\rho_{0}$. We first assume by contradiction that the restriction of $\rho$ to $G(d e, e, r)$ is not irreducible (i.e., distinct from $\rho_{0}$ ). This implies $r \geq 3$, as otherwise $\operatorname{dim} \rho \leq 2$ hence $\operatorname{dim} \rho_{0} \leq 1$, contradicting the faithfulness of $\rho_{0}$ since $G(d e, e, r)$ is not abelian. Since $\rho_{0}\left(s_{1}\right)$ is a reflection, the restriction of $\rho_{0}$ to $G(d e, e, r-1)$ contains exactly one component $\varphi$ such that $\varphi_{1}\left(s_{1}\right)$ is a reflection. From [M2, proposition 3.1] we get from our assumption of non-irreducibility of $\operatorname{Res}_{G(d e, e, r)}^{G(d e, 1, r)} \rho$ that $\varphi_{1}$ is the restriction to $G(d e, e, r-1)$ of an irreducible representation $\tilde{\varphi}$ of $G(d e, e, r-1)$. Then $\tilde{\varphi}_{1}\left(s_{1}\right)$ is a reflection, hence $\tilde{\varphi}$ corresponds to a multipartition $\boldsymbol{\mu}$ of the form given by Lemma 5.4. Let $\boldsymbol{\lambda}$ be the multipartition corresponding to $\rho$. Since $\tilde{\varphi}$ embeds into the restriction of $\rho$ to $G(d e, 1, r-1), \boldsymbol{\lambda}$ is obtained by adding one box to $\boldsymbol{\mu}$ (see Ze, p. 104] for this branching rule). The only possibility for the restriction of $\rho$ to $G(d e, e, r)$ not to be irreducible is then for $r=3$, de $=3 k$ and for some $i$ we have $\lambda_{i+2 k}=\lambda_{i+k}=\lambda_{i}=\{1\}$, the remaining parts of $\boldsymbol{\lambda}$ being empty. In that case we readily check that $\rho$ hence $\rho_{0}$ is not faithful on $G(d e, e, r)$; for instance, it can be deduced from [Ze, pp. 93-106] that the central character of $\rho_{\boldsymbol{\lambda}}$ is given by $\zeta_{d e} \mapsto \zeta_{d e}^{\sum_{j=0}^{d e-1} j\left|\lambda_{j}\right|}$, which in our case is equal to $\zeta_{3 k}^{3 i+3 k}=\zeta_{k}^{i}$, hence $\zeta_{3} \in G(3 k, 3 k, 3)$ acts by $1=\left(\zeta_{k}\right)^{k}$, contradicting faithfulness. This shows that $\rho_{0}$ is the restriction of an irreducible representation of $G(d e, e, r)$, which we assume from now on.

We use the analysis of when $\rho_{\boldsymbol{\lambda}}\left(s_{i}\right)$ is a reflection. Notice first that the representations with only one diagram are not faithful, since by formula (2.3) $s_{1}$ and $s_{1}^{t}$ have the same image since for any $\mathbf{T}$ we have $\mathbf{T}(1)=\mathbf{T}(2)$. Thus we are in the case where we have $i \neq j$ such that $\lambda_{i}=\{n-1\}$ and $\lambda_{j}=\{1\}$. Actually, for the representation to be faithful we must have $\rho_{\boldsymbol{\lambda}}\left(s_{1}^{t^{k}}\right) \neq \rho_{\boldsymbol{\lambda}}\left(s_{1}\right)$ for any $k$ such that $1 \leq k<d e$, thus $\mathbf{T}(2)-\mathbf{T}(1)$ must be prime to de, i.e., $j-i$ must be prime to de.

Analyzing when $\rho_{\boldsymbol{\lambda}}\left(t^{e}\right)$ is a reflection along the same lines as we did for $\rho_{\boldsymbol{\lambda}}(t)$, we find that $j \equiv 0(\bmod d)$. Since $\rho_{\boldsymbol{\lambda}}$ and $\rho_{\sigma(\boldsymbol{\lambda})}$ have same restriction to $G(d e, e, r)$ where $\sigma$ is the "shift" by $d$ as in section 2.4 we may assume $j=0$. Thus we have indeed the same representations as in Corollary [5.5, which proves the lemma.

The theorem for $G(d e, e, r)$ follows immediately from the above lemma since the reflection representation of $G(d e, e, r)$ is the restriction of the reflection representation of $G(d e, 1, r)$, and the Galois action commutes with the restriction. 


\section{TheOREMS 1.4 AND 1.6 FOR THE EXCEPTIONAL GROUPS}

To verify 1.6 for the exceptional reflection groups, we have used the GAP package CHEVIE (see [CHEVIE]), which contains information about them, including:

- their realization via a permutation representation (on a set of "roots" in $V)$,

- their character table.

The character tables allow us to determine the permutation of $\operatorname{Irr}(G)$ effected by an element of $\operatorname{Gal}(K / \mathbb{Q})$. The method we have used to check 1.4 is to compute the group of outer automorphisms of $G$ which preserve the set of reflections, and then to find a subgroup which induces the same permutation group on $\operatorname{Irr}(G)$ as $\operatorname{Gal}(K / \mathbb{Q})$. We recall from Proposition 3.1 that an element of Out $(G)$ is completely determined by the permutation it induces on $\operatorname{Irr}(G)$.

To compute automorphisms, we use a presentation for $G$, by means of a diagram (see $[\mathrm{BMR}$ and $[\mathrm{BM}$ ); if $S$ is the generating set for this presentation, to enumerate the automorphisms which preserve the reflections, we enumerate up to inner automorphisms all tuples of reflections of $G$ which satisfy the relations for $G$, and generate $G$.

If we want to enumerate all automorphisms, we have to extend the search to other conjugacy classes which have the same size and same order as the conjugacy class of a reflection. We only need to compute the whole $\operatorname{group} \operatorname{Aut}(G)$ for 2dimensional groups, to meet the requirements at the end of section 4 , and for these groups the process above is quite fast.

To find directly the orbits of $G$ on the tuples of reflections is a computation too large for the larger groups, such as $G_{34}$ which has about $4.10^{7}$ elements; thus we use a recursive process: we choose the image of the first generator $s$ (which amounts to choosing a representative for each orbit of $G$ on the reflections); then we find the $C_{G}(s)$-orbits of tuples which satisfy the relations for $S-\{s\}$ (by a recursive process) and then select those that have the required relations with $s$; and finally weed out the tuples which do not generate $G$. This allows us to compute $\bar{A}$ in at most a few hours even for the largest groups.

To find the image of $\bar{\iota}_{\chi}(\Gamma)$ in $\bar{A}$ is rather easy since for the exceptional groups the morphism $\Gamma \stackrel{\bar{\iota}}{\rightarrow} \bar{A}$ is either surjective or has an image of index 2 (the last occurs exactly for $G_{5}, G_{7}$ and $G_{28}=F_{4}$, which, in agreement with Theorem 1.3. are also the only exceptional groups where $\bar{N} \neq 1$; in these cases, with the notation of Table $1 . \bar{N}$ induces respectively the automorphism $(s, t) \mapsto(t, s)$, the automorphism $(s, t, u) \mapsto\left(s, u^{-1}, t^{-1}\right)$, and the diagram automorphism of $\left.F_{4}\right)$.

To check 1.6, we must lift $\bar{\iota}$ to the group $\operatorname{Aut}(G)$, that is, find representatives in $\operatorname{Aut}(G)$ of elements of the image of $\bar{\iota}$ which satisfy the relations for $\Gamma$. Since for exceptional groups $\Gamma$ is an abelian 2-group, these relations are order relations, plus commutation relations.

Few representatives of a given automorphism turn out to have the right order, so we first compute all representatives which have the right order, and then it is an easy job to choose among them elements which commute (this procedure of course fails for $G_{27}$ for which we must construct $\tilde{\iota}$ rather than $\iota$; in this case we take $K^{\prime}=\mathbb{Q}\left(\zeta_{15}\right)$; the group $\operatorname{Gal}\left(K^{\prime} / \mathbb{Q}\right)$ is generated by $\mathfrak{c}$ and $\mathfrak{s}_{7}$, this last element being of order 4 (it is of order 2 in $\operatorname{Gal}(K / \mathbb{Q})$ ); we can map these to automorphisms of 
order 2 and 4 respectively commuting to each other — but it is impossible to map $\mathfrak{s}_{7}$ to an automorphism of order 2).

The only computational problem in this procedure is to compute the product of two automorphisms; since an automorphism $\varphi$ is represented by the image $\varphi(S)$, to do this we need an expression as words in $S^{*}$ of the elements of $\varphi(S)$. For all groups but $G_{34}$ this was solved by enumerating all elements of $G$ by standard permutation group algorithms using a base and a strong generating set. For $G_{34}$ we managed by only considering automorphisms which extend the one for $G_{33}$; since the normalizer of $G_{33}$ in $G_{34}$ is $G_{33}$ times the center of $G_{34}$, such automorphisms map the last generator of $G_{34}$ to a conjugate by $G_{33}$, which makes the computation feasible.

Table 1 summarizes our results for the exceptional reflection groups; it gives the diagram for $G$, and then gives the value of $K$ and describes $\iota$ in terms of the generators $S$ given by the diagram.

The nodes of the diagrams are labeled by the elements of $S$; we give to these elements names in the list $s, t, u, v, w, x$ in that order. A number inside the node gives the order of the corresponding reflection when it is not 2 . To express the braid relations, we use the conventions for Coxeter diagrams: two nodes which are not connected correspond to commuting reflections. A single line connecting two nodes $s$ and $t$ corresponds to the relation $s t s=t s t$, a double line to stst $=t s t s$, a triple line to ststst $=t s t s t s$, and a line labeled with the number $n$ corresponds to a braid relation $\underbrace{s t s t \cdots}_{n \text { terms }}=\underbrace{t s t s \cdots}_{n \text { terms }}$.

These conventions are extended by additional ones; a circle joining 3 nodes, $s$, $t$ and $u$, with the number $n$ inside (when there is no number inside, 3 is to be understood) corresponds to a clockwise circular relation $\underbrace{\text { stust } \cdots}_{n \text { terms }}=\underbrace{\text { tustu } \cdots}_{n \text { terms }}=$ $\underbrace{\text { ustus } \cdots ;}$ for instance, the relation for $G_{7}, G_{11}, G_{19}$ and the generators $s, t, u$ of $n$ terms

$G_{31}$ is $s t u=t u s=u s t$. Similarly, the 6 inside the triangle for $G_{33}$ and $G_{34}$ means that in addition to length 3 braid relations implied by the sides of the triangle, there is the circular relation tuvtuv $=u v t u v t=v t u v t u$.

The double line in $G_{29}$ expresses a length 4 braid relation between $v$ and $u t$ : vutvut $=$ utvutv. Let us mention that the exceptional characters of $G_{29}$ are the 6-dimensional rational ones which appear in the tenth symmetric power $S^{10}(V)$.

The triangle symbol for $G_{24}$ and $G_{27}$ corresponds to the relation $s(u t s)^{2}=$ $(s t u)^{2} t$. Finally, the groups $G_{13}$ and $G_{15}$ have more complicated relations which are spelled out below the diagram.

The groups $G_{28}=F_{4}, G_{35}=E_{6}, G_{36}=E_{7}$ and $G_{37}=E_{8}$, which are rational, do not appear in the table since our theorems are trivial for them.

The map $\iota$ is specified by giving for generators $\gamma$ of $\Gamma$ the tuple $\iota(\gamma)(S)$ (where $S$ is in the order $s, t, u, v, w, x)$. The elements of $\Gamma$ are denoted by $\mathfrak{c}$ for complex conjugation and by $\mathfrak{s}_{i}$ for the following element: let $\mathbb{Q}\left(\zeta_{n}\right)$ be the smallest cyclotomic field containing $K$; then $\mathfrak{s}_{i}$ is the restriction to $K$ of the Galois automorphism of $\mathbb{Q}\left(\zeta_{n}\right)$ which sends $\zeta_{n}$ to $\zeta_{n}^{i}$. 
Table 1: The morphism $\tilde{\iota}$

\begin{tabular}{|c|c|c|c|}
\hline Group & Diagram & Field & $\begin{array}{l}\tilde{\imath} \\
\end{array}$ \\
\hline$G_{4}$ & ${ }_{s}^{(3)}-{ }_{t}^{(3)}$ & $\mathbb{Q}\left(\zeta_{3}\right)$ & $\mathfrak{c} \mapsto\left(s^{-1},{ }^{s} t^{-1}\right)$ \\
\hline$G_{5}$ & ${ }_{s}^{(3)}={ }_{t}^{3}$ & $\mathbb{Q}\left(\zeta_{3}\right)$ & $\mathfrak{c} \mapsto\left(s^{-1},{ }^{s} t^{-1}\right)$ \\
\hline$G_{6}$ & & $\mathbb{Q}\left(\zeta_{12}\right)$ & $\begin{array}{l}\mathfrak{c} \mapsto\left(s^{-1}, t^{-1}\right), \\
\mathfrak{s}_{7} \mapsto\left(s^{t^{-1}} s t, t\right)\end{array}$ \\
\hline$G_{7}$ & & $\mathbb{Q}\left(\zeta_{12}\right)$ & $\begin{array}{c}\mathfrak{c} \mapsto\left(s^{-1}, t^{-1},\left(u^{-1}\right)^{s}\right), \\
\mathfrak{s}_{7} \mapsto\left(s^{u t}, t, u\right)\end{array}$ \\
\hline$G_{8}$ & & $\mathbb{Q}(\mathrm{i})$ & $\mathfrak{c} \mapsto\left(s^{-1}, t^{-1}\right)$ \\
\hline$G_{9}$ & & $\mathbb{Q}\left(\zeta_{8}\right)$ & $\begin{array}{l}\mathfrak{c} \mapsto\left(s^{-1}, t^{-1}\right), \\
\quad \mathfrak{s}_{5} \mapsto\left(s^{t t s}, t\right)\end{array}$ \\
\hline$G_{10}$ & & $\mathbb{Q}\left(\zeta_{12}\right)$ & $\begin{aligned} \mathfrak{c} \mapsto & \left(\left(s^{-1}\right)^{t^{-1}}, t^{-1}\right) \\
\quad \mathfrak{s}_{7} & \mapsto\left(s^{t t s s}, t^{-1}\right)\end{aligned}$ \\
\hline$G_{11}$ & & $\mathbb{Q}\left(\zeta_{24}\right)$ & $\begin{array}{c}\mathfrak{c} \mapsto\left(s^{-1}, t^{-1},\left(u^{-1}\right)^{s}\right), \\
\mathfrak{s}_{13} \mapsto\left(s^{t s u t}, t, u\right), \\
\mathfrak{s}_{19} \mapsto\left(s, t,\left(u^{-1}\right)^{t u^{-1} s}\right)\end{array}$ \\
\hline$G_{12}$ & & $\mathbb{Q}(\sqrt{-2})$ & $\mathfrak{c} \mapsto(t, s, u)$ \\
\hline$G_{13}$ & $\begin{array}{c}s(5) u \\
\text { tust }=u s t u, \\
\text { stust }=\text { ustus }\end{array}$ & $\mathbb{Q}\left(\zeta_{8}\right)$ & $\begin{array}{c}\mathfrak{c} \mapsto\left(s, u, t^{s}\right), \\
\mathfrak{s}_{3} \mapsto\left(s, u^{t u}, u^{s u}\right)\end{array}$ \\
\hline$G_{14}$ & $\bigcirc_{s}-8$ & $\mathbb{Q}\left(\zeta_{3}, \sqrt{-2}\right)$ & $\begin{aligned} \mathfrak{c} & \mapsto\left(s^{-1},\left(t^{-1}\right)^{s}\right), \\
& \mathfrak{s}_{7} \mapsto\left(s, t^{\text {sttst }}\right)\end{aligned}$ \\
\hline$G_{15}$ & $\begin{aligned} s t u & =u s t \\
\text { tusts } & =\text { ustst }\end{aligned}$ & $\mathbb{Q}\left(\zeta_{24}\right)$ & $\begin{aligned} \mathfrak{c} & \mapsto\left(s, t^{-1}, u^{s}\right), \\
\mathfrak{s}_{13} & \mapsto\left(s^{u s u}, t, u\right), \\
\mathfrak{s}_{19} & \mapsto\left(s, t, u^{s u s}\right)\end{aligned}$ \\
\hline
\end{tabular}




\begin{tabular}{|c|c|c|c|}
\hline Group & Diagram & Field & 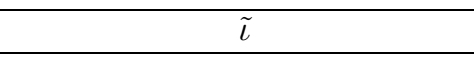 \\
\hline$G_{16}$ & $\underset{s}{(5)}-{ }_{t}$ & $\mathbb{Q}\left(\zeta_{5}\right)$ & $\mathfrak{s}_{2} \mapsto\left(s^{2},\left(t^{2}\right)^{s^{2} t^{-1} s}\right)$ \\
\hline$G_{17}$ & $\mathrm{O}_{s} \equiv{ }_{t}$ & $\mathbb{Q}\left(\zeta_{20}\right)$ & $\begin{aligned} \mathfrak{c} & \mapsto\left(s^{-1}, t^{-1}\right), \\
\mathfrak{s}_{7} & \mapsto\left(s^{t^{-1}} s t^{2}, t^{2}\right)\end{aligned}$ \\
\hline$G_{18}$ & & $\mathbb{Q}\left(\zeta_{15}\right)$ & $\begin{aligned} \mathfrak{c} \mapsto\left(s^{-1}, t^{-1}\right), \\
\mathfrak{s}_{7} \mapsto\left(s^{t^{-1}}, t^{2}\right)\end{aligned}$ \\
\hline$G_{19}$ & & $\mathbb{Q}\left(\zeta_{60}\right)$ & $\begin{aligned} \mathfrak{c} & \mapsto\left(s^{t}, t^{-1}, u^{-1}\right), \\
\mathfrak{s}_{7} & \mapsto\left(s^{t s}, t^{s t^{-1}}, u^{2}\right), \\
\mathfrak{s}_{41} & \mapsto\left(s,\left(t^{-1}\right)^{(u t)^{s u}}, u\right)\end{aligned}$ \\
\hline$G_{20}$ & (3) 5 & $\mathbb{Q}\left(\zeta_{3}, \sqrt{5}\right)$ & $\begin{aligned} \mathfrak{c} & \mapsto\left(s^{-1}, t^{-1}\right), \\
\mathfrak{s}_{7} & \mapsto\left(s, s^{\left(t s^{-1}\right)^{2}}\right)\end{aligned}$ \\
\hline$G_{21}$ & & $\mathbb{Q}\left(\zeta_{12}, \sqrt{5}\right)$ & 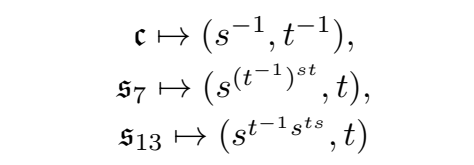 \\
\hline$G_{22}$ & & $\mathbb{Q}(\mathrm{i}, \sqrt{5})$ & $\begin{array}{c}\mathfrak{c} \mapsto(u, t, s), \\
\mathfrak{s}_{7} \mapsto\left(u^{s t}, u^{t s}, t^{u s}\right)\end{array}$ \\
\hline$G_{23}=H_{3}$ & & $\mathbb{Q}(\sqrt{5})$ & $\mathfrak{s}_{2} \mapsto\left(u^{t s t s}, u, t\right)$ \\
\hline$G_{24}$ & & $\mathbb{Q}(\sqrt{-7})$ & $\mathfrak{c} \mapsto(u, t, s)$ \\
\hline$G_{25}$ & (3)- ${ }_{s}(3)-{ }_{u}$ & $\mathbb{Q}\left(\zeta_{3}\right)$ & $\mathfrak{c} \mapsto\left(s^{-1}, t^{-1}, u^{-1}\right)$ \\
\hline$G_{26}$ & & $\mathbb{Q}\left(\zeta_{3}\right)$ & $\mathfrak{c} \mapsto\left(s^{-1}, t^{-1}, u^{-1}\right)$ \\
\hline$G_{27}$ & & $\begin{array}{c}\mathbb{Q}\left(\zeta_{3}, \sqrt{5}\right) \\
K^{\prime}=\mathbb{Q}\left(\zeta_{15}\right)\end{array}$ & $\begin{aligned} \mathfrak{c} \mapsto\left(s^{t u t}, t, u\right), \\
\mathfrak{s}_{7} \mapsto\left(u^{t s}, t^{u}, t\right)\end{aligned}$ \\
\hline$G_{29}$ & & $\mathbb{Q}(\mathrm{i})$ & $\mathfrak{c} \mapsto\left(s, t, u, v^{u}\right)$ \\
\hline$G_{30}=H_{4}$ & & $\mathbb{Q}(\sqrt{5})$ & $\mathfrak{s}_{2} \mapsto\left(u^{t s t s}, u, t, v^{\text {utstuststuvuts }}\right)$ \\
\hline
\end{tabular}




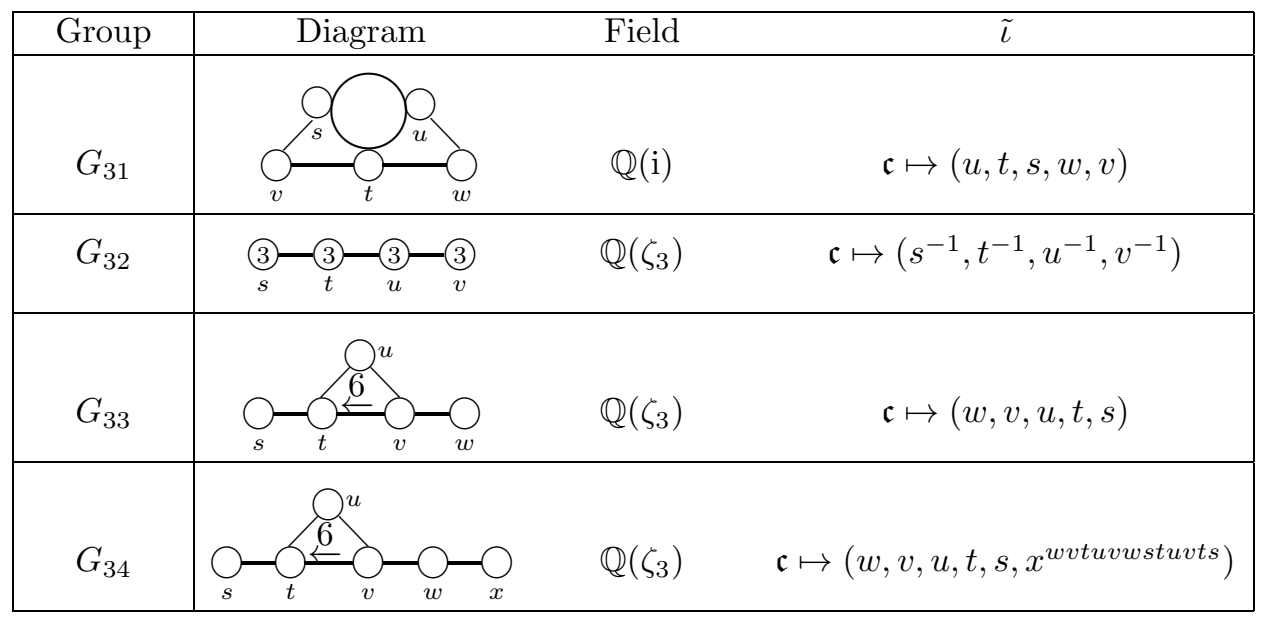

We have some observations to make on Table 1. First, the morphism $\tilde{\iota}$ is not unique; often, there are even many different orbits of possible $\tilde{\iota}$ up to inner automorphisms of $G$. Each time we have chosen one that preserves a parabolic subgroup, which will be used to construct nice models of the reflection representation of $G$ in section 9 ,

This is not always the choice with the simplest formulas. In particular, for Shephard groups (those which have the same diagram, apart from the order of the reflections, as a Coxeter group, thus the same braid group as a Coxeter group), it turns out that it is always possible to find a morphism which maps the complex conjugation $\mathfrak{c}$ to the automorphism which sends each element of $S$ to its inverse. When $\mathfrak{c}$ does not generate $\operatorname{Gal}(K / \mathbb{Q})$, one can send $\mathfrak{s}_{7}$ to $\left(s^{t s^{-1}}, t^{-1}\right)$ for $G_{10}$ and $\mathfrak{s}_{2}$ to $\left(s^{2},\left(s^{2}\right)^{t^{-2} s^{2}}\right)$ for $G_{16}$ to get such a morphism (in the case of $G_{14}, \mathfrak{s}_{7}$ can keep the same image as in Table 1). A general explanation for this phenomenon would be desirable.

\section{Theorems 1.4, 1.6 AND 1.7 FOR THE INFINITE SERIES}

The field of the definition of $G(d e, e, r)$ is $K=\mathbb{Q}\left(\zeta_{d e}\right)$, except when $d=1$ and $r=2$. In the last case, $G(e, e, 2)$ is the dihedral group of order $2 e$, for which $K=\mathbb{Q}\left(\cos \left(\frac{2 \pi}{e}\right)\right)=\mathbb{Q}\left(\zeta_{e}+\zeta_{e}^{-1}\right)$ and we set $K^{\prime}=\mathbb{Q}\left(\zeta_{e}\right)$.

In any case the natural representation of $G(d e, e, r)$ via monomial matrices is over the vector space $V=K^{\prime r}$, and writing $V=V_{0} \otimes_{\mathbb{Q}} K^{\prime}$ where $V_{0}$ is defined by the standard basis of $V$, the set $G(d e, e, r)$ is globally invariant under the induced action of $\operatorname{Gal}\left(K^{\prime} / \mathbb{Q}\right)$ on $\mathrm{GL}_{r}\left(K^{\prime}\right)$. It follows that this action induces a morphism $\eta: \operatorname{Gal}\left(\mathbb{Q}\left(\zeta_{d e}\right) / \mathbb{Q}\right) \rightarrow \operatorname{Aut}(G)$. The purpose of this section is to show that a slight variation on $\eta$ satisfies the properties of Theorem [1.6 which implies the weaker Theorem 1.4. Actually, we will get a more precise version of Theorem 1.6] stating the $\tilde{\iota}_{\chi}$ equivariance of some models, which also gives Theorem 1.7.

7.1. Dihedral groups. In this section we write $s^{\prime}=s_{1}^{\prime}, s=s_{1}$, thus the dihedral group $G(e, e, 2)$ is generated by $S=\left\{s^{\prime}, s\right\}$ where

$$
s^{\prime}=\left(\begin{array}{cc}
0 & \zeta_{e} \\
\zeta_{e}^{-1} & 0
\end{array}\right), \quad s=\left(\begin{array}{cc}
0 & 1 \\
1 & 0
\end{array}\right)
$$


with relations $s^{2}=s^{\prime 2}=1,\left(s^{\prime} s\right)^{e}=1$. For $i$ prime to $e$ we have $\eta\left(\mathfrak{s}_{i}\right)(s)=s$ and $\eta\left(\mathfrak{s}_{i}\right)\left(s^{\prime}\right)=\left(s^{\prime} s\right)^{i} s$. In particular, $\eta\left(\mathfrak{s}_{i}\right)\left(s^{\prime} s\right)=\left(s^{\prime} s\right)^{i}$. We may also check that for any $x \in G$ we have $\eta\left(\mathfrak{s}_{-i}\right)(x)=s \eta\left(\mathfrak{s}_{i}\right)(x) s$.

Recall that $K=\mathbb{Q}\left(\zeta_{e}+\zeta_{e}^{-1}\right)$ and let $K^{\prime}=\mathbb{Q}\left(\zeta_{e}\right)$. It follows that the quotient $\Gamma^{\prime}=\operatorname{Gal}\left(K^{\prime} / \mathbb{Q}\right) \rightarrow \Gamma=\operatorname{Gal}(K / \mathbb{Q})$ is obtained by identifying $\mathfrak{s}_{i}$ and $\mathfrak{s}_{-i}$, thus the above computations show that the composed morphism $\Gamma^{\prime} \stackrel{\eta}{\rightarrow} \operatorname{Aut}(G) \rightarrow \operatorname{Out}(G)$ factors through $\Gamma$, leading to the following commutative diagram where we have written $\tilde{\iota}$ for $\eta$.

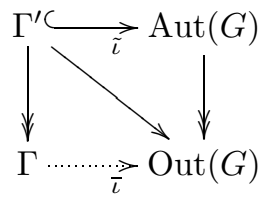

The maps $\tilde{\iota}$ and $\bar{\iota}$ above satisfy the hypotheses of Theorems 1.4 and 1.6

We note that the above reflection representation is actually $\tilde{\iota}$-equivariant, thus we have Theorem 1.7 for the reflection representation.

It is clear that Theorems 1.4, 1.6] and 1.7 hold for the linear characters of $G$, because they take values in $\mathbb{Q}$. The remaining irreducible representations of $G$ have a model of the form

$$
s^{\prime} \mapsto\left(\begin{array}{cc}
0 & \zeta_{e}^{r} \\
\zeta_{e}^{-r} & 0
\end{array}\right), \quad s \mapsto\left(\begin{array}{cc}
0 & 1 \\
1 & 0
\end{array}\right), \quad s^{\prime} s \mapsto\left(\begin{array}{cc}
\zeta_{e}^{r} & 0 \\
0 & \zeta_{e}^{-r}
\end{array}\right)
$$

for some $r$, and are determined up to isomorphism by the value of $\zeta_{e}^{r}+\zeta_{e}^{-r}$, so Theorems 1.4, 1.6 and 1.7 also hold for them.

Construction of $\iota$. We will now determine when it is possible to take $K^{\prime}=K$. It is classical that the short exact sequence

$$
1 \rightarrow \operatorname{Gal}\left(\mathbb{Q}\left(\zeta_{e}\right) / \mathbb{Q}\left(\zeta_{e}+\zeta_{e}^{-1}\right)\right) \rightarrow \operatorname{Gal}\left(\mathbb{Q}\left(\zeta_{e}\right) / \mathbb{Q}\right) \rightarrow \operatorname{Gal}\left(\mathbb{Q}\left(\zeta_{e}+\zeta_{e}^{-1}\right) / \mathbb{Q}\right) \rightarrow 1
$$

splits precisely when $\mathbb{Q}\left(\zeta_{e}\right)$ contains a quadratic imaginary extension of $\mathbb{Q}$. This is the case if and only if $4 \mid e$ or $e$ has a prime factor congruent to 3 modulo 4 . In that case one can define injective morphisms $\Gamma \rightarrow \operatorname{Aut}(G)$ by composition:

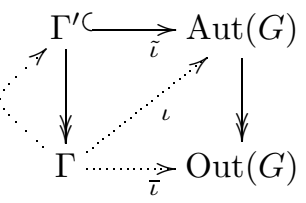

We cannot expect to lift this morphism to $\operatorname{Aut}(G)$ in general, as already shown in the example $e=5$. In that case, it is easily checked that $\Gamma=\operatorname{Gal}(\mathbb{Q}(\sqrt{5}) / \mathbb{Q})$ has order two and that all involutive automorphisms of $G$ are inner.

If the exact sequence splits, we let $H<\Gamma^{\prime}$ be a section of $\Gamma$. Since $H$ has index two in $\Gamma^{\prime}$ we know that $H$ is normal in $\Gamma^{\prime}$ and, by elementary Galois theory, we deduce from $K=K^{\prime} \cap \mathbb{R}$ that $H=\operatorname{Gal}\left(K^{\prime} / \mathbb{Q}(\mathrm{i} \alpha)\right)$ for some quadratic number $\alpha \in \mathbb{R}$. Let $\rho: G \rightarrow \mathrm{GL}_{2}\left(K^{\prime}\right)$ denote an arbitrary 2-dimensional irreducible representation of $G$, following the models described above. An intertwiner between $\rho$ and $\mathfrak{c} \circ \rho$ is given by $\rho(s) \in \mathrm{GL}_{2}(\mathbb{Q})$. Let

$$
M=\frac{1}{4 \mathrm{i} \alpha}\left(\begin{array}{cc}
1+\mathrm{i} \alpha & -1+\mathrm{i} \alpha \\
-1+\mathrm{i} \alpha & 1+\mathrm{i} \alpha
\end{array}\right) \in \mathrm{GL}_{2}(\mathbb{Q}(\mathrm{i} \alpha)) .
$$


It is easily checked that $M^{-1} \mathfrak{c}(M)$ is equal to $\rho(s)$ up to a scalar multiple (a general way to find such an $M$ will be described in section 8). Let $\rho^{\prime}: G \rightarrow \mathrm{GL}_{2}\left(K^{\prime}\right)$ be defined by $\rho^{\prime}(g)=M \rho(g) M^{-1}$. We have $\mathfrak{c}\left(\rho^{\prime}(g)\right)=M \rho(s) \mathfrak{c}(\rho(g)) \rho(s) M^{-1}=$ $M \rho(g) M^{-1}=\rho^{\prime}(g)$ hence $\rho^{\prime}(g) \in \mathrm{GL}_{2}(K)$. Moreover, for $\gamma \in H$ we have $\gamma(M)=$ $M$ hence $\gamma \circ \rho^{\prime}(g)=M \gamma \circ \rho(g) M^{-1}=M \circ \rho \circ \tilde{\iota}(\gamma)(g) M^{-1}=\rho^{\prime} \circ \tilde{\iota}(\gamma)(g)$. It follows that, in these cases, we have morphisms $\iota: \Gamma \hookrightarrow \operatorname{Aut}(G)$ and models over $K$ for all irreducible representations $\rho$ of $G$, such that $\gamma \circ \rho=\rho \circ \iota(\gamma)$ for all $\gamma \in \Gamma$. Thus, in those cases we can take $K^{\prime}=K$ in Theorems 1.6 and 1.7 .

7.2. The case $G(d, 1, r)$. The group $G=G(d, 1, r)$ is generated by the set of reflections $S=\left\{t, s_{1}, \ldots, s_{r-1}\right\}$ where $t=\operatorname{Diag}\left(\zeta_{d}, 1, \ldots, 1\right)$ and $s_{k}=M_{(k, k+1)}$ with the notations of the proof of Theorem 5.1 We have $\eta\left(\mathfrak{s}_{\alpha}\right)(t)=t^{\alpha}$ and $\eta\left(\mathfrak{s}_{\alpha}\right)\left(s_{k}\right)=$ $s_{k}$.

We provide an easy proof of Theorems 1.4 and 1.6 for $G(d, 1, r)$ starting from the case $r=2$. Then $G$ has order $2 d^{2}$, and its irreducible representations have dimension 1 and 2. The case of linear characters is immediate. The 2-dimensional representations are given, for $\zeta_{1}, \zeta_{2} \in \mu_{d}, \zeta_{1} \neq \zeta_{2}$, by the model

$$
\rho_{\zeta_{1}, \zeta_{2}}(t)=\left(\begin{array}{cc}
\zeta_{1} & 0 \\
0 & \zeta_{2}
\end{array}\right) \quad \rho_{\zeta_{1}, \zeta_{2}}\left(s_{1}\right)=\left(\begin{array}{cc}
0 & 1 \\
1 & 0
\end{array}\right) .
$$

It is readily seen that

$$
\rho_{\zeta_{1}, \zeta_{2}} \circ \eta\left(\mathfrak{s}_{\alpha}\right)=\mathfrak{s}_{\alpha} \circ \rho_{\zeta_{1}, \zeta_{2}}=\rho_{\zeta_{1}^{\alpha}, \zeta_{2}^{\alpha}}
$$

and this proves Theorems 1.4, 1.6 for the groups $G(d, 1,2)$ by taking $K^{\prime}=K$ and $\tilde{\iota}=\eta$. The first equality in the above formula also shows that the model is $\eta$-equivariant, thus giving 1.7

Theorems 1.4 and 1.6 are then easily deduced from this for the groups $G(d, 1, r)$, using that, for $r \geq 3$, each irreducible representation of $G(d, 1, r)$ is determined up to isomorphism by its restriction to the parabolic subgroup $G(d, 1, r-1)$ (see e.g. ArKo, Corollary 3.12).

We will give a proof of Theorem 1.7 for $G(d, 1, r)$ in the same spirit as in Proposition 9.2. But in the next subsections we will show more constructively that the models of 2.3 and 2.4 are $\tilde{\iota}_{\chi}$-equivariant for a suitable $\tilde{\iota}_{\chi}$.

7.3. The case of $G(d, 1, r)$. From the formulas $\eta\left(\mathfrak{s}_{\alpha}\right)(t)=t^{\alpha}$ and $\eta\left(\mathfrak{s}_{\alpha}\right)\left(s_{k}\right)=s_{k}$ and the formulae of subsection 2.3 , it is clear that our models over $\mathbb{Q}\left(\zeta_{d}\right)$ satisfy Theorem 1.6 by taking $\tilde{\iota}=\eta$; actually, the formulae show that the model $\rho_{\boldsymbol{\lambda}}$ is $\tilde{\imath}$-equivariant, which gives Theorem 1.7 .

More precisely, if we define the operator $\Sigma_{\alpha}: \mathcal{T}(\boldsymbol{\lambda}) \rightarrow \mathcal{T}\left(\mathfrak{s}_{\alpha}(\boldsymbol{\lambda})\right)$ by $\Sigma_{\alpha}(T)=$ $\left(T_{0}, T_{\alpha}, T_{2 \alpha}, \ldots, T_{(d-1) \alpha}\right)$, we have $\Sigma_{\alpha} \circ \mathfrak{s}_{\alpha} \circ \rho_{\boldsymbol{\lambda}}=\rho_{\mathfrak{s}_{\alpha}(\boldsymbol{\lambda})} \circ \Sigma_{\alpha}$ where $\rho_{\mathfrak{s}_{\alpha}(\boldsymbol{\lambda})}$ is as in Lemma 5.2

7.4. The general case $G(d e, e, r)$. Using that the matrix $S$ of subsection 2.4 is rational in our chosen basis of $V(\boldsymbol{\lambda})$, for $\omega \in \mu_{\# C_{\boldsymbol{\lambda}}}$ we get

$$
\mathfrak{s}_{\alpha}\left(\chi_{\boldsymbol{\lambda}, \omega}(g)\right)=\frac{1}{\# C_{\boldsymbol{\lambda}}} \sum_{i=0}^{\# C_{\boldsymbol{\lambda}}-1} \mathfrak{s}_{\alpha}(\omega)^{-i} \operatorname{Trace}\left(\mathfrak{s}_{\alpha}\left(\rho_{\boldsymbol{\lambda}}(g)\right) S^{i}\right) .
$$


From this, if we write $S_{\boldsymbol{\lambda}}$ for $S$ to keep track of where it acts, we get

$$
\mathfrak{s}_{\alpha}\left(\chi_{\boldsymbol{\lambda}, \omega}(g)\right)=\frac{1}{\# C_{\boldsymbol{\lambda}}} \sum_{i=0}^{\# C_{\boldsymbol{\lambda}}-1} \omega^{-i \alpha} \operatorname{Trace}\left(\Sigma_{\alpha}^{-1} \rho_{\mathfrak{s}_{\alpha}(\boldsymbol{\lambda})}(g) \Sigma_{\alpha} S_{\boldsymbol{\lambda}}^{i}\right) .
$$

Now, it is easy to check that $\Sigma_{\alpha} S_{\boldsymbol{\lambda}} \Sigma_{\alpha}^{-1}=S_{\mathfrak{s}_{\alpha}(\boldsymbol{\lambda})}^{\alpha}$, so

$$
\mathfrak{s}_{\alpha}\left(\chi_{\boldsymbol{\lambda}, \omega}(g)\right)=\frac{1}{\# C_{\boldsymbol{\lambda}}} \sum_{i=0}^{\# C_{\boldsymbol{\lambda}}-1} \omega^{-i \alpha} \operatorname{Trace}\left(\rho_{\mathfrak{s}_{\alpha}(\boldsymbol{\lambda})}(g) S_{\mathfrak{s}_{\alpha}(\boldsymbol{\lambda})}^{i \alpha}\right)=\chi_{\mathfrak{s}_{\alpha}(\boldsymbol{\lambda}), \omega}(g) ;
$$

while we have by a similar computation,

$$
\begin{aligned}
\chi_{\boldsymbol{\lambda}, \omega}\left(\tilde{l}\left(\mathfrak{s}_{\alpha}\right)(g)\right) & =\frac{1}{\# C_{\boldsymbol{\lambda}}} \sum_{i=0}^{\# C_{\boldsymbol{\lambda}}-1} \omega^{-i} \operatorname{Trace}\left(\mathfrak{s}_{\alpha}\left(\rho_{\boldsymbol{\lambda}}(g)\right) S_{\boldsymbol{\lambda}}^{i}\right) \\
& =\frac{1}{\# C_{\boldsymbol{\lambda}}} \sum_{i=0}^{\# C_{\boldsymbol{\lambda}}-1} \omega^{-i} \operatorname{Trace}\left(\rho_{\mathfrak{s}_{\alpha}(\boldsymbol{\lambda})}(g) S_{\mathfrak{s}_{\alpha}(\boldsymbol{\lambda})}^{i \alpha}\right) \\
& =\chi_{\mathfrak{s}_{\alpha}(\boldsymbol{\lambda}), \mathfrak{s}_{\alpha}-1(\omega)}(g) .
\end{aligned}
$$

We need slightly more precise formulas that we will get using our model which simplifies Ariki's model. We claim that if $\theta=\zeta_{e}^{b}$, then

$$
\mathfrak{s}_{\alpha} \circ \rho_{\boldsymbol{\lambda}, \theta^{i}}=\rho_{\boldsymbol{\lambda}, \theta^{i}} \circ \operatorname{Ad} t^{i(1-\alpha)} \circ \eta\left(\mathfrak{s}_{\alpha}\right)=\rho_{\boldsymbol{\lambda}, \theta^{i}} \circ \operatorname{Ad} t^{i} \circ \eta\left(\mathfrak{s}_{\alpha}\right) \circ \operatorname{Ad} t^{-i} ;
$$

indeed this formula is obvious for the generators $t$ and $s_{i}$ for $i \neq 1$. It is thus sufficient to check it for $s_{1}^{t^{k}}$; one uses the formulae (2.3) and the fact that $\rho_{\boldsymbol{\lambda}, 1}\left(s_{1}\right)$ is a rational matrix.

Let us define $\tilde{\iota}_{\chi_{\lambda, \theta i}}: \Gamma \rightarrow \operatorname{Aut}(G)$ by $\tilde{\iota}_{\chi_{\lambda, \theta i}}\left(\mathfrak{s}_{\alpha}\right)=\operatorname{Ad} t^{i} \circ \eta\left(\mathfrak{s}_{\alpha}\right) \circ \operatorname{Ad} t^{-i}$. The formula above becomes $\mathfrak{s}_{\alpha} \circ \rho_{\boldsymbol{\lambda}, \theta^{i}}=\rho_{\boldsymbol{\lambda}, \theta^{i}} \circ \tilde{\iota}_{\chi_{\boldsymbol{\lambda}, \theta^{i}}}\left(\mathfrak{s}_{\alpha}\right)$, i.e., that $\rho_{\boldsymbol{\lambda}, \theta^{i}}$ is $\tilde{\iota}_{\chi_{\boldsymbol{\lambda}, \theta^{i}}}$-equivariant, which proves Theorems 1.6 and 1.7. We note that the various morphisms $\tilde{\iota}_{\chi_{\lambda, \theta^{i}}}$ differ by conjugacy by an element of $N$. Indeed, they are conjugate by a power of Ad $t$ which is a generator of $N$. When $\operatorname{gcd}(e, r)=1$, then $\bar{N}$ is trivial, and when $\operatorname{gcd}(e, r)=2$, then $\bar{N}$ is of order 2 and the result is that $\tilde{\iota}_{\chi_{\boldsymbol{\lambda}, \theta i}}$ does not depend on $i$; indeed, $\alpha$ being prime to de is odd thus $1-\alpha$ is even and $\operatorname{Ad} t^{i(1-\alpha)}$ is always inner, which takes care of the remarks in Theorem 1.4

\section{Galois descent}

The existence of a model over $K$ globally invariant by $\Gamma=\operatorname{Gal}(K / \mathbb{Q})$ of a faithful representation of $G$, namely its reflection representation, enabled us in the $G(d e, e, r)$ case to construct the morphism $\tilde{\iota}: \Gamma \rightarrow \operatorname{Aut}(G)$.

In this section, we show, by Galois descent, how one can conversely try to construct a globally invariant model for any given irreducible representation - more precisely, we will try to get a $\tilde{\iota}$-equivariant model. The possible obstruction to do so is an element of the Brauer group of $K^{\prime}$ that turns out to always be trivial except for the group $G_{22}$.

8.1. Non-abelian Galois cohomology. Recall from [Se] that, if $H$ is a group acting on the left on a group $Q$, then a map $h \mapsto A_{h}$ from $H$ to $Q$ is a cocycle if $A_{s t}=A_{s} s\left(A_{t}\right)$ for all $s, t \in H$. A particular cocycle, called the zero cocycle, is given by sending all elements of $H$ to the trivial element of $Q$. The set of cocycles is denoted $Z^{1}(H, Q)$. Two cocycles $h \mapsto A_{h}$ and $h \mapsto B_{h}$ are said to be cohomologous 
if there exists $a \in Q$ such that $B_{h}=a^{-1} A_{h} h(a)$. This defines an equivalence relation on $Z^{1}(H, Q)$ whose set of equivalence classes is denoted $H^{1}(H, Q)$. The set of cocycles cohomologous to the zero cocycle is called the set of coboundaries and is denoted $B^{1}(H, Q)$. If $Q$ is commutative, these definitions coincide with those of ordinary group cohomology.

Application to Theorem 1.7. Let $\rho: G \rightarrow \mathrm{GL}(E)$ where $E$ is a $K^{\prime}$-vector space be an irreducible representation. Assume we choose a $\mathbb{Q}$-form $E=E_{0} \otimes_{\mathbb{Q}} K^{\prime}$. This defines an action of $\Gamma^{\prime}=\operatorname{Gal}\left(K^{\prime} / \mathbb{Q}\right)$ on $E$ and by Theorem 1.4 for $\gamma \in \Gamma^{\prime}$ we have $\gamma \circ \rho \simeq \rho \circ \tilde{\iota}(\gamma)$. This means that there exists $A_{\gamma} \in \mathrm{GL}(E)$ such that for any $g \in G$ we have $A_{\gamma} \gamma(\rho(g)) A_{\gamma}^{-1}=\rho(\tilde{\iota}(\gamma)(g))$. Since $\rho$ is absolutely irreducible, by Schur's lemma, $A_{\gamma}$ gives a well-defined element of $\operatorname{PGL}(E)$. It is immediate to check that $\gamma \mapsto A_{\gamma}$ is in fact a cocycle, that is, an element of $Z^{1}\left(\Gamma^{\prime}, \operatorname{PGL}(E)\right)$.

Assume now that $\rho$ has a globally invariant model of the form $g \mapsto a \rho(g) a^{-1}$; this means that there exists a map $\iota^{\prime}: \Gamma^{\prime} \rightarrow \operatorname{Aut}(G)$ such that $\gamma\left(a \rho(g) a^{-1}\right)=$ $a \rho\left(\iota^{\prime}(\gamma)(g)\right) a^{-1}$, or in other terms that the model is $\iota^{\prime}$-equivariant for some $\iota^{\prime}$ : $\Gamma^{\prime} \rightarrow \operatorname{Aut}(G)$. If $\iota^{\prime}=\tilde{\iota}$, we get the equality in $\operatorname{PGL}(E)$ that $a^{-1} \gamma(a)=A_{\gamma}$, that is, that $\left\{\gamma \mapsto A_{\gamma}\right\} \in B^{1}\left(\Gamma^{\prime}, \operatorname{PGL}(E)\right)$. Thus the obstruction to the existence of an i-equivariant model is an element of $H^{1}\left(\Gamma^{\prime}, \operatorname{PGL}(E)\right)$.

In practice, to apply this procedure we encounter another problem which turns out to involve also Galois cohomology; although we know that any $\rho$ has a model over $K^{\prime}$ (even $K$ ), sometimes (e.g. when using the CHEVIE data) we are not given such a model, but a model $E=E_{0} \otimes_{K^{\prime}} L$ over some Galois extension $L$ of $K^{\prime}$. Again the fact that for $\gamma \in \Pi=\operatorname{Gal}\left(L / K^{\prime}\right)$ we have $\gamma \circ \rho \simeq \rho$ means that we can find an intertwiner $A_{\gamma}$ such that $A_{\gamma} \gamma(\rho(g)) A_{\gamma}^{-1}=\rho(g)$, which is an element of $Z^{1}(\Pi, \mathrm{PGL}(E))$; and again the existence of a model over $K^{\prime}$ is equivalent to this cocycle to be a coboundary.

We will now give an algorithm to check the vanishing of a cocycle of a Galois group into the projective linear group.

8.2. Brauer groups. In this subsection and the next one, $K_{0} \subseteq K$ will denote an arbitrary Galois extension of number fields. Let $\Gamma=\operatorname{Gal}\left(K / K_{0}\right)$ and let $\operatorname{Br}\left(K / K_{0}\right)=H^{2}\left(\Gamma, K^{\times}\right)$be the Brauer group. Let $E=E_{0} \otimes_{K_{0}} K$ be a $K_{0}$-form of a finite dimensional $K$-vector space $E$. The short exact sequence $1 \rightarrow K^{\times} \rightarrow \mathrm{GL}(E) \rightarrow \mathrm{PGL}(E) \rightarrow 1$ gives rise to a Galois cohomology long exact sequence (of pointed sets; see [Se, Prop. 2, p. 133]):

$$
\begin{aligned}
1 \rightarrow H^{0}\left(\Gamma, K^{\times}\right) \rightarrow H^{0}(\Gamma, \mathrm{GL}(E)) \rightarrow H^{0}(\Gamma, \operatorname{PGL}(E)) \rightarrow H^{1}\left(\Gamma, K^{\times}\right) & \\
& \rightarrow H^{1}(\Gamma, \mathrm{GL}(E)) \rightarrow H^{1}(\Gamma, \operatorname{PGL}(E)) \rightarrow H^{2}\left(\Gamma, K^{\times}\right)
\end{aligned}
$$

and, in particular, a coboundary operator $H^{1}(\Gamma, \operatorname{PGL}(E)) \rightarrow H^{2}\left(\Gamma, K^{\times}\right)$. This map sends $\left\{\gamma \mapsto A_{\gamma}\right\} \in H^{1}(\Gamma, \operatorname{PGL}(E))$ to the class of $(\gamma, \tau) \mapsto \tilde{A}_{\gamma} \gamma\left(\tilde{A}_{\tau}\right) \tilde{A}_{\gamma \tau}^{-1}$ in $H^{2}\left(\Gamma, K^{\times}\right)$, where, for all $\gamma \in \Gamma, \tilde{A}_{\gamma}$ is a representative of $A_{\gamma}$ in $\operatorname{GL}(E)$. This definition does not depend on the choice of the representatives $\tilde{A}_{\gamma}$ and this map is known to be injective (this uses the version of Hilbert's Theorem 90 which says that $H^{1}(\Gamma, \mathrm{GL}(E))$ is trivial; see [Se ch. X, Proposition 8 and Proposition 9).

In the important case where $K$ is a cyclic extension of degree $n$ of $K_{0}$, assume $\Gamma=$ $\langle\gamma\rangle$ and let $N: K \rightarrow K_{0}$ be the norm map $x \mapsto x \gamma(x) \ldots \gamma^{n-1}(x)$. Then an explicit 
isomorphism from $H^{2}\left(\Gamma, K^{\times}\right)$to the 0 -th Tate cohomology group $\hat{H}^{0}\left(\Gamma, K^{\times}\right)=$ $K_{0}^{\times} / N K^{\times}$is given by the Nakayama map

$$
c \mapsto \prod_{k=0}^{n-1} c\left(\gamma^{k}, \gamma\right),
$$

hence $\left\{\gamma \mapsto A_{\gamma}\right\}$ is sent to the class of $\tilde{A}_{\gamma} \gamma\left(\tilde{A}_{\gamma}\right) \ldots \gamma^{n-1}\left(\tilde{A}_{\gamma}\right)$ (a scalar matrix identified to an element of $\left.K_{0}^{\times}\right)$in $K_{0}^{\times} / N K^{\times}$.

It follows that, when $K$ is a cyclic extension of $K_{0}$, the vanishing of $c \in H^{2}\left(\Gamma, K^{\times}\right)$ $=\operatorname{Br}\left(K / K_{0}\right)$ boils down to the verification that some element of $K_{0}^{\times}$is a norm of an element of $K$. When $K$ is an abelian extension of $K_{0}$, we can always reduce to this case by induction on $\left[K: K_{0}\right]$, by the inflation-restriction exact sequence for Brauer groups. Indeed, choosing a cyclic subgroup $\Gamma^{\prime}$ of $\Gamma$ defines a Galois sub-extension $K_{1}=K^{\Gamma^{\prime}}$ of $K$ such that $\operatorname{Gal}\left(K / K_{1}\right)$ is cyclic. We then have the exact sequence (see [Se] ch. X, $\S 4$, Proposition 6)

$$
0 \rightarrow \operatorname{Br}\left(K_{1} / K_{0}\right) \rightarrow \operatorname{Br}\left(K / K_{0}\right) \rightarrow \operatorname{Br}\left(K / K_{1}\right)
$$

and we may follow the following procedure: first check that the image in $\operatorname{Br}\left(K / K_{1}\right)$ is zero, and then start again with the induced element in $\operatorname{Br}\left(K_{1} / K_{0}\right)$, until $K_{1} / K_{0}$ itself becomes a cyclic extension.

We finally mention the following general result.

Proposition 8.1. If \# $\Pi$ is prime to $\operatorname{dim}(E)$, then $H^{1}(\Gamma, \operatorname{PGL}(E))=0$.

Proof. Let $N=\operatorname{dim}(E)$. Let $c \in H^{1}(\Gamma, \mathrm{PGL}(E))$ and let $A$ be the central simple algebra associated to it. Let $e$ be its exponent, that is, the order of its class $[A]$ in the Brauer group of $K_{0}$. Since $A \otimes K \simeq M_{N}(K)$ we have $[A][K]=0$ in the Brauer group hence $e \mid\left[K: K_{0}\right]$ and $e \mid \# \Gamma$ (see $[\mathrm{Se}, \mathrm{X}, \S 4$, ex. 2). On the other hand, $A \simeq M_{r}(D)$ with $D$ a skew field whose center is $K_{0}$ and $\operatorname{dim}_{K_{0}} A=N^{2}$. It follows that $N^{2}=\left[A: K_{0}\right]=r^{2}\left[D: K_{0}\right]$. Now $\left[D: K_{0}\right]=m^{2}$ for some integer $m$ (called the index of $D$ ). It is a classical fact that the exponent divides the index ( $\mathrm{Se}$, $\mathrm{X}, \S 5$, ex. 3a), thus $e \mid N$, and $e=1$ since $N$ and $\# \Gamma$ are coprime. The conclusion follows from the fact that $H^{1}(\Gamma, \mathrm{PGL}(E))$ embeds in the Brauer group of $K_{0}$.

Explicit computations. In practice (e.g., to get an explicit model over a smaller field of a representation), it is not enough to solve the problem of whether a cocycle $\left\{\gamma \mapsto A_{\gamma}\right\} \in Z^{1}(\Gamma, \mathrm{PGL}(E))$ is a coboundary. We want an explicit element $M \in$ $\operatorname{PGL}(E)$ such that $A_{\gamma}=M^{-1} \gamma(M)$ for all $\gamma \in \Gamma$. If we can get a preimage $\left(B_{\gamma}\right)$ of $\left(A_{\gamma}\right)$ in $Z^{1}(\Gamma, \mathrm{GL}(E))$, then by Hilbert's Theorem 90 this cocycle is a coboundary, meaning that there exist $\tilde{M} \in \mathrm{GL}(E)$ such that $B_{\gamma}=\tilde{M}^{-1} \gamma(\tilde{M})$ for all $\gamma \in \Gamma$. A rather constructive proof of this theorem (see e.g. Se, ch. X, Proposition 3) goes as follows. For all $C \in \operatorname{End}(E)$, the expression

$$
X=\sum_{\gamma \in \Gamma} B_{\gamma} \gamma(C)
$$

satisfies $\gamma(X)=B_{\gamma}^{-1} X$. Because $K$ has characteristic zero, general arguments imply that "many" $C \in K$ exist such that $X$ is invertible. If this is the case, then $B_{\gamma}=X \gamma(X)^{-1}$ thus $\tilde{M}=X^{-1}$ is the desired solution. For instance, if $\operatorname{Gal}\left(K / K_{0}\right)=\{1, \gamma\}$ has order 2 with generator $\gamma_{0}$, then the condition on $C$ is that $-C \gamma(C)$ is not an eigenvalue for $B_{\mathrm{Id}}^{-1} B_{\gamma_{0}}$. 
In the case where $K$ is a cyclic extension of $K_{0}$, the study of the previous section shows that we can always lift a coboundary to a cocycle $\left\{\gamma \mapsto B_{\gamma}\right\}$ for $\operatorname{GL}(E)$. Indeed, if $\Gamma=\langle\gamma\rangle$ we have seen that $\tilde{A}_{\gamma} \gamma\left(\tilde{A}_{\gamma}\right) \ldots \gamma^{n-1}\left(\tilde{A}_{\gamma}\right)=N(\lambda)$ Id for some $\lambda \in K^{\times}$so that $B_{\gamma}=\lambda^{-1} \tilde{A}_{\gamma}$ belongs to $Z^{1}(\Gamma, \mathrm{GL}(E))$ (it is easy to check that the equation Id $=\tilde{A}_{\gamma} \ldots \gamma^{n-1}\left(\tilde{A}_{\gamma}\right)$ is necessary and sufficient for $\tilde{A}_{\gamma^{i}}=\tilde{A}_{\gamma} \ldots \gamma^{i-1}\left(\tilde{A}_{\gamma}\right)$ to define a cocycle of $\langle\gamma\rangle)$.

8.3. An algorithm to check Theorem 1.7. Let $\rho: G \rightarrow \mathrm{GL}(E)$ be an irreducible representation of $G$ over the $K^{\prime}$-vector space $E$ for which we would like to get a $\tilde{\imath}$-equivariant model. When $K^{\prime} / \mathbb{Q}$ is cyclic, we found an explicit necessary and sufficient condition for a class in $H^{1}\left(\Gamma^{\prime}, \operatorname{PGL}(E)\right)$ to vanish and thus to give an equivariant model.

In general we know that $K^{\prime} / \mathbb{Q}$ is an abelian extension. We will show how to reduce by induction to the case of a cyclic extension, by making explicit the inflationrestriction exact sequence in this case.

Choose a tower of (Galois) extensions $\mathbb{Q}=K_{0} \subset K_{1} \subset \cdots \subset K_{m}=K^{\prime}$ such that $K_{r} / K_{r-1}$ is a cyclic extension for all $1 \leq r \leq m$. We fix a $\mathbb{Q}$-form $E_{0}$ of $E$ and a basis of $E_{0}$ so that we identify $E_{0}$ with $\mathbb{Q}^{n}$ and $E$ with $K^{\prime n}$.

Assume that, for some $r \in[1, m]$ we have a model $\rho_{r}: G \rightarrow \operatorname{GL}(E)$ of $\rho$ such that $\gamma \circ \rho=\rho \circ \iota(\gamma)$ for all $\gamma \in \operatorname{Gal}\left(K^{\prime} / K_{r}\right)$. We will show how to decrease $r$.

Let $\left\{\tau \mapsto M_{\tau}\right\} \in Z^{1}\left(\operatorname{Gal}\left(K^{\prime} / K_{r-1}\right), \operatorname{PGL}(E)\right)$ intertwining $\tau \circ \rho_{r}$ and $\rho_{r} \circ \iota(\tau)$ for $\tau \in \operatorname{Gal}\left(K^{\prime} / K_{r-1}\right)$. For $\gamma$ in the subgroup $\operatorname{Gal}\left(K^{\prime} / K_{r}\right)$ we have $M_{\gamma}=1$ by the induction hypothesis; we thus have $M_{\tau \sigma}=M_{\tau}$ for all $\sigma \in \operatorname{Gal}\left(K^{\prime} / K_{r}\right)$ by the cocycle condition $M_{\tau \gamma}=M_{\tau} \tau\left(M_{\gamma}\right)$. Hence $M_{\tau}$ only depends on the class of $\tau$ in $\operatorname{Gal}\left(K_{r} / K_{r-1}\right)$, and lies in $\mathrm{PGL}_{n}\left(K_{r}\right)$ because $M_{\tau}=M_{\tau \gamma}=M_{\gamma \tau}=M_{\gamma} \gamma\left(M_{\tau}\right)=$ $\gamma\left(M_{\tau}\right)$ for all $\gamma \in \operatorname{Gal}\left(K^{\prime} / K_{r}\right)$. It follow that $\left\{\tau \mapsto M_{\tau}\right\}$ defines an element of $Z^{1}\left(\operatorname{Gal}\left(K_{r} / K_{r-1}\right), \mathrm{PGL}_{n}\left(K_{r}\right)\right)$; we are thus reduced to a cyclic case; assuming we can solve it we find $X \in \mathrm{GL}_{n}\left(K_{r}\right)$ such that $M_{\tau}=X \tau(X)^{-1}$ for all $\tau \in$ $\operatorname{Gal}\left(K^{\prime} / K_{r-1}\right)$. Then $\rho_{r-1}(g)=X \rho_{r}(g) X^{-1}$ is a model of $\rho$ such that $\gamma \circ \rho_{r-1}=$ $\rho_{r-1} \circ \iota(\gamma)$ for all $\gamma \in \operatorname{Gal}\left(K^{\prime} / K_{r-1}\right)$.

We could successfully carry out this algorithm for all representations of exceptional groups for which we had a model. However, in contrast to the cyclic case, we do not get in general necessary and sufficient conditions for a class in $H^{1}\left(\Gamma^{\prime}, \mathrm{PGL}(E)\right)$ to vanish. Note, however, the following observation:

Proposition 8.2. Assume $G$ to be an exceptional irreducible complex reflection group. Then any odd-dimensional irreducible representation has a globally invariant model over $K^{\prime}$.

Proof. A case-by-case analysis shows that $\left[K^{\prime}: \mathbb{Q}\right]$ is always a power of 2 . The result is then an immediate consequence of Proposition 8.1.

\section{INVARIANT MODELS}

We will now prove Theorem 1.7 using the results of the previous sections and multiplicity one property of tensor products.

For two representations $\rho_{1}, \rho_{2}$ of a group $G$, we will denote $\left(\rho_{1} \mid \rho_{2}\right)$ the dimension of the space of intertwiners of $\rho_{1}$ and $\rho_{2}$. This coincides with the usual scalar product of the corresponding characters.

We will make repeated use of the following lemma. 
Lemma 9.1. Let $G$ be a finite group and $K$ be a field of characteristic 0 . Let $\rho^{\prime}$ be a finite-dimensional representation of $G$ defined over $K$ and let $\rho \in \operatorname{Irr}(G)$ be such that $\left(\rho^{\prime} \mid \rho\right)=1$. Let $\chi$ be the character of $\rho$. Assume that $\chi$ takes values in $K$. Then $\rho$ admits a model over $K$ and:

- if $\gamma \in \operatorname{Gal}(K / \mathbb{Q})$ and $a \in \operatorname{Aut}(G)$ satisfy $\gamma \circ \rho^{\prime}=\rho^{\prime} \circ a$ and $\gamma \circ \chi=\chi \circ a$, then $\rho$ admits a model over $K$ such that $\gamma \circ \rho=\rho \circ$ a;

- if there exists $j: \operatorname{Gal}(K / \mathbb{Q}) \rightarrow \operatorname{Aut}(G)$ such that $\gamma \circ \rho^{\prime}=\rho^{\prime} \circ j(\gamma)$ and $\gamma \circ \chi=\chi \circ j(\gamma)$, then $\rho$ admits a $j$-equivariant model over $K$.

Proof. Since $\rho$ is an isotypic component of a $\rho^{\prime}$ and has its character over $K$ it is defined over $K$; indeed, the $G$-equivariant projector

$$
p=\frac{\chi(1)}{\# G} \sum_{g \in G} \chi\left(g^{-1}\right) \rho^{\prime}(g)
$$

on the $\rho$-isotypic component of $\rho^{\prime}$ belongs to $\operatorname{End}_{K}(V)$. Moreover, if $\gamma \circ \chi=\chi \circ a$, we have

$$
\gamma(p)=\frac{\chi(1)}{\# G} \sum_{g \in G} \chi\left(a(g)^{-1}\right) \rho^{\prime}(a(g))=p,
$$

thus a $\mathbb{Q}$-form $V^{\prime}=V_{0}^{\prime} \otimes_{\mathbb{Q}} K$ induces a $\mathbb{Q}$-form on $V=\operatorname{Im} p$. It follows that $\gamma \circ \rho=\rho \circ a$, which proves the last two points.

9.1. Reflection representations. We now prove Theorem 1.7 for the reflection representation of exceptional groups, that is the following proposition.

Proposition 9.2. Let $G$ be an exceptional irreducible complex reflection different from $G_{22}$ given by $\rho: G \hookrightarrow \mathrm{GL}(V)$ where $V$ is a $K$-vector space and $K$ is the field of the definition of $G$. Let $K^{\prime}$ be as in Theorem 1.6. Then $\rho$ has a $\tilde{\iota}_{\rho}$-equivariant model over $K^{\prime}$.

Recall that $K=K^{\prime}$ except when $G=G_{27}$.

Models for reflection representations of 2-dimensional groups from the braid group. A way to obtain the 2-dimensional representations of the groups $G_{4}$ to $G_{22}$ is by using the following matrices:

$$
\mathbf{s} \mapsto\left(\begin{array}{cc}
x_{1} & \frac{y_{1}+y_{2}}{y_{1} y_{2}}-\frac{\left(z_{1}+z_{2}\right) x_{2}}{r} \\
0 & x_{2}
\end{array}\right), \mathbf{t} \mapsto\left(\begin{array}{cc}
y_{1}+y_{2} & 1 / x_{1} \\
-y_{1} y_{2} x_{1} & 0
\end{array}\right), \mathbf{u} \mapsto\left(\begin{array}{cc}
0 & \frac{-r}{y_{1} y_{2} x_{1} x_{2}} \\
r & z_{1}+z_{2}
\end{array}\right)
$$

where $r=\sqrt{x_{1} x_{2} y_{1} y_{2} z_{1} z_{2}}$. The braid groups of $G_{7}, G_{11}$ and $G_{19}$ are isomorphic to the same group $B$, with presentation $\langle\mathbf{s}, \mathbf{t}, \mathbf{u} \mid \mathbf{s t u}=\mathbf{t u s}=\mathbf{u s t}\rangle$; the above matrices give the 2-dimensional representation of $B$ where the eigenvalues of $\mathbf{s}$ (resp. $\mathbf{t}$, u) are $x_{1}, x_{2}$ (resp. $y_{1}, y_{2}, z_{1}, z_{2}$ ). This representation factors through the Hecke algebra, the quotient of the group algebra of $B$ by the relations $\left(\mathbf{s}-x_{0}\right)\left(\mathbf{s}-x_{1}\right)=0$, $\left(\mathbf{t}-y_{0}\right)\left(\mathbf{t}-y_{1}\right)\left(\mathbf{t}-y_{2}\right)=0$ and $\prod_{i=0}^{i=n-1}\left(\mathbf{u}-z_{i}\right)=0$ where $n=3$ (resp. 4,5) for $G_{7}$ (resp. $G_{11}, G_{19}$ ). In turn the group algebra of $G_{7}$ (resp. $G_{11}, G_{19}$ ) is the specialization of the Hecke algebra for $x_{i} \mapsto(-1)^{i}, y_{i} \mapsto \zeta_{3}^{i}, z_{i} \mapsto \zeta_{3}^{i}$ (resp. $z_{i} \mapsto \zeta_{4}^{i}$, $\left.z_{i} \mapsto \zeta_{5}^{i}\right)$; the Hecke algebras for $G_{4}$ to $G_{6}$ are subalgebras of partial specializations of that for $G_{7}$ (the same holds for $G_{8}$ to $G_{15}$ with respect to $G_{11}$ and $G_{16}$ to $G_{22}$ with respect to $G_{19}$ ): in each case, these algebras are generated by conjugates of part of the generators (or of some power of them); the other generators are specialized to the group algebra. 
For $G_{4}, G_{8}$ and $G_{16}$ the Hecke algebra is generated by $\mathbf{u}$ and ${ }^{\mathbf{s}} \mathbf{u}$.

For $G_{5}, G_{10}$ and $G_{18}$ it is generated by $\mathbf{t}$ and $\mathbf{u}$.

For $G_{6}, G_{9}$ and $G_{17}$ it is generated by $\mathbf{s}$ and $\mathbf{u}$.

For $G_{14}$ and $G_{21}$ it is generated by $\mathbf{s}$ and $\mathbf{t}$.

For $G_{12}$ and $G_{22}$ it is generated by $\mathbf{s},{ }^{\mathbf{t}} \mathbf{s}$ and $\mathbf{s}^{\mathbf{t}}$.

For $G_{20}$ it is generated by $\mathbf{t}$ and $\mathbf{s}_{\mathbf{t}}$.

For $G_{13}$ it is generated by $\mathbf{u}^{2}, \mathbf{s}$ and $\mathbf{s}^{\mathbf{t}}$.

Finally, for $G_{15}$ it is generated by $\mathbf{s}, \mathbf{t}$ and $\mathbf{u}^{2}$.

Proof of Proposition 9.2. The statement is clear if the rank of $G$ is odd, by Proposition 8.2. Then we may assume that, either $G=G_{34}$, or that its rank is 2 or 4 . Recall that the groups of rank 2 are numbered $G_{4}, \ldots, G_{22}$.

We started from a model of the reflection representations coming either from the Hecke algebra as described above, or by root diagrams as considered in Cohen. Then we apply the algorithm of Galois descent; in order to solve norm equations we used the computer system MAGMA. All equations thus obtained for the reflection representation were solvable except for $G_{22}$.

Note that, even when the original model is globally invariant, the induced morphism $\eta: \Gamma \rightarrow \operatorname{Aut}(G)$ does not in general coincide with $\tilde{\iota}_{\rho}$. Furthermore, the induced action of $\eta$ on the isomorphism classes of irreducible representations of $G$ may also differ from the natural Galois action of $\Gamma$, as shown in the second example below.

The models given in Table 2 were simplified by the following elementary observation. For all the images $\tilde{\iota}(\Gamma)$ chosen here, it turns out that there is a generator $s$ such that the subgroup $H$ generated by $s$ is stable under $\tilde{\iota}(\Gamma)$. Lemma 9.1 thus implies that there is a basis of the underlying $\mathbb{Q}$-form of $K^{2}$ on which the action of $s$ is diagonal.

Table 2: Invariant models for $G_{4}$ to $G_{21}$

\begin{tabular}{|l|l|}
\hline$G_{4}$ & $s \mapsto\left(\begin{array}{cc}1 & 0 \\
0 & \zeta_{3}\end{array}\right), \quad t \mapsto \frac{1}{\sqrt{-3}}\left(\begin{array}{cc}-1 & \zeta_{3} \\
2 & \zeta_{3}\end{array}\right)$ \\
\hline$G_{5}$ & $s \mapsto\left(\begin{array}{cc}1 & 0 \\
0 & \zeta_{3}\end{array}\right), \quad t \mapsto \frac{1}{\sqrt{-3}}\left(\begin{array}{cc}\zeta_{3} & \zeta_{3} \\
2 & -1\end{array}\right)$ \\
\hline$G_{6}$ & $s \mapsto \frac{1}{\sqrt{3}}\left(\begin{array}{cc}1 & 1 \\
2 & -1\end{array}\right), \quad t \mapsto\left(\begin{array}{cc}1 & 0 \\
0 & \zeta_{3}\end{array}\right)$ \\
\hline$G_{7}$ & $s \mapsto \frac{1}{\sqrt{3}}\left(\begin{array}{cc}1 & 1 \\
2 & -1\end{array}\right), \quad t \mapsto\left(\begin{array}{cc}1 & 0 \\
0 & \zeta_{3}\end{array}\right), \quad u \mapsto \frac{1}{\sqrt{-3}}\left(\begin{array}{cc}\zeta_{3} & \zeta_{3} \\
2 & -1\end{array}\right)$ \\
\hline$G_{8}$ & $s \mapsto\left(\begin{array}{cc}1 & 0 \\
0 & \mathrm{i}\end{array}\right), \quad t \mapsto \frac{\mathrm{i}-1}{2}\left(\begin{array}{cc}-\mathrm{i} & 1 \\
1 & -\mathrm{i}\end{array}\right)$ \\
\hline$G_{9}$ & $s \mapsto \frac{1}{2}\left(\begin{array}{cc}\sqrt{2} & 2 \\
1 & -\sqrt{2}\end{array}\right), \quad t \mapsto\left(\begin{array}{cc}1 & 0 \\
0 & \mathrm{i}\end{array}\right)$ \\
\hline
\end{tabular}




\begin{tabular}{|c|c|}
\hline$G_{10}$ & $s \mapsto \frac{1}{\zeta_{3}(\mathrm{i}-1)}\left(\begin{array}{cc}-\mathrm{i} & 2 \mathrm{i} \\
\frac{1}{2} & 1\end{array}\right), \quad t \mapsto\left(\begin{array}{cc}1 & 0 \\
0 & \mathrm{i}\end{array}\right)$ \\
\hline$G_{11}$ & $s \mapsto C=\frac{1}{\sqrt{6}}\left(\begin{array}{cc}-2 & 1 \\
2 & 2\end{array}\right), \quad t \mapsto\left(\begin{array}{cc}1 & 0 \\
0 & \zeta_{3}\end{array}\right), \quad u \mapsto \frac{\zeta_{3}}{(\mathrm{i}+1) \sqrt{3}}\left(\begin{array}{cc}-2 \zeta_{3} & \zeta_{3} \\
2 & 2\end{array}\right)$ \\
\hline$G_{12}$ & $s \mapsto D=\frac{1}{2}\left(\begin{array}{cc}1 & 1+\frac{1}{\sqrt{-2}} \\
2+\sqrt{-2} & -1\end{array}\right), \quad t \mapsto \bar{D}, \quad u \mapsto\left(\begin{array}{cc}1 & 0 \\
0 & -1\end{array}\right)$ \\
\hline$G_{13}$ & $s \mapsto\left(\begin{array}{cc}1 & 0 \\
0 & -1\end{array}\right), \quad t \mapsto \frac{1}{\sqrt{2}}\left(\begin{array}{cc}1 & -1 \\
-1 & -1\end{array}\right), \quad u \mapsto \frac{1}{\sqrt{2}}\left(\begin{array}{cc}1 & -\mathrm{i} \\
\mathrm{i} & -1\end{array}\right)$ \\
\hline$G_{14}$ & $s \mapsto\left(\begin{array}{cc}1 & 0 \\
0 & -1\end{array}\right), \quad t \mapsto \frac{\zeta_{3}^{2}}{2}\left(\begin{array}{cc}-1+\sqrt{-2} & 1 \\
-1 & -1-\sqrt{-2}\end{array}\right)$ \\
\hline$G_{15}$ & $s \mapsto C, \quad t \mapsto\left(\begin{array}{cc}1 & 0 \\
0 & \zeta_{3}\end{array}\right), \quad u \mapsto \frac{1}{\sqrt{3}}\left(\begin{array}{cc}1 & \zeta_{3}^{2} \\
2 \zeta_{3} & -1\end{array}\right)$ \\
\hline$G_{16}$ & $s \mapsto\left(\begin{array}{cc}1 & 0 \\
0 & \zeta_{5}\end{array}\right), \quad t \mapsto \frac{1}{\sqrt{5}}\left(\begin{array}{cc}1-\zeta_{5}^{3} & \zeta_{5}^{4}-1 \\
\zeta_{5}-\zeta_{5}^{2} & \zeta_{5}-\zeta_{5}^{3}\end{array}\right)$ \\
\hline$G_{17}$ & $s \mapsto M=\frac{\mathrm{i}}{\sqrt{5}}\left(\begin{array}{cc}\zeta_{5}^{4}-\zeta_{5} & \zeta_{5}^{3}-\zeta_{5}^{2} \\
\zeta_{5}^{3}-\zeta_{5}^{2} & \zeta_{5}-\zeta_{5}^{4}\end{array}\right), \quad t \mapsto\left(\begin{array}{cc}1 & 0 \\
0 & \zeta_{5}\end{array}\right)$ \\
\hline$G_{18}$ & $s \mapsto N=\frac{\zeta_{3}^{2}}{\sqrt{5}}\left(\begin{array}{cc}\zeta_{5}^{2}-\zeta_{5}^{4} & 1-\zeta_{5}^{4} \\
\zeta_{5}-1 & \zeta_{5}^{3}-\zeta_{5}\end{array}\right), \quad t \mapsto\left(\begin{array}{cc}1 & 0 \\
0 & \zeta_{5}\end{array}\right)$ \\
\hline$G_{19}$ & $s \mapsto N, \quad t \mapsto M, \quad u \mapsto\left(\begin{array}{cc}1 & 0 \\
0 & \zeta_{5}\end{array}\right)$ \\
\hline$G_{20}$ & $s \mapsto\left(\begin{array}{cc}1 & 0 \\
0 & \zeta_{3}\end{array}\right), \quad t \mapsto \frac{\zeta_{3}^{2}}{2 \sqrt{-15}}\left(\begin{array}{cc}-5-\sqrt{-15} & 2 \\
10 & 5-\sqrt{-15}\end{array}\right)$ \\
\hline$G_{21}$ & $s \mapsto \frac{1}{2 \sqrt{3}}\left(\begin{array}{cc}1+\sqrt{5} & -1+\frac{1}{\sqrt{5}} \\
-5+\sqrt{5} & -1-\sqrt{5}\end{array}\right), \quad t \mapsto\left(\begin{array}{cc}1 & 0 \\
0 & \zeta_{3}\end{array}\right)$ \\
\hline
\end{tabular}

Example: the case of $G_{9}$. Let us start with the model

$$
s \mapsto\left(\begin{array}{cc}
0 & -\zeta_{24}^{11} \\
\zeta_{24} & 0
\end{array}\right), \quad t \mapsto\left(\begin{array}{cc}
\mathrm{i} & -\zeta_{3} \\
0 & 1
\end{array}\right)
$$

which is defined over $\mathbb{Q}\left(\zeta_{24}\right)$, an extension of degree 2 of $K=\mathbb{Q}\left(\zeta_{8}\right)$. By Galois descent or directly one finds that conjugating by $\left(\begin{array}{ll}1 & 0 \\ 0 & \zeta_{3}\end{array}\right)$ yields the following model over $K$.

$$
s \mapsto\left(\begin{array}{cc}
0 & -\zeta_{8} \\
\zeta_{8}^{3} & 0
\end{array}\right), \quad t \mapsto\left(\begin{array}{cc}
\mathrm{i} & -1 \\
0 & 1
\end{array}\right)
$$

We have $\Gamma \simeq \mathbb{Z} / 2 \times \mathbb{Z} / 2$, with three subgroups of order 2 generated by $\mathfrak{s}_{-1}, \mathfrak{s}_{5}$ and $\mathfrak{s}_{3}$. To these three subgroups correspond three intermediate extensions between $\mathbb{Q}$ 
and $K$. For each of these extensions, we get a cocycle $\mathfrak{s}_{k} \mapsto A_{k} \in G L_{2}(K)$, given by the following matrices:

$$
A_{-1}=\left(\begin{array}{cc}
-\mathrm{i} & 0 \\
0 & 1
\end{array}\right), \quad A_{5}=\left(\begin{array}{cc}
-1 & -1-\mathrm{i} \\
0 & 1
\end{array}\right), \quad A_{3}=\left(\begin{array}{cc}
\mathrm{i} & -1+\mathrm{i} \\
0 & 1
\end{array}\right) .
$$

It turns out that the last step of the algorithm is easy, because these three cocycles match, so we get in fact an element of $Z^{1}\left(\Gamma, \mathrm{GL}_{2}(K)\right)$. In order to apply Hilbert Theorem 90 we need to find $\lambda \in K$ such that $\lambda+\mathfrak{s}_{-1}(\lambda) A_{1}+\mathfrak{s}_{5}(\lambda) A_{2}+\mathfrak{s}_{3}(\lambda) A_{3}$ is invertible; it happens that $\lambda=1+\zeta_{8}+\zeta_{8}^{3}$ is a solution that gives the following invariant model:

$$
s \mapsto \frac{1}{2}\left(\begin{array}{cc}
-1-\zeta_{8}+\zeta_{8}^{3} & 1 / 2-\zeta_{8}+\zeta_{8}^{3} \\
2 & 1+\zeta_{8}-\zeta_{8}^{3}
\end{array}\right), \quad t \mapsto\left(\begin{array}{cc}
\mathrm{i} & \frac{\mathrm{i}-1}{2} \\
0 & 1
\end{array}\right) .
$$

Finally, we know that the subgroup generated by $t$ is stable by $\tilde{\imath}(\Gamma)$. It is thus possible to diagonalize it by a rational matrix. We get the still invariant and simpler model

$$
s \mapsto \frac{1}{2}\left(\begin{array}{cc}
\sqrt{2} & 2 \\
1 & -\sqrt{2}
\end{array}\right), \quad t \mapsto\left(\begin{array}{ll}
1 & 0 \\
0 & \mathrm{i}
\end{array}\right) .
$$

An example for $G_{7}$. The model $\rho$ of the reflection representation of $G_{7}$ given by

$$
s \mapsto\left(\begin{array}{cc}
1 & 0 \\
0 & -1
\end{array}\right), \quad t \mapsto \frac{\zeta_{3}^{2}}{(1+\mathrm{i})}\left(\begin{array}{cc}
-1 & -1 \\
\mathrm{i} & -\mathrm{i}
\end{array}\right), \quad u \mapsto \frac{\zeta_{3}^{2}}{(1+\mathrm{i})}\left(\begin{array}{cc}
-1 & \mathrm{i} \\
-1 & -\mathrm{i}
\end{array}\right)
$$

is globally invariant, giving rise to a homomorphism $\eta: \operatorname{Gal}\left(\mathbb{Q}\left(\zeta_{12}\right) / \mathbb{Q}\right) \rightarrow \operatorname{Aut}\left(G_{7}\right)$ given by (specifying automorphisms, as in Table 1 by the images of $s, t, u) \mathfrak{c} \mapsto$ $\left(s, u^{-1}, t^{-1}\right), \mathfrak{s}_{7} \mapsto\left(s, s^{u s}, u^{t s}\right)$. However, the automorphism $\eta(\mathfrak{c})$ is not compatible with the action of the complex conjugation on other characters of $G$, contrary to the homomorphism $\tilde{\iota}$ given in Table 1, which corresponds to the model given in Table 2 The existence of this example is related to the fact that $G_{7}$ is the only exceptional group such that the image $\bar{\iota}(\Gamma)$ does not commute with $N$.

9.2. Proof of Theorem 1.7 for exceptional groups. In this section we will deduce the existence of an $\tilde{\iota}$-equivariant model for every irreducible representation of $G$ (outside the exceptional set of Theorem 1.4) from the existence of such a model for the reflection representation $\rho_{0}$, by applying Lemma 9.1. The arguments that we shall present also provide a new proof of Theorem 1.7 for the groups $G(d, 1, r)$, which, contrary to the proof in 7.3 , spares the use of explicit matrix models. We will need the following proposition.

Proposition 9.3. If $G$ is of type $G(d, 1, r)$, then, for all distinct $\rho_{1}, \rho_{2} \in \operatorname{Irr}(G)$, we have $\left(\rho_{1} \otimes \rho_{0} \mid \rho_{2}\right) \leq 1$. This also holds for exceptional groups, except for $G_{27}, G_{29}, G_{34}$, and $G_{36}=E_{7}$. For these 4 exceptional groups, we have $\left(\rho_{1} \otimes \rho_{0} \mid\right.$ $\left.\rho_{2}\right) \leq 2$ for distinct $\rho_{1}, \rho_{2} \in \operatorname{Irr}(G)$.

Proof. The proof for the exceptional groups is a case-by-case computer check. If $G$ is of type $G(d, 1, r)$ with $r \geq 2$, let $H$ denote its natural parabolic subgroup of type $G(d, 1, r-1)$, and $\rho_{1} \in \operatorname{Irr}(G)$. Then $\left(\operatorname{Ind}_{H}^{G} 1_{G} \mid \rho_{0}\right)=\left(1_{G} \mid \operatorname{Res}_{H} \rho_{0}\right)=1$ because $H$ is a maximal parabolic subgroup. It follows that $\rho_{1} \otimes \rho_{0}$ embeds in $\rho_{1} \otimes \operatorname{Ind}_{H}^{G} 1_{G} \simeq \operatorname{Ind}_{H}^{G} \operatorname{Res}_{H} \rho_{1}$. In particular, for $\rho_{2} \in \operatorname{Irr}(G)$ we have

$$
\left(\rho_{1} \otimes \rho_{0} \mid \rho_{2}\right) \leqslant\left(\operatorname{Ind}_{H}^{G} \operatorname{Res}_{H} \rho_{1} \mid \rho_{2}\right)=\left(\operatorname{Res}_{H} \rho_{1} \mid \operatorname{Res}_{H} \rho_{2}\right)
$$


so it is sufficient to check that, if $\rho_{1} \neq \rho_{2}$, then $\left(\operatorname{Res}_{H} \rho_{1} \mid \operatorname{Res}_{H} \rho_{2}\right) \leq 1$. This is obvious considering the branching rule (see [Ze, p. 104]).

Proof of Theorem 1.7 for $G(d, 1, r)$ and exceptional groups distinct from $G_{27}, G_{29}$ or $G_{34}$. We already proved that there exists $\tilde{\iota}: \Gamma \rightarrow \operatorname{Aut}(G)$ such that

(i) $\forall \rho \in \operatorname{Irr}(G), \gamma \circ \rho \simeq \rho \circ \bar{\iota}(\gamma)$;

(ii) $\rho_{0}$ admits a model such that $\gamma \circ \rho_{0}=\rho_{0} \circ \tilde{\iota}(\gamma)$.

We want to show that all $\rho \in \operatorname{Irr}(G)$ admit an $\tilde{\imath}$-equivariant model over $K$, that is, a model over $K$ such that $\gamma \circ \rho=\rho \circ \tilde{\iota}(\gamma)$ for all $\gamma \in \Gamma$. This obviously holds if $G$ is a Weyl group, so we may assume that $G$ is not $G_{36}=E_{7}$. First note that every $\rho \in \operatorname{Irr}(G)$ embeds in some $\rho_{0}^{\otimes n}$ for some $n$, because $\rho_{0}$ is a faithful representation of $G$ (see [FH, problem 2.37]). It follows that we can define the level $N(\rho) \in \mathbb{N}$ of $\rho$ by

$$
N(\rho)=\min \left\{n \in \mathbb{N} \mid \rho \hookrightarrow \rho_{0}^{\otimes n}\right\} .
$$

In particular, $N(\rho)=m+1$ implies that there exists $\rho^{\prime} \in \operatorname{Irr}(G)$ with $N\left(\rho^{\prime}\right)=m$ such that $\rho$ embeds in $\rho^{\prime} \otimes \rho_{0}$. By (ii) we know that the representations of level at most 1 , that is the trivial representation and $\rho_{0}$, admit an equivariant model. We proceed by induction on the level. Assume that all representations of level $m$ admit an equivariant model, and let $\rho \in \operatorname{Irr}(G)$ such that $N(\rho)=m+1$. Let $\rho^{\prime} \in \operatorname{Irr}(G)$ of level $m$ such that $\rho \hookrightarrow \rho^{\prime} \otimes \rho_{0}$. By Proposition 9.3 we have $\left(\rho \mid \rho^{\prime} \otimes \rho_{0}\right)=1$. Moreover, $\rho^{\prime} \otimes \rho_{0}$ admits an equivariant model over $K$ because $\rho^{\prime}$ and $\rho_{0}$ do. Then Lemma 9.1 implies that $\rho$ admits an equivariant model over $K$ and we conclude by induction on the level.

Proof of Theorem 1.7 for $G_{27}, G_{29}, G_{34}$. Let $\mathcal{E} \subset \operatorname{Irr}(G)$ denote the set of exceptional (rational) representations described in the introduction. We note that $1_{G}, \rho_{0} \notin \mathcal{E}$. Let us consider the following algorithm.

Algorithm. (i) $L \leftarrow\left\{1_{G}, \rho_{0}\right\}$.

(ii) For all $\rho^{\prime} \in L, L^{\prime} \leftarrow L \cup\left\{\rho \in \operatorname{Irr}(G) \backslash \mathcal{E} \mid\left(\rho^{\prime} \otimes \rho_{0} \mid \rho\right)=1\right\}$.

(iii) If $\# L^{\prime}>\# L$, then $L \leftarrow L^{\prime}$ and go to (ii).

(iv) Return $L$.

The same arguments as above, based on Lemma 9.1, show that all representations in the subset $L$ of $\operatorname{Irr}(G) \backslash \mathcal{E}$ returned by this algorithm admit an equivariant model over $K$. Note that this algorithm only uses the character table of $G$. It is then enough to check that it returns $\operatorname{Irr}(G) \backslash \mathcal{E}$ for $G_{27}, G_{29}$ and $G_{34}$ in order to conclude the proof of the theorem. Indeed, this is the case if one applies step (ii) five times for $G_{27}$ and $G_{34}$, and four times for $G_{29}$.

9.3. The exceptional case of $G_{22}$. In this case we have $K=K^{\prime}=\mathbb{Q}(\mathrm{i}, \sqrt{5})$. We start from the following model $\rho: G \hookrightarrow \operatorname{GL}(V)$ over the degree 2 extension $K^{\prime \prime}=\mathbb{Q}\left(\zeta_{20}\right)$ of $K$. This model is $\operatorname{Gal}\left(K^{\prime \prime} / \mathbb{Q}\right)$-globally invariant:

$$
s \mapsto \frac{\zeta_{5}^{2}-\zeta_{5}}{\zeta_{20} \sqrt{5}}\left(\begin{array}{cc}
1 & \frac{1+\sqrt{5}}{2} \\
\frac{1+\sqrt{5}}{2} & -1
\end{array}\right), \quad t \mapsto\left(\begin{array}{cc}
0 & \zeta_{20}^{9} \\
-\zeta_{20} & 0
\end{array}\right), \quad u \mapsto\left(\begin{array}{cc}
0 & -\zeta_{20} \\
\zeta_{20}^{9} & 0
\end{array}\right) .
$$

Note that the projection $\operatorname{Gal}\left(K^{\prime \prime} / \mathbb{Q}\right) \rightarrow \operatorname{Gal}(K / \mathbb{Q})$ is not split. We check this by noting that $\operatorname{Gal}\left(K^{\prime \prime} / \mathbb{Q}\right) \simeq \mathbb{Z} / 4 \times \mathbb{Z} / 2$ admits only one subgroup isomorphic to $\operatorname{Gal}(K / \mathbb{Q}) \simeq \mathbb{Z} / 2 \times \mathbb{Z} / 2$, namely $\operatorname{Gal}\left(K^{\prime \prime} / \mathbb{Q}(\sqrt{5})\right)$, and this subgroup does not surject on $\operatorname{Gal}(K / \mathbb{Q})$, because $\sqrt{5} \in K$. 
As predicted by the theorem of Benard and Bessis, we manage to find a model $\rho_{0}$ over $K$ by Galois descent. It is possible to get a model over $K$ such that $\mathfrak{s}_{-1} \circ \rho=\rho \circ \tilde{l}\left(\mathfrak{s}_{-1}\right)$ or such that $\mathfrak{s}_{7} \circ \rho=\rho \circ \tilde{\iota}\left(\mathfrak{s}_{7}\right)$, but not both. The reason is that it is not possible to get a model such that $\mathfrak{s}_{13} \circ \rho=\rho \circ \tilde{\iota}\left(\mathfrak{s}_{13}\right)$.

Lemma 9.4. $\rho$ does not admit any model over $K$ which is globally invariant by $\operatorname{Gal}(K / \mathbb{Q}(\mathrm{i}))$.

Proof. We assume by contradiction that we are given such a model $\rho_{1}$. Since $\rho_{1}$ is faithful there exists $a \in \operatorname{Aut}(G)$ of order 2 such that $\rho_{1} \circ a=\mathfrak{s}_{13} \circ \rho_{1}$. We will see in Table 3 that $G$ has no non-trivial central automorphisms, thus all automorphisms of $G$ preserve the set of reflections. Thus we have an action of $\operatorname{Out}(G)$ on the set of faithful reflection characters, which is transitive by Theorems 5.1 and 1.4 . This action is, moreover, faithful because the group $\bar{N}$ defined in $\oint_{3}$ is trivial for $G_{22}$. It follows that $a$ and $\tilde{\iota}\left(\mathfrak{s}_{13}\right)$ have the same image in $\operatorname{Out}(G)$, namely $\bar{\iota}\left(\mathfrak{s}_{13}\right)$. In particular, $a=\tilde{\iota}\left(\mathfrak{s}_{13}\right) \circ \operatorname{Ad}(h)$ for some $h \in G$. Exhausting all possibilities for $h$ (there are only $60=\# G / Z G$ of them), we find using $\rho_{0}$ that the image of the cocycle defined by $a$ in $H^{1}\left(\operatorname{Gal}(K / \mathbb{Q}(\mathrm{i})), \mathrm{PGL}_{2}(K)\right) \simeq K^{\times} / N\left(K^{\times}\right)$is always the class of $1 / 3$ modulo $N\left(K^{\times}\right)$. Using MAGMA we check that this class is non-trivial, thus leading to a contradiction.

We now investigate which representations of $G$ admit a globally invariant model over $K$. This group has 18 irreducible representations, including two that are 1dimensional. The four 2-dimensional (reflection) representations are deduced from each other through Galois action, so none of them admits a globally invariant model over $K$. In addition to these, there are 2 faithful 4-dimensional representations and 2 faithful 6-dimensional representations.

The center of $G_{22}$ is cyclic of order 4 , generated by $Z=(s t u)^{5}$. We have $Z^{2}=(t s)^{5}$. The other representations have for kernel a non-zero subgroup of $Z(G)$.

The 8 odd-dimensional representations admit $\tilde{\imath}$-equivariant models over $K$ by Proposition 8.1, using the argument of Proposition 8.2.

A computer check shows that every non-faithful irreducible representation appears with multiplicity 1 in some tensor product $\rho_{1} \otimes \rho_{2}$, where $\rho_{1}$ and $\rho_{2}$ are odd-dimensional irreducible representations. It follows that all of these admit $\tilde{\iota}^{-}$ equivariant models.

We now study in some detail the fate of the remaining (faithful) irreducible representations. In particular, we prove the following.

Proposition 9.5. Let $\rho$ be one of the faithful 4-dimensional or 6-dimensional representations of $G_{22}$. Then $\rho$ admits globally invariant models over $K$, which induce morphisms $j_{\rho}: \Gamma \rightarrow \operatorname{Aut}(G)$. These morphisms can be chosen injective, however, the induced morphisms $\overline{\bar{j}_{\rho}}: \Gamma \rightarrow \operatorname{Out}(G)$ are never injective.

In both dimensions, one of the representations considered here satisfies $\rho(Z)=$ $-i$. It is sufficient to consider these to prove the proposition, since they are conjugated in pairs by the Galois action. We let $N=\operatorname{dim} \rho \in\{4,6\}$.

Since $\rho$ is faithful, the existence of a globally invariant model for $\rho$ leads to a morphism $j: \Gamma \rightarrow \operatorname{Aut}(G)$ satisfying $\gamma \circ \rho \simeq \rho \circ j(\gamma)$ for all $\gamma \in \Gamma$, such that the associated cocycle $J=\left\{\gamma \mapsto J_{\gamma}\right\}$ is cohomologically trivial.

We use the following procedure to check the proposition for an arbitrary $j$ : $\Gamma \rightarrow \operatorname{Aut}(G)$ satisfying $\gamma \circ \rho \simeq \rho \circ j(\gamma)$ for all $\gamma \in \Gamma$. Let $\gamma_{0}=\mathfrak{s}_{13}$ and note that 
$K^{\gamma_{0}}=\mathbb{Q}(\mathrm{i})$. Recall that $\gamma_{0}$ and $\mathfrak{c}$ generate $\Gamma=\operatorname{Gal}(\mathbb{Q}(\mathrm{i}, \sqrt{5}) / \mathbb{Q})$. Restricting $J$ to $\left\langle\gamma_{0}\right\rangle$ yields a class in $\mathbb{Q}(\mathrm{i})^{\times} / N K^{\times}$where $N(x)=x \gamma_{0}(x)$. If this class is non-zero, then $J$ cannot be cohomologically trivial; otherwise there exists $M \in \mathrm{GL}_{N}(K)$, which can be explicitly determined, such that $J_{\gamma_{0}}=M^{-1} \gamma_{0}(M)$ in $\operatorname{PGL}_{N}(K)$. Then the cocycle $\gamma \mapsto Q_{\gamma}=M J_{\gamma} \gamma(M)^{-1}$ is cohomologous to $J$ and $Q_{\gamma_{0}}=1$. Since

$$
\gamma_{0}\left(Q_{\mathfrak{c}}\right)=Q_{\gamma_{0}} \gamma_{0}\left(Q_{\mathfrak{c}}\right)=Q_{\gamma_{0} \mathfrak{c}}=Q_{\mathfrak{c} \gamma_{0}}=Q_{\mathfrak{c}} \mathfrak{c}\left(Q_{\gamma_{0}}\right)=Q_{\mathfrak{c}}
$$

it follows that $\gamma_{0}\left(Q_{\mathfrak{c}}\right)=Q_{\mathfrak{c}}$, i.e., $Q_{\mathfrak{c}} \in \mathrm{PGL}_{N}(K)^{\gamma_{0}}=\operatorname{PGL}_{N}\left(K^{\gamma_{0}}\right)$, and that $Q_{\gamma_{0} \mathfrak{c}}=Q_{\mathfrak{c}}$. In other words, $Q$ belongs to the image of the inflation map

$$
Z^{1}\left(\operatorname{Gal}\left(K^{\gamma_{0}} / \mathbb{Q}\right), \operatorname{PGL}_{N}\left(K^{\gamma_{0}}\right)\right) \rightarrow Z^{1}\left(\Gamma, P G L_{N}(K)\right) .
$$

Since the inflation map between Brauer groups is injective this map is also injective and we are again reduced to a cyclic case, hence to a norm equation solvable with MAGMA.

Using CHEVIE we get all such morphisms. The list obtained does not depend on whether $\rho$ has dimension 4 or 6 . If $N=4$, the morphisms $j$ such that $J$ is cohomologous to 0 all satisfy $\overline{j(\mathfrak{c})}=\overline{j\left(\mathfrak{s}_{7}\right)}=\bar{\iota}\left(\mathfrak{s}_{7}\right)$ and $\overline{j\left(\mathfrak{s}_{13}\right)}=\bar{\iota}(1)$. Some of these morphisms $j$ are injective.

In case $N=6$, the morphisms $j$ such that $J$ is cohomologically trivial are all injective, and again send $\mathfrak{s}_{13}$ to an inner automorphism. Moreover, $\overline{j(\mathfrak{c})} \in$ $\left\{\bar{l}(\mathfrak{c}), \bar{\imath}\left(\mathfrak{s}_{7}\right)\right\}$ and the two possibilities occur.

The choice of any morphism such that $J$ is cohomologically trivial leads to a globally invariant model for the representations. One of these morphisms, such that $J$ is cohomologically trivial for both representations, has the following simple form: $j\left(\mathfrak{s}_{13}\right)=\operatorname{Ad}(t)$ and $j(\mathfrak{c})$ sends $(s, t, u)$ to $\left(u^{t u}, t, s^{t s t}\right)$.

Remark 9.6. Using the results proved here, one gets a simpler proof of the theorem of Benard and Bessis. First consider non-dihedral $G(d e, e, r)$. Since all irreducible representations of the groups $G(d e, e, r)$ appear as a multiplicity 1 component of some representation of $G(d e, 1, r)$, we are reduced by Lemma 9.1 to the case of $G(d, 1, r)$, then to its reflection representation by Proposition 9.3 , and we know that this representation is defined over $K$ by the very definition of these groups, or by [Bens, 7.1.1] which apply to all cases. The case of the dihedral groups is classical (their representations are mostly reflection representations). Most exceptional groups are then dealt with by Proposition 9.3. Finally, the algorithm used here for the few remaining ones completes the proof, and is more efficient than the one used in $[\mathrm{Bes}$.

\section{ON THE MORPHISMS $\bar{\iota}_{\chi}$}

We first show how to derive Corollary 1.5, stated for arbitrary reflection groups, from Theorem 1.4 It is an obvious consequence of the following proposition.

Proposition 10.1. Let $G$ be any finite complex reflection group, let $K$ be its field of definition and let $\Gamma=\operatorname{Gal}(K / \mathbb{Q})$. Let $\mathcal{S}$ be the set of irreducible characters of $G$ which cannot be realized over $\mathbb{Q}$. Then for any $\chi \in \mathcal{S}$ there exists an injection $\Gamma \stackrel{\bar{\iota}_{\chi}}{\longrightarrow} \operatorname{Out}(G)$ such that for any $\gamma \in \Gamma \gamma(\chi)=\chi \circ \bar{\iota}_{\chi}(\gamma)$.

Proof. Proposition 10.1 is an obvious consequence of Theorem 1.4 when $G$ is irreducible. For a general reflection group $G$, we have a decomposition into irreducible groups: $V=V_{1} \oplus \cdots \oplus V_{n}, G=G_{1} \times \cdots \times G_{n}$ where $G_{i} \subset \operatorname{GL}\left(V_{i}\right)$. If $K_{i}$ is 
the field of definition of $G_{i}$, then $K$ is the subfield of $\mathbb{C}$ generated by $K_{1}, \ldots, K_{n}$, whence if $\Gamma_{i}=\operatorname{Gal}\left(K_{i} / \mathbb{Q}\right)$ we have natural quotient morphisms $\Gamma \rightarrow \Gamma_{i}$ such that the product map $\Gamma \rightarrow \prod_{i} \Gamma_{i}$ is injective. We deduce an injective map $\Gamma \rightarrow \operatorname{Out}(G)$ by composing $\Gamma \rightarrow \prod_{i} \Gamma_{i}$ with the individual maps $\Gamma_{i} \rightarrow \operatorname{Out}\left(G_{i}\right)$ deduced from Theorem 1.4 and then with the natural injection $\operatorname{Out}\left(G_{1}\right) \times \ldots \operatorname{Out}\left(G_{n}\right) \rightarrow \operatorname{Out}(G)$. Since any irreducible character $\chi$ of $G$ is a product of irreducible characters of the $G_{i}$, Proposition 10.1 follows readily.

We now study further the structure of $\operatorname{Out}(G)$, w.r.t. the morphisms $\bar{\iota}_{V}: \Gamma \rightarrow$ $\operatorname{Out}(G)$. We now assume that $G$ is irreducible. Recall that, when $G \neq \mathfrak{S}_{6}$, we have $\operatorname{Out}(G)=\bar{C} \cdot \bar{A}$, as a direct consequence of Theorem 1.1, and $\bar{A}=\bar{N} \rtimes \Gamma$. Moreover, it is clear that $\bar{N}$ normalizes $\bar{C}$, and centralizes $\bar{C}$ when $\bar{N}$ acts trivially on the linear characters.

The next lemma is the cornerstone of the interaction between $\bar{\iota}_{V}(\Gamma)$ and $\bar{C}$.

Lemma 10.2. Let $G$ be an irreducible complex reflection group. Then $\chi\left(\bar{\iota}_{V}(\gamma)(g)\right)$ $=\gamma(\chi(g))$ for all linear characters $\chi$ and all $\gamma \in \Gamma$.

Proof. The lemma results from the fact that $\bar{\iota}_{\chi}$ can be chosen to be the same for $\chi_{V}$ and for the linear characters. This is clear from Theorem 1.4 since either $\bar{\iota}_{\chi}$ can be chosen independent of $\chi$, or we are in a $G(d e, e, r)$, and the action of $\bar{N}$ is trivial on both $V$ and the linear characters, since they are the restriction from $G(d e, 1, r)$ of a character which does not split.

Proposition 10.3. $\bar{\iota}_{V}(\Gamma) \subset \bar{A}$ commutes with $\bar{C}$. In particular, $\bar{A}$ commutes with $\bar{C}$ as soon as $\bar{N}=\{1\}$.

Proof. By Theorems 1.6 and 1.7 there exists an extension $K^{\prime \prime}$ of $K$, a $\mathbb{Q}$-form of $V \otimes_{K} K^{\prime \prime}$ and a map $\iota: \operatorname{Gal}\left(K^{\prime \prime} / \mathbb{Q}\right) \rightarrow \operatorname{Aut}(G)$ such that for $g \in G \subset \operatorname{GL}(V)$ and $\gamma \in \operatorname{Gal}\left(K^{\prime \prime} / \mathbb{Q}\right)$ we have $\gamma(g)=\iota(\gamma)(g)$, which induce $\bar{\iota}_{V}: \operatorname{Gal}(K / \mathbb{Q}) \rightarrow \operatorname{Out}(G)$, except possibly for $G_{22}$. In the case of $G_{22}$, from the globally invariant model over $K^{\prime \prime}$ we get a morphism $\iota: \operatorname{Gal}\left(K^{\prime \prime} / \mathbb{Q}\right) \rightarrow \operatorname{Aut}(G)$. The induced morphism $j$ to $\operatorname{Out}(G)$ factors through $\operatorname{Gal}(K / \mathbb{Q})$, as $\gamma \circ \chi_{V}=\chi_{V}$ for $\gamma \in \operatorname{Gal}\left(K^{\prime \prime} / K\right)$, and $\bar{N}=1$ in this case. It then coincides with $\bar{\iota}_{V}: \operatorname{Gal}(K / \mathbb{Q}) \rightarrow \operatorname{Out}(G)$, as $\chi_{V} \circ j(\gamma)=\chi_{V} \circ \bar{\iota}_{V}(\gamma)$ for all $\gamma \in \Gamma$, hence $j(\gamma) \bar{\iota}_{V}(\gamma)^{-1} \in \bar{N}=\{1\}$.

Let $\alpha_{\chi} \in C$ a central automorphism defined by $\alpha_{\chi}(g)=g \chi(g)^{-1}$, where $\chi$ is a morphism $G \rightarrow Z G$, or equivalently, a linear character whose image lies in $Z G$ (see the paragraph above Lemma 12.4). It follows that $\iota(\gamma) \circ \alpha_{\chi} \circ \iota(\gamma)^{-1}(g)=$ $\iota(\gamma)\left(\iota(\gamma)^{-1}(g) \chi\left(\iota(\gamma)^{-1}(g)\right)^{-1}\right)=g \iota(\gamma)\left(\chi\left(\iota(\gamma)^{-1}(g)\right)^{-1}\right)$. Now $\chi \circ \iota(\gamma)^{-1}=\chi \circ$ $\bar{\iota}_{V}(\gamma)^{-1}=\gamma^{-1} \circ \chi$ by Lemma 10.2, and $\iota(\gamma)\left(\chi\left(\iota(\gamma)^{-1}(g)\right)^{-1}\right)=\gamma\left(\chi\left(\iota(\gamma)^{-1}(g)\right)^{-1}\right)$ as $\chi\left(\iota(\gamma)^{-1}(g)\right) \in Z G \subset G$. It follows that $\iota(\gamma)\left(\chi\left(\iota(\gamma)^{-1}(g)\right)^{-1}\right)=\chi(g)^{-1}$ hence $\iota(\gamma) \circ \alpha_{\chi} \circ \iota(\gamma)^{-1}(g)=\alpha_{\chi}(g)$ whence $\bar{\iota}_{V}(\Gamma)$ commutes with $C$.

\section{INVARIANTS}

We prove Corollary 1.8, whose statement we recall. We denote by $V^{*}$ the dual of a vector space $V$.

Proposition 11.1. Let $G \subset \mathrm{GL}(V)$ be an irreducible complex reflection group where $V$ is a $K^{\prime \prime}$-vector space, with $K^{\prime \prime}$ as in the introduction. There is a $\mathbb{Q}$-form $V=V_{0} \otimes_{\mathbb{Q}} K^{\prime \prime}$ such that the fundamental invariants of $G$ can be taken rational, i.e., in the symmetric algebra $S\left(V_{0}^{*}\right)$. 
Proof. By Theorem 1.7 and the matrix model over $K^{\prime \prime}$ exhibited for $G_{22}$ in section 9.3 , we may assume that $G \subset \mathrm{GL}\left(V_{0} \otimes_{\mathbb{Q}} K^{\prime \prime}\right)$ is globally invariant by $\Gamma=$ $\operatorname{Gal}\left(K^{\prime \prime} / \mathbb{Q}\right)$. Let $f_{1}, \ldots, f_{r}$ (where $r=\operatorname{dim} V$ ) be fundamental invariants, i.e., algebraically independent polynomials such that $S\left(V^{*}\right)^{G}=K^{\prime \prime}\left[f_{1}, \ldots, f_{n}\right]$. We want to find algebraically independent $g_{1}, \ldots, g_{r} \in S\left(V_{0}^{*}\right)$ such that we still have $S\left(V^{*}\right)^{G}=K^{\prime \prime}\left[g_{1}, \ldots, g_{n}\right]$. Our strategy will be as follows: we will set $g_{i}=$ $\sum_{\gamma \in \Gamma} \gamma\left(\lambda f_{i}\right)$ where $\lambda \in K^{\prime \prime}$. Then the $g_{i}$ are still invariant since for $g \in G$ we have $g\left(g_{i}\right)=\sum_{\gamma \in \Gamma} \gamma\left(\lambda \gamma^{-1}(g) f_{i}\right)=\sum_{\gamma \in \Gamma} \gamma\left(\lambda f_{i}\right)$, the last equality since $\gamma^{-1}(g) \in G$.

It is thus sufficient to show that we may choose $\lambda$ such that $g_{i}$ are still algebraically independent. By the Jacobian criterion, it is enough to show that we may choose $\lambda$ such that, if $x_{1}, \ldots, x_{r}$ is a basis of $V_{0}^{*}$, so that $S\left(V^{*}\right) \simeq K^{\prime \prime}\left[x_{1}, \ldots, x_{r}\right]$, we have $\operatorname{det}\left(\frac{\partial g_{i}}{\partial x_{j}}\right)_{i, j} \neq 0$.

We use the following version of the algebraic independence of automorphisms:

Proposition 11.2 (Bbk1, Chap. V, $\S 10$, Théorème 4]). Let $Q$ be an infinite field, let $K$ be a finite Galois extension of $Q$ with Galois group $\Gamma$, let $\Omega$ be an arbitrary extension of $K$ and let $\left\{X_{\gamma}\right\}_{\gamma \in \Gamma}$ be indeterminates indexed by the elements of $\Gamma$. Let $F \in \Omega\left[X_{\gamma}\right]_{\gamma \in \Gamma}$ be a polynomial such that $F\left((\gamma(x))_{\gamma \in \Gamma}\right)=0$ for any $x \in K$. Then $F=0$.

We apply the proposition with $Q=\mathbb{Q}, \Omega=K^{\prime \prime}$ and $F=\operatorname{det}\left(\sum_{\gamma \in \Gamma} X_{\gamma} \gamma\left(\frac{\partial f_{i}}{\partial x_{j}}\right)\right)_{i, j}$. The polynomial $F$ evaluated at $X_{1}=1$ and $X_{\gamma}=0$ for $\gamma \neq 1$ is equal to $\operatorname{det}\left(\frac{\partial f_{i}}{\partial x_{j}}\right)_{i, j}$ which is non-zero, so $F$ is non-zero. By the theorem, there exists $\lambda \in K^{\prime \prime}$ such that $F\left((\gamma(\lambda))_{\gamma}\right) \neq 0$. But $F\left((\gamma(\lambda))_{\gamma}\right)=\operatorname{det}\left(\frac{\partial g_{i}}{\partial x_{j}}\right)_{i, j}$.

We now prove Corollary 1.9 whose statement we recall.

Proposition 11.3. The morphism $V^{\mathrm{reg}} \rightarrow V^{\mathrm{reg}} / G$ is defined over $\mathbb{Q}$.

Proof. We use the notations of the previous proof, in particular, we assume that $G \subset \mathrm{GL}\left(V_{0} \otimes_{\mathbb{Q}} K^{\prime \prime}\right)$ is globally invariant by $\Gamma=\operatorname{Gal}\left(K^{\prime \prime} / \mathbb{Q}\right)$.

Let $\mathcal{H}$ be the set of reflecting hyperplanes for $G$ and for each $H \in \mathcal{H}$, let $l_{H}$ be a linear form defining $H$. For $H \in \mathcal{H}$ let $e_{H}$ be the order of the subgroup of $G$ fixing $H$, and let $\Delta=\prod_{H \in \mathcal{H}} l_{H}^{e_{H}}$. It is well known that $\Delta \in S\left(V^{*}\right)^{G}$ (see e.g., OT, 6.44]). Thus the variety $V^{\text {reg }} / G$ is the open subvariety of $\operatorname{Spec}\left(K^{\prime \prime}\left[f_{1}, \ldots, f_{n}\right]\right)$ whose function ring is the localization by the principal ideal $\Delta$. It is thus enough to show that we may choose the $f_{i}$ such that a multiple of $\Delta$ belongs to $\mathbb{Q}\left[f_{1}, \ldots, f_{n}\right]$. If, as in the previous proposition, we choose $f_{i} \in S\left(V_{0}^{*}\right)^{G}$, it will be enough to show that we have a multiple of $\Delta$ in $S\left(V_{0}^{*}\right)$ since $S\left(V_{0}^{*}\right) \cap S\left(V^{*}\right)^{G}=S\left(V_{0}^{*}\right)^{G}$. Since $\mathcal{H}$ is globally invariant by $\Gamma$, for any $\gamma \in \Gamma$ there exists $\lambda_{\gamma} \in K^{\prime \prime}$ such that $\gamma(\Delta)=\lambda_{\gamma} \Delta$, and it is clear that $\left\{\gamma \mapsto \lambda_{\gamma}\right\} \in Z^{1}\left(\Gamma, K^{\prime \prime \times}\right)$. By Hilbert's Theorem 90 we have that $H^{1}\left(\Gamma, K^{\prime \prime} \times\right)$ is trivial, thus $\left\{\gamma \mapsto \lambda_{\gamma}\right\}$ is a coboundary, i.e., there exists $\lambda \in K^{\prime \prime \times}$ such that $\lambda_{\gamma}=\lambda^{-1} \gamma(\lambda)$. We then get that $\gamma\left(\lambda^{-1} \Delta\right)=\lambda^{-1} \Delta$ for any $\gamma$, thus $\lambda^{-1} \Delta \in S\left(V_{0}^{*}\right)$.

\section{QuASI-INDECOMPOSABle GROUPS, AND NON-IRREDUCIBLE GROUPS}

In this chapter, we prove Proposition 1.10 and Theorem 1.11, and then go on to describe the non-abelian factors and the central automorphisms of irreducible complex reflection groups. 
We recall that a group is indecomposable if it has no non-trivial decomposition as a direct product. We recall the statement of the Krull-Remak-Schmidt theorem for finite groups.

Theorem 12.1 (Krull-Remak-Schmidt). Given two decompositions $G=G_{1} \times \cdots \times$ $G_{k}=H_{1} \times \cdots \times H_{k^{\prime}}$ of the finite group $G$ as a product of indecomposable factors, we have $k=k^{\prime}$ and there is a permutation $\sigma$ of $\{1, \ldots, k\}$ and a central automorphism $\alpha$ such that $\alpha\left(H_{i}\right)=G_{\sigma(i)}$.

Proof. See e.g. [Ro, 3.3.8].

12.1. Quasi-indecomposable groups. We say that a group $G$ is quasi-indecomposable if in any direct product decomposition $G=G_{1} \times G_{2}$, we have either $G_{1} \subseteq$ $Z G$ or $G_{2} \subseteq Z G$.

Proposition 12.2. Let $G$ be a finite quasi-indecomposable group. Then $G$ admits a decomposition of the form $G=Z \times \hat{G}$ where $Z \subseteq Z G$ and $\hat{G}$ is either trivial or is a non-abelian indecomposable group. In such a decomposition $\hat{G}$ (called the non-abelian factor of $G$ and denoted $\operatorname{Nab}(G)$ ) and $Z$ (called the central factor of $G)$ are unique up to isomorphism.

Proof. First, we observe that $G$ admits such a decomposition. Indeed, being finite, $G$ admits a decomposition $G=G_{1} \times \cdots \times G_{n}$ as a product of indecomposable groups. By assumption, at most one of the $G_{i}$ is non-abelian; we set $\hat{G}$ equal to that factor and $Z$ equal to the product of the other factors.

Assume now that $G$ admits two such decompositions $G=Z \times \hat{G}=Z_{1} \times \hat{G}_{1}$. If we refine the decompositions to a product of indecomposable groups, by the KrullRemak-Schmidt theorem there is an automorphism which sends one decomposition to the other, and this automorphism must send the non-abelian factor in one decomposition to the other, whence the isomorphisms between $\hat{G}$ and $\hat{G}_{1}$ and $Z$ and $Z_{1}$.

We now relate decompositions of quasi-indecomposable groups to central endomorphisms. An endomorphism $\alpha: G \rightarrow G$ is central if for any $g \in G$, we have $\alpha(g) \in g Z G$.

Lemma 12.3. Let $G$ be a finite quasi-indecomposable group, and let $\alpha$ be a central endomorphism of $G$ with a maximal kernel (that is, $\operatorname{ker} \alpha$ is a maximal element for inclusion among the $\operatorname{ker} \beta$ where $\beta$ runs over the central endomorphisms). Then $G=\operatorname{ker} \alpha \times \operatorname{Im}(\alpha)$ is a decomposition of $G$ with $\operatorname{ker} \alpha \subseteq Z G$ and $\operatorname{Im}(\alpha)$ indecomposable non-abelian (or trivial).

Proof. Since $\operatorname{ker} \alpha \subseteq \operatorname{ker} \alpha^{2}$ and $\operatorname{ker} \alpha$ is maximal, we have $\operatorname{ker} \alpha^{2}=\operatorname{ker} \alpha$, whence $\operatorname{Im}\left(\alpha^{2}\right)=\operatorname{Im}(\alpha)$ since \#G=\# ker $\alpha \# \operatorname{Im}(\alpha)$. In particular, $\alpha$ is injective on $\operatorname{Im}(\alpha)$, so $\operatorname{ker} \alpha \cap \operatorname{Im}(\alpha)=\{1\}$. Thus $G=\operatorname{ker} \alpha \times \operatorname{Im}(\alpha)$; and $\operatorname{Im}(\alpha)$ is indecomposable, otherwise if $\operatorname{Im}(\alpha)=Z \times \hat{G}$ where $Z$ is central, and if $\beta$ is the projection $\operatorname{Im}(\alpha) \rightarrow \hat{G}$, then $\beta \circ \alpha$ would be central and would have a greater kernel than $\alpha$, contradicting the maximality of ker $\alpha$.

Given a central endomorphism of $G$, we define a homomorphism $\chi_{\alpha}: G \rightarrow Z G$ by the formula $\chi_{\alpha}(g)=g \alpha\left(g^{-1}\right)$. Conversely, any homomorphism $\chi: G \rightarrow Z G$ gives rise to a central endomorphism by the formula $\alpha_{\chi}(g)=g \chi(g)^{-1}$. We have $\operatorname{ker} \alpha_{\chi}=\{z \in Z G \mid \chi(z)=z\}$. 
Lemma 12.4. Let $G$ be a quasi-indecomposable finite group with cyclic center. Then in a decomposition $G=Z \times \hat{G}$ with $Z$ central and $\hat{G}$ indecomposable nonabelian, the factor $Z$ is unique.

Proof. In view of the previous lemma, it is sufficient to prove that given two central endomorphisms $\alpha$ and $\beta$, there is a central endomorphism $\gamma$ such that ker $\alpha \subseteq \operatorname{ker} \gamma$ and $\operatorname{ker} \beta \subseteq \operatorname{ker} \gamma$. Let us take $\gamma=\alpha \circ \beta$. Then clearly $\operatorname{ker} \beta \subseteq \operatorname{ker} \gamma$, and we will have ker $\alpha \subseteq \operatorname{ker} \gamma$ if we can show that restricted to $Z G$ the endomorphisms $\alpha$ and $\beta$ commute. Now $\alpha(\beta(z))=\beta(z) \chi_{\alpha}(\beta(z))^{-1}=z \chi_{\beta}\left(z^{-1}\right) \chi_{\alpha}\left(z^{-1}\right) \chi_{\alpha}\left(\chi_{\beta}(z)\right)$, and this formula is symmetric in $\alpha$ and $\beta$ since the two endomorphisms $\chi_{\alpha}$ and $\chi_{\beta}$ of the cyclic group $Z G$ commute with each other.

Remark 12.5. Non-uniqueness of the central factor.

The group $\mathbb{Z} / 2 \times W\left(B_{2}\right)$ does not have a unique central factor. We will see that $W\left(B_{2}\right)$ is indecomposable, so the above is a decomposition into a central and a non-abelian indecomposable factor. If $\{1, \varepsilon\}$ are the two elements of the factor $\mathbb{Z} / 2$ and $\left\{1, w_{0}\right\}$ the two elements of $Z\left(W\left(B_{2}\right)\right)$, then $\left\{1, \varepsilon w_{0}\right\}$ is another direct factor of $W\left(B_{2}\right)$.

Remark 12.6. Non-uniqueness of the non-abelian factor.

Even if the central factor is unique, $\operatorname{Nab}(G)$ may not be. We will see that $W\left(G_{2}\right)$ is quasi-indecomposable. Having a cyclic center, it has a unique central factor which is the center $\left\{1, w_{0}\right\}$. A possible non-abelian factor is $W\left(A_{2}\right) \subset W\left(G_{2}\right)$. However, this is not the only one. If we consider the sign character $x \mapsto \operatorname{sgn}(x)$ of $W\left(A_{2}\right)$ to take its values in $\left\{1, w_{0}\right\}$, then the subgroup formed of $\left\{x \operatorname{sgn}(x) \mid x \in W\left(A_{2}\right)\right\}$ is another possible non-abelian factor.

We will see, however, that both factors are unique for most irreducible complex reflection groups. We prove now Proposition 1.10

Proposition 12.7. Irreducible finite complex reflection groups are quasi-indecomposable.

Proof. We prove the result case by case, starting with the imprimitive groups $G(d e, e, r)$ of rank $r \geq 3$. Recall that such a group is of the form $G=D \rtimes \mathfrak{S}_{r}$, where $D$ is the subgroup of diagonal matrices in $G$. Let $\pi$ be the quotient map $G \rightarrow \mathfrak{S}_{r}$ and consider a decomposition $G=G_{1} \times G_{2}$. Then we get $\mathfrak{S}_{r}=\pi\left(G_{1}\right) \pi\left(G_{2}\right)$, a product of two normal subgroups. Since any proper normal subgroup of $\mathfrak{S}_{r}$ is in $\mathfrak{A}_{r}$, we must have, e.g., $\pi\left(G_{1}\right)=\mathfrak{S}_{r}$. Since $\pi\left(G_{2}\right)$ commutes with $\pi\left(G_{1}\right)$ and the center of $\mathfrak{S}_{r}$ is trivial (since $r \geq 3$ ) we have $\pi\left(G_{2}\right)=1$, i.e., $G_{2} \subseteq D$. Further, the action of $\pi\left(G_{1}\right)=\mathfrak{S}_{r}$ on $G_{2}$ is trivial, so $G_{2} \subseteq Z G$.

To treat the other cases, we will follow the arguments of [M1, 6.2, lemme 2]. Assume that $G \subset \mathrm{GL}(V)$, an irreducible group, admits a decomposition $G_{1} \times G_{2}$ where neither of the $G_{i}$ is abelian. Since an irreducible representation of a product is the tensor product of irreducible representations of the factors, we must have $V=$ $V_{1} \otimes V_{2}$ where $V_{i}$ affords a faithful irreducible representation of $G_{i}$. In particular, we must have $\operatorname{dim} V_{i}>1$ since $G_{i}$ is non-abelian.

These remarks immediately solve the cases of $G(d e, e, 2)$ or more generally the irreducible groups of prime rank. It remains to consider the exceptional groups of rank 4,6 or 8. For that, we use that if $G$ is not quasi-indecomposable, then $V \otimes V \simeq V_{1} \otimes V_{1} \otimes V_{2} \otimes V_{2}=\left(S^{2} V_{1} \oplus \Lambda^{2} V_{1}\right) \otimes\left(S^{2} V_{2} \oplus \Lambda^{2} V_{2}\right)$ has at least 4 irreducible 
components (since $\operatorname{dim} V_{i}>1$ implies that the decomposition $V_{i} \otimes V_{i}=S^{2} V_{i} \oplus \Lambda^{2} V_{i}$ is non-trivial).

Now it is a theorem of Steinberg [Bbk2, Exercise 3] that for an irreducible complex reflection group, $\Lambda^{2} V$ is irreducible. Thus, we see that if $S^{2} V$ has less than 3 irreducible components, then $V \otimes V=S^{2} V \oplus \Lambda^{2} V$ will have less than 4, and thus $G$ will be quasi-indecomposable. When $G$ is real (a Coxeter group) the representation $S^{2} V$ contains the trivial representation. It turns out that for any exceptional irreducible complex reflection group (not only those of rank 4, 6 or 8), $S^{2} V$ is irreducible when $G$ is not real and $S^{2} V-1_{G}$ is irreducible when $G$ is real (this can easily checked by computer from the character table of $G$ ).

We have the following consequence of the Krull-Remak-Schmidt theorem for direct products of quasi-indecomposable groups:

Theorem 12.8. Let $G=G_{1} \times \cdots \times G_{n}$ be a direct product of finite quasiindecomposable groups, such that for any $i, j$ we have $\operatorname{Nab}\left(G_{i}\right) \simeq \operatorname{Nab}\left(G_{j}\right) \Rightarrow$ $G_{i} \simeq G_{j}$. Then any automorphism of $G$ is of the form $\alpha \circ \beta$, where $\alpha$ is a central automorphism and $\beta$ satisfies $\beta\left(G_{i}\right)=G_{\sigma(i)}$ for some permutation $\sigma$ of $\{1, \ldots, n\}$.

Proof. Let $\gamma \in \operatorname{Aut}(G)$ and let $G=G_{1} \times \cdots \times G_{k}$ be a decomposition of $G$ in indecomposable factors. Let us apply the Krull-Remak-Schmidt theorem to the decomposition given by $H_{i}=\gamma\left(G_{i}\right)$; we get a central automorphism $\alpha$ of $G$ and $\sigma \in \mathfrak{S}_{k}$ such that $\alpha\left(G_{i}\right)=H_{\sigma^{-1}(i)}$. Letting $\beta=\alpha^{-1} \circ \gamma$ it follows that $\gamma$ can be written $\alpha \circ \beta$ with $\alpha$ a central automorphism of $G$ and $\beta\left(G_{i}\right)=G_{\sigma(i)}$ for some $\sigma \in \mathfrak{S}_{k}$.

If we lump together the product of all abelian $G_{i}$ as a single subgroup $Z$, we get that any automorphism of a group of the form $Z \times G_{1} \times \cdots \times G_{n}$ where $Z$ is abelian and the $G_{i}$ are non-abelian indecomposable groups is of the form $\alpha \circ \beta$ where $\alpha$ is central and $\beta\left(G_{i}\right)=G_{\sigma(i)}$ for some $\sigma \in \mathfrak{S}_{n}$, as $\beta\left(G_{i}\right)$, being non-abelian, cannot be mapped to one of the indecomposable factors of $Z$. Let $\beta_{Z} \in \operatorname{Aut}(G)$ being defined by $\beta_{Z}(g)=\beta(g)$ for $g \in Z$ and $\beta_{Z}(g)=g$ for $g \in G_{i}$; similarly, $\beta_{G}(g)=\beta(g)$ for $g \in G_{i}, \beta_{G}(g)=g$ for $g \in Z$. We have $\beta=\beta_{Z} \circ \beta_{G}$ and $\gamma=\left(\alpha \circ \beta_{Z}\right) \circ \beta_{G}$. Since $\beta_{Z}$ is a central automorphism, replacing $\alpha$ by $\alpha \circ \beta_{Z}$ and $\beta$ by $\beta_{G}$ we thus can assume that $\beta$ acts trivially on $Z$.

Rewriting the group $G$ of the statement as $Z_{1} \times \cdots \times Z_{n} \times \hat{G}_{1} \times \cdots \times \hat{G}_{n}$ where $Z_{i}$ are the central factors of the $G_{i}$ and $\hat{G}_{i}$ are their non-abelian factors, we get that any automorphism of $G$ is of the form $\alpha \circ \beta$ where $\alpha$ is central and $\beta$ is trivial on $Z_{1} \times \cdots \times$ $Z_{n}$ and effects a permutation $\sigma$ of the $\hat{G}_{i}$. The assumption of the statement that $\operatorname{Nab}\left(G_{i}\right) \simeq \operatorname{Nab}\left(G_{j}\right) \Rightarrow G_{i} \simeq G_{j}$ now implies that $G_{\sigma(i)} \simeq G_{i}$, hence by Proposition 12.2 there exists isomorphisms $\delta_{i}: Z_{\sigma(i)} \rightarrow Z_{i}$ between the corresponding central factors. We recall that $\beta$ acts trivially on each $Z_{i}$, and introduce $\delta \in \operatorname{Aut}(G)$ such that $\delta(g)=g$ for $g \in \hat{G}_{i}$ and $\delta(g)=\delta_{i}(g)$ for $g \in Z_{i}$. Letting $\beta^{\prime}=\delta^{-1} \circ \beta$ we have $\beta^{\prime}(g)=\beta(g) \in \hat{G}_{\sigma(i)}$ for $g \in \hat{G}_{i}$ and $\beta^{\prime}(g)=\delta_{i}^{-1}(g) \in Z_{\sigma(i)}$ for $g \in G_{i}$. Since $\delta$ is clearly central we get $\gamma=\alpha^{\prime} \circ \beta^{\prime}$, with $\alpha^{\prime}=\alpha \circ \delta$ a central automorphism and $\beta^{\prime}\left(G_{i}\right)=G_{\sigma(i)}$, which concludes the proof.

We now prove Theorem 1.11.

Theorem 12.9. Let $G \subset \mathrm{GL}(V)$ be a complex reflection group and let $G=$ $G_{1} \times \cdots \times G_{n}$ be its decomposition in irreducible factors. Then the following are equivalent: 
(i) $N o G_{i}$ is isomorphic to $\mathfrak{S}_{6}$, and for any $i, j$, we have $\operatorname{Nab}\left(G_{i}\right) \simeq \operatorname{Nab}\left(G_{j}\right) \Rightarrow$ $G_{i} \simeq G_{j}$.

(ii) Any automorphism of $G$ is of the form $\alpha \circ \beta \circ \gamma$, where $\alpha$ is central, $\beta$ is induced by $N_{\mathrm{GL}(V)}(G)$, and $\gamma$ stabilizes the $G_{i}$ and preserve their reflections.

Moreover, for every complex reflection group $G$, any automorphism which preserve the reflections can be written as $\beta \circ \gamma$, with $\beta, \gamma$ as above.

Proof. We first prove the last assertion of the theorem. An automorphism which maps reflections to reflections necessarily permutes the factors $G_{i}$. This can be seen for instance by considering the graph whose vertices are the set of reflections, with an edge between two reflections $s, u$ whenever $s u \neq u s$ or $s$ is a power of $u$; then the connected components of this graph are in natural one-to-one correspondence with the factors $G_{i}$, and our automorphism induces an automorphism of the graph, which permutes its connected components. Then, since a permutation of isomorphic $G_{i}$ can be effected by an element of $N=N_{\mathrm{GL}(V)}(G)$, such an automorphism is indeed of the form $\beta \circ \gamma$ where $\beta$ is affected by an element of $N$ and $\gamma$ stabilizes the $G_{i}$ and preserves their reflections.

We now observe that (ii) $\Rightarrow$ (i) is clear, since if one of the $G_{i}$ is isomorphic to $\mathfrak{S}_{6}$ the exceptional automorphisms of $\mathfrak{S}_{6}$ are not of the form given in (ii), and, if two non-isomorphic components $G_{i}$ and $G_{j}$ have isomorphic non-abelian factors: $G_{i}=Z_{i} \times \hat{G}$ and $G_{j}=Z_{j} \times \hat{G}$, the automorphism of $G$ which exchanges the isomorphic factors $\hat{G}$ while leaving the other factors invariant is not either of the form given in (ii).

We finally prove (i) $\Rightarrow$ (ii). By Theorem 12.8, since (i) holds, any automorphism is of the form $\alpha_{1} \circ \beta_{1}$ where $\alpha_{1}$ is central and $\beta_{1}$ permutes the $G_{i}$. Since a permutation of isomorphic $G_{i}$ can be affected by an element of $N$, we can write $\beta_{1}=\beta_{2} \circ \beta_{3}$ where $\beta_{2} \in N$ and $\beta_{3}\left(G_{i}\right)=G_{i}$. Moreover, since no $G_{i}$ is isomorphic to $\mathfrak{S}_{6}$, by Theorem 1.1, $\beta_{3}$ itself is of the form $\beta_{3}=\alpha_{2} \circ \gamma$, where $\alpha_{2}$ is central and $\gamma$ preserves the reflections. Since the subgroup $C$ of central automorphisms is normal, $\alpha_{1} \circ \beta_{2} \circ \alpha_{2} \circ \gamma$ is of the required form.

\section{Central endomorphisms, Central automorphisms and non-Abelian FACTORS OF IRREDUCIBLE COMPLEX REFLECTION GROUPS}

In this section, we describe the central factor and the group $C$ of central automorphisms for irreducible complex reflection groups.

We relate the problem, for complex reflection groups, to linear characters. We recall that the group of linear characters, or equivalently the abelianization of $G$, is isomorphic to $\prod_{H} \mathbb{Z} / \# C_{G}(H)$ where $H$ runs over representatives of hyperplane orbits. If $S$ is the generating subset we have taken for $G$, the hyperplane orbits define a partition $\sigma$ of $S$, and a linear character $\chi$ is specified by specifying arbitrarily $\chi(s) \in \mu_{e_{s}}$ for one $s$ in each part of $\sigma$, where $e_{s}$ is the order of $s$.

Such a character $\chi$ will give rise to a central endomorphism if $\chi(s) \in \mu_{\# Z G}$ for any $s \in S$. Let $z$ be a generator of $Z G ; \alpha_{\chi}$ will be an automorphism if and only if $z \chi(z)^{-1}$ is still a generator of $Z G$. Notice that $\operatorname{ker} \alpha_{\chi} \subseteq Z G$.

We now proceed, case-by-case, to describe $\sigma$ and give an expression for $z$ in terms of the generators $S$, from which we will deduce $C$ and the central factor of $G$ as well as a possible non-abelian factor. 
For the groups $G(d e, e, r)$, we have $S=\left\{t^{\prime}, s_{1}^{\prime}, s_{1}, \ldots, s_{r-1}\right\}$ (where $t^{\prime}=t^{e}$ and $s_{1}^{\prime}=s_{1}^{t}$ as in section 2.1) where $t^{\prime}$ is omitted if $d=1$ and $s_{1}^{\prime}$ is omitted if $e=1$. Expressions for a generator of $Z G$ can be found in [BMR]; it is equal to $z=\left(t^{\prime} s_{1} \ldots s_{r-1}\right)^{r}$ if $e=1$, and $z=t^{\prime r / \operatorname{gcd}(e, r)}\left(s_{1}^{\prime} s_{1} \ldots s_{r-1}\right)^{\frac{e(r-1)}{\operatorname{gcd}(e, r)}}$ in the general case (this expression is still valid for $d=1$ by setting $t^{\prime}=1$ in that case). We have $Z G \simeq \mu_{d \cdot \operatorname{gcd}(e, r)}$.

When writing the partition $\sigma$, we decorate the parts where $e_{s} \neq 2$ with the value of $e_{s}$, separated by a semicolon. Thus $\sigma$ is $\left\{t^{\prime} ; d\right\},\left\{s_{1}^{\prime}, s_{1}, \ldots, s_{r-1}\right\}$ except when $r=2$ and $e$ is even, in which case it is $\left\{t^{\prime} ; d\right\},\left\{s_{1}^{\prime}\right\},\left\{s_{1}\right\}$. We find that a character $\chi$ gives rise to a central endomorphism of $G(d e, e, r)$ if and only if $\chi\left(s_{1}^{\prime}\right)=\chi\left(s_{i}\right)=1$ when $d \operatorname{gcd}(e, r)$ is odd. We assume this condition in the sequel.

We first consider the case where $r>2$ or $r=2$ and $e$ is odd. Then $\chi(z)=$ $\chi\left(t^{\prime}\right)^{\frac{r}{\operatorname{gcd}(e, r)}} \chi\left(s_{1}\right)^{\frac{e}{\operatorname{gcd}(e, r)} r(r-1)}=\chi\left(t^{\prime}\right)^{\frac{r}{\operatorname{gcd}(e, r)}}$. If we define $j$ by $\chi\left(t^{\prime}\right)=\zeta_{d}^{j}$ and $0 \leq$ $j<d$, and if $\alpha_{\chi}$ is the corresponding central endomorphism, we then have that

$$
\begin{aligned}
\# \operatorname{ker}\left(\alpha_{\chi}\right)= & \#\left\{i \in\{0, \ldots, d \operatorname{gcd}(e, r)-1\} \mid \chi\left(t^{\prime}\right)^{\frac{r i}{\operatorname{gcd}(e, r)}}=\zeta_{d \operatorname{gcd}(e, r)}^{i}\right\} \\
& =\#\{i \in\{0, \ldots, d \operatorname{gcd}(e, r)-1\} \mid i(r j-1) \equiv 0 \quad(\bmod d \operatorname{gcd}(e, r))\} .
\end{aligned}
$$

We define $d_{r^{\prime}}$ to be the largest factor of $d$ which is prime to $r$; notice that $r j-1$ and $d \operatorname{gcd}(e, r)$ being coprime is equivalent to $r j-1$ and $d_{r^{\prime}}$ being coprime.

We thus find that:

- $\alpha_{\chi}$ is an automorphism if and only if $r j-1$ is prime to $d_{r^{\prime}}$.

- The maximal kernel for an $\alpha_{\chi}$ is of cardinality $d_{r^{\prime}}$, obtained when $r j \equiv 1$ $\left(\bmod d_{r^{\prime}}\right)$. There always exists such a $\chi$ as $d_{r^{\prime}}$ and $r$ are coprime.

If $r j \equiv 1\left(\bmod d_{r^{\prime}}\right)$ we have $\operatorname{det}\left(\alpha_{\chi}\left(t^{\prime}\right)\right)=\operatorname{det}\left(t^{\prime}\right) / \chi\left(t^{\prime}\right)^{r}=\zeta_{d}^{1-r j} \in \mu_{d / d_{r^{\prime}}}$, thus $\operatorname{Im}\left(\alpha_{\chi}\right) \subseteq G\left(d e, d_{r^{\prime}} e, r\right)$ unless it is possible to take $\chi\left(s_{i}\right)=-1$ and $-1 \notin$ $G\left(d e, d_{r^{\prime}} e, r\right)$. For this to happen, we need $\# Z G\left(d e, d_{r^{\prime}} e, r\right)=\frac{d}{d_{r^{\prime}}} \operatorname{gcd}\left(d_{r^{\prime}} e, r\right)=$ $\frac{d}{d_{r^{\prime}}} \operatorname{gcd}(e, r)$ to be odd; but since we have the condition $\chi\left(s_{i}\right)=1$ if $d \operatorname{gcd}(e, r)$ is odd, $\chi\left(s_{i}\right)=-1$ implies that $d \operatorname{gcd}(e, r)=d_{r^{\prime}} \frac{d}{d_{r^{\prime}}} \operatorname{gcd}(e, r)$ is even hence $d_{r^{\prime}}$ is even, so $r$ is odd because $d_{r^{\prime}}$ and $r$ are coprime, and $d$ is even because $d_{r^{\prime}}$ divides $d$. We finally get that when $r>2$ or $r=2$ and $e$ is odd the central factor of $G(d e, e, r)$ is $\mathbb{Z} / d_{r^{\prime}}$ and the non-abelian factor is unique equal to $G\left(d e, d_{r^{\prime}} e, r\right)$, unless $d$ is even and $r$ odd when there is another possibility for the non-abelian factor.

In the remaining case of $G\left(2 d e^{\prime}, 2 e^{\prime}, 2\right)$, the partition $\sigma$ is $\left\{t^{\prime} ; d\right\},\left\{s_{1}^{\prime}\right\},\left\{s_{1}\right\}$, and $z=t^{\prime}\left(s_{1}^{\prime} s_{1}\right)^{e^{\prime}}$ is of order $2 d$.

Define $\epsilon=0$ if $\chi\left(s_{1}^{\prime} s_{1}\right)=1$ or $e^{\prime}$ is even, and $\epsilon=d$ otherwise, and define $j$ by $\chi\left(t^{\prime}\right)=\zeta_{d}^{j}$. An analysis similar to the above case shows that \# $\operatorname{ker} \alpha_{\chi}=\#\{i \in$ $\{0, \ldots, 2 d-1\} \mid i(2 j+\epsilon-1) \equiv 0(\bmod 2 d)\}$.

We find that $\chi$ defines a central automorphism if and only if $\epsilon$ is even and $2 j+\epsilon-1$ is prime to $d_{2^{\prime}}$, where $d_{2^{\prime}}$ is the largest odd divisor of $d$.

The maximal kernel of $\alpha_{\chi}$ is of order $d_{2^{\prime}}$ obtained, e.g., for $\epsilon=0$ and $2 j \equiv 1$ $\left(\bmod d_{2^{\prime}}\right)$, except when $d$ and $e^{\prime}$ are both odd, in which case it is of order $2 d$, obtained when $\chi\left(s_{1}^{\prime} s_{1}\right)=-1$ and $j \equiv(1+d) / 2(\bmod d)$. It can be checked that in this last case there are two possibilities for the image of $\alpha_{\chi}$; if $\chi\left(s_{1}^{\prime}\right)=-1$ it lies in $G\left(d e^{\prime}, d e^{\prime}, 2\right)$, so we get 
Proposition 13.1. Decompositions of $G(d e, e, r)$ into a central factor and a nonabelian indecomposable factor are as follows:

- If $r=2, d$ is odd and $e=2 e^{\prime}$ with $e^{\prime}$ odd, a decomposition is $\mathbb{Z} / 2 d \times$ $G\left(d e^{\prime}, d e^{\prime}, 2\right)$; there is one other possibility for the non-abelian factor.

- In the other cases a decomposition is $\mathbb{Z} / d_{r^{\prime}} \times G\left(d e, d_{r^{\prime}} e, r\right)$; this decomposition is unique unless $d$ is even and $r$ odd, in which case there is one other possibility for the non-abelian factor.

Table 3 gives for exceptional groups the partition $\sigma$ (where each part is decorated by the order of the corresponding reflections, when this order is not 2), the order of $Z G$, an expression in terms of $S$ of a generator of $Z G$ (such expressions can be found in [BMR, table 4] and in [BM]), under the column "automorphism" conditions describing the linear characters giving rise to an element of $C$ (this column is empty when there are no conditions), and under the column "decomposition" the decomposition of $G$. In this table, $\mathfrak{A}_{5} .2$ denotes the (unique) non-split central extension of $\mathfrak{A}_{5}$ by $\mathbb{Z} / 2$, and $\mathrm{SO}_{5}\left(\mathbb{F}_{3}\right)^{\prime}$ is the commutator subgroup of $\mathrm{SO}_{5}\left(\mathbb{F}_{3}\right)$.

This decomposition turns out to be unique in each case, except for $G_{5}$ and $G_{7}$ where the non-abelian factor given is one of 3 possibilities.

Table 3: Central endomorphisms

\begin{tabular}{|c|c|c|c|c|c|}
\hline$G$ & $\sigma$ & $\# Z G$ & $z$ & automorphism & decomposition \\
\hline$G_{4}$ & $\{s, t ; 3\}$ & 2 & $(s t)^{3}$ & $\chi(s)=1$ & $G_{4}$ \\
\hline$G_{5}$ & $\{s ; 3\},\{t ; 3\}$ & 6 & $(s t)^{2}$ & $\chi(s t) \neq \zeta_{3}$ & $\mathbb{Z} / 3 \times G_{4}$ \\
\hline$G_{6}$ & $\{s\},\{t ; 3\}$ & 4 & $(s t)^{3}$ & $\chi(t)=1$ & $G_{6}$ \\
\hline$G_{7}$ & $\{s\},\{t ; 3\},\{u ; 3\}$ & 12 & stu & $\chi(t u) \neq \zeta_{3}$ & $\mathbb{Z} / 3 \times G_{6}$ \\
\hline$G_{8}$ & $\{s, t ; 4\}$ & 4 & $(s t)^{3}$ & & $G_{8}$ \\
\hline$G_{9}$ & $\{s\},\{t ; 4\}$ & 8 & $(s t)^{3}$ & & $G_{9}$ \\
\hline$G_{10}$ & $\{s ; 3\},\{t ; 4\}$ & 12 & $(s t)^{2}$ & $\chi(s) \neq \zeta_{3}$ & $\mathbb{Z} / 3 \times G_{8}$ \\
\hline$G_{11}$ & $\{s\},\{t ; 3\},\{u ; 4\}$ & 24 & stu & $\chi(t) \neq \zeta_{3}$ & $\mathbb{Z} / 3 \times G_{9}$ \\
\hline$G_{12}$ & $\{s, t, u\}$ & 2 & $(s t u)^{4}$ & & $G_{12}$ \\
\hline$G_{13}$ & $\{s\},\{t, u\}$ & 4 & $(s t u)^{3}$ & & $G_{13}$ \\
\hline$G_{14}$ & $\{s\},\{t ; 3\}$ & 6 & $(s t)^{4}$ & $\chi(t) \neq \zeta_{3}$ & $\mathbb{Z} / 3 \times G_{12}$ \\
\hline$G_{15}$ & $\{s\},\{t\},\{u ; 3\}$ & 12 & $u(s t)^{2}$ & $\chi(t) \neq \zeta_{3}$ & $\mathbb{Z} / 3 \times G_{13}$ \\
\hline$G_{16}$ & $\{s, t ; 5\}$ & 10 & $(s t)^{3}$ & $\chi(s) \neq \zeta_{5}^{2}$ & $\mathbb{Z} / 5 \times\left(\mathfrak{A}_{5} .2\right)$ \\
\hline$G_{17}$ & $\{s\},\{t ; 5\}$ & 20 & $(s t)^{3}$ & $\chi(t) \neq \zeta_{5}^{2}$ & $\mathbb{Z} / 5 \times G_{22}$ \\
\hline$G_{18}$ & $\{s ; 3\},\{t ; 5\}$ & 30 & $(s t)^{2}$ & $\chi(s) \neq \zeta_{3}, \chi(t) \neq \zeta_{5}^{2}$ & $\mathbb{Z} / 15 \times\left(\mathfrak{A}_{5} .2\right)$ \\
\hline$G_{19}$ & $\{s\},\{t ; 3\},\{u ; 5\}$ & 60 & stu & $\chi(t) \neq \zeta_{3}, \chi(u) \neq \zeta_{5}^{2}$ & $\mathbb{Z} / 15 \times G_{22}$ \\
\hline$G_{20}$ & $\{s, t ; 3\}$ & 6 & $(s t)^{5}$ & $\chi(s) \neq \zeta_{3}$ & $\mathbb{Z} / 3 \times\left(\mathfrak{A}_{5} \cdot 2\right)$ \\
\hline$G_{21}$ & $\{s\},\{t ; 3\}$ & 12 & $(s t)^{5}$ & $\chi(t) \neq \zeta_{3}$ & $\mathbb{Z} / 3 \times G_{22}$ \\
\hline$G_{22}$ & $\{s, t, u\}$ & 4 & $(s t u)^{5}$ & & $G_{22}$ \\
\hline$G_{23}$ & $\{s, t, u\}$ & 2 & $(s t u)^{5}$ & $\chi(s)=1$ & $\mathbb{Z} / 2 \times \mathfrak{A}_{5}$ \\
\hline$G_{24}$ & $\{s, t, u\}$ & 2 & $(s t u)^{7}$ & $\chi(s)=1$ & $\mathbb{Z} / 2 \times \mathrm{GL}_{3}\left(\mathbb{F}_{2}\right)$ \\
\hline$G_{25}$ & $\{s, t, u ; 3\}$ & 3 & $(s t u)^{4}$ & & $G_{25}$ \\
\hline$G_{26}$ & $\{s\},\{t, u ; 3\}$ & 6 & $(s t u)^{3}$ & $\chi(s)=1$ & $\mathbb{Z} / 2 \times G_{25}$ \\
\hline$G_{27}$ & $\{s, t, u\}$ & 6 & $(u t s)^{5}$ & $\chi(s)=1$ & $\mathbb{Z} / 2 \times \mathfrak{A}_{6}$ \\
\hline$G_{28}$ & $\{s, t\},\{u, v\}$ & 2 & $(s t u v)^{6}$ & & $G_{28}$ \\
\hline$G_{29}$ & $\{s, t, u, v\}$ & 4 & $(s t v u)^{5}$ & & $G_{29}$ \\
\hline$G_{30}$ & $\{s, t, u, v\}$ & 2 & $(s t u v)^{15}$ & & $G_{30}$ \\
\hline
\end{tabular}




\begin{tabular}{|l|ccccc|}
\hline$G$ & $\sigma$ & $\# Z G$ & $z$ & automorphism & decomposition \\
\hline$G_{31}$ & $\{s, t, u, v, w\}$ & 4 & $(\text { stuvw })^{6}$ & & $G_{31}$ \\
$G_{32}$ & $\{s, t, u, v ; 3\}$ & 6 & $(s t u v)^{5}$ & $\chi(s) \neq \zeta_{3}^{2}$ & $\mathbb{Z} / 3 \times \mathrm{Sp}_{4}\left(\mathbb{F}_{3}\right)$ \\
$G_{33}$ & $\{s, t, u, v, w\}$ & 2 & $(\text { stvwu })^{9}$ & $\chi(s)=1$ & $\mathbb{Z} / 2 \times \mathrm{SO}_{5}\left(\mathbb{F}_{3}\right)^{\prime}$ \\
$G_{34}$ & $\{s, t, u, v, w, x\}$ & 6 & $(\text { stvwux })^{7}$ & & $G_{34}$ \\
$G_{35}$ & $\left\{s_{1}, \ldots, s_{6}\right\}$ & 1 & 1 & $\chi(s)=1$ & $G_{35}$ \\
$G_{36}$ & $\left\{s_{1}, \ldots, s_{7}\right\}$ & 2 & $\left(s_{1} \ldots s_{7}\right)^{9}$ & $\chi(s)=1$ & $\mathbb{Z} / 2 \times \mathrm{SO}_{7}\left(\mathbb{F}_{2}\right)$ \\
$G_{37}$ & $\left\{s_{1}, \ldots, s_{8}\right\}$ & 2 & $\left(s_{1} \ldots s_{8}\right)^{15}$ & & $G_{37}$ \\
\hline
\end{tabular}

We remark that for any irreducible complex reflection group, the non-abelian factor of $G$ can always be taken to be a reflection subgroup, unless $G$ is an exceptional group in the list $G_{16}, G_{18}, G_{20}, G_{23}, G_{24}, G_{27}, G_{32}, G_{33}, G_{36}$.

The information we give is sufficient, in rank 2 , to determine the intersection of $C$ with the automorphisms which preserve the reflections, thus determining the structure of $\operatorname{Aut}(G)$ in that case. For exceptional groups, the isomorphism type of $C$ is given in Table 1 of $\mathrm{Bes}$ (we note on this table that the group structure of $C$ given by composition of automorphisms is unrelated to the group structure on $\operatorname{Hom}\left(G, \mathbb{C}^{\times}\right)$; in particular, $C$ may be non-commutative).

Assume that $G$ is of rank 2 and let $H$ be the group of Galois automorphisms, which is well-determined by Theorem 1.6 since $\operatorname{gcd}(e, r) \leq 2$ in our case. It follows from the above table that $C=H$ for $G_{12}, G_{13}, G_{14}, G_{15}$, that $H$ is of index 2 in $C$ for $G_{8}, G_{9}, G_{10}, G_{11}$, that $C$ is of index 2 in $H$ for $G_{4}, G_{6}, G_{20}, G_{20}, G_{22}$, and that $C \cap H$ is of index 2 in both $C$ and $H$ for $G_{16}, G_{17}, G_{18}, G_{19}$. Finally, in the two cases $G_{5}$ and $G_{7}$ where $N$ is not trivial, we have $N \subset C$. In these cases $C$ is non-commutative, isomorphic to $\mathfrak{S}_{3}$ for $G_{5}$ and $\mathbb{Z} / 2 \times \mathfrak{S}_{3}$ for $G_{7}$. In both cases $C \cap H$ is the center of $C$, which is trivial for $G_{5}$ and equal to $\mathbb{Z} / 2$ for $G_{7}$.

\section{ACKNOWLEDGEMENTS}

The authors thank David Harari for teaching them about the Brauer group, Gunter Malle and the referee for a careful reading of various versions of the paper, and Luis Paris for suggesting the use of the Krull-Remak-Schmidt theorem.

\section{REFERENCES}

[Ar] S. Ariki, "Representation theory of a Hecke algebra for $G(r, p, n)$ ", J. Algebra 177 (1995), 164-185. MR1356366 (96j:20021)

[ArKo] S. Ariki and K. Koike, "A Hecke algebra of $\mathbb{Z} / r \mathbb{Z} 2 \mathfrak{S}_{n}$ and construction of its irreducible representations", Adv. Math. 106 (1994), 216-243. MR.1279219 (95h:20006)

[Bena] M. Benard, "Schur indices and splitting fields of the Unitary reflection groups" J. Algebra 38 (1976), 318-342. MR0401901 (53:5727)

[Bens] D. Benson "Polynomial invariants of finite groups", LMS Lecture Note Series, 190. Cambridge University Press, Cambridge, 1993. MR 1249931 (94j:13003)

[Bes] D. Bessis, "Sur le corps de définition d'un groupe de réflexions complexe", Comm. Algebra 25 (1997), 2703-2716. MR.1459587 (98g:20017)

[Be2] D. Bessis, "Zariski theorems and diagrams for braid groups", Invent. Math. 145 (2001), 487-507. MR 1856398 (2002g:20066)

[BM] D. Bessis, J. Michel, "Explicit presentations for exceptional braid groups", Experimental Mathematics 13 (2004), 257-266. MR2103323 (2006b:20051)

[Bbk1] N. Bourbaki, "Algèbre", Chap. V, "Corps commutatifs", Hermann, Paris, 1950.

[Bbk2] N. Bourbaki, "Groupes et algèbres de Lie", Chap. V, "Groupes engendrés par des réflexions", Hermann, Paris, 1968. MR0240238 (39:1590) 
[BMM] M. Broué, G. Malle and J. Michel, "Towards Spetses I", Transformation Groups 4 (1999), 157-218. MR1712862 (2001b:20082)

[BMR] M. Broué, G. Malle and R. Rouquier, "Complex reflection groups, braid groups, Hecke algebras", J. Reine Angew. Math. 500 (1998), 127-190. MR1637497 (99m:20088)

[CHEVIE] See www.math.jussieu.fr/ jmichel/chevie.

[Cohen] A. Cohen, "Finite complex reflection groups", Annales de l'E.N.S. 9 (1976), 379-436. MR.0422448 (54:10437)

[FH] W. Fulton and J. Harris, "Representation theory", Springer G.T.M. 129 (1991). MR.1153249 (93a:20069)

[M1] I. Marin, "Sur les représentations de Krammer génériques", Ann. Inst. Fourier 57 (2007) 1883-1925. MR2377890 (2009d:20083)

[M2] I. Marin, "Branching properties for the groups $G(d e, e, r)$ ", J. Algebra 323 (2010), 966-982.

[OT] P. Orlik and H. Terao, "Arrangements of hyperplanes", Springer G.M.W. 300 (1991). MR.1217488 (94e:52014)

[R] E. W. Read, "On the finite imprimitive unitary reflection groups", J. Algebra 45(1977), 439-452. MR0442074 (56:462)

[Ro] D. Robinson, "A course in the theory of groups", G.T.M. 80 (1982), Springer-Verlag. MR648604 (84k:20001)

[Se] J.-P. Serre, "Corps locaux", Hermann, Paris, 1968. MR 0354618 (50:7096)

[Ze] A. V. Zelevinsky "Representations of finite classical groups - a Hopf algebra approach", Springer SLN 869 (1981). MR643482(83k:20017)

Institut de Mathématiques de Jussieu, Université Paris VII, 175, Rue du Chevaleret, 75013 PARIS

E-mail address: marin@math.jussieu.fr

Institut de Mathématiques de Jussieu, Université Paris ViI, 175, Rue du Chevaleret, 75013 PARIS

E-mail address: jmichel@math.jussieu.fr 